\title{
F-SINGULARITIES VIA ALTERATIONS
}

\author{
MANUEL BLICKLE, KARL SCHWEDE, KEVIN TUCKER
}

\begin{abstract}
We give characterizations of test ideals and $F$-rational singularities via (regular) alterations. Formally, the descriptions are analogous to standard characterizations of multiplier ideals and rational singularities in characteristic zero via log resolutions. Lastly, we establish Nadel-type vanishing theorems (up to finite maps) for test ideals, and further demonstrate how these vanishing theorems may be used to extend sections.
\end{abstract}

\section{INTRODUCTION}

In this paper we define an ideal, in arbitrary equal characteristic, which coincides with the multiplier ideal over $\mathbb{C}$, and coincides with the test ideal in characteristic $p>0$. This justifies the maxim:

The test ideal and multiplier ideal are morally equivalent.

We state our main theorem.

Main Theorem (Theorem 4.6. Corollary 4.8, Theorem 8.2). Suppose that $X$ is a normal algebraic variety over a perfect field and $\Delta$ is a $\mathbb{Q}$-divisor on $X$ such that $K_{X}+\Delta$ is $\mathbb{Q}$-Cartier. Consider the ideal

$$
J:=\bigcap_{\pi: Y \longrightarrow X} \text { Image }\left(\pi_{*} \mathscr{O}_{Y}\left(\left\lceil K_{Y}-\pi^{*}\left(K_{X}+\Delta\right)\right\rceil\right) \stackrel{\operatorname{Tr}_{\pi}}{\longrightarrow} K(X)\right) .
$$

Here the intersection runs over all generically finite proper separable maps $\pi: Y \rightarrow X$ where $Y$ is regular (or equivalently just normal), and the map to the function field $K(X)$ is induced by the Grothendieck trace map $\operatorname{Tr}_{\pi}: \pi_{*} \omega_{Y} \rightarrow \omega_{X}$ (if $K_{Y}=\pi^{*} K_{X}+\operatorname{Ram}_{\pi}$ over the locus where $\pi$ is finite, then $\operatorname{Tr}_{\pi}: \pi_{*} \mathscr{O}_{Y}\left(K_{Y}\right) \rightarrow \mathscr{O}_{X}\left(K_{X}\right)$ is induced by the field trace $\left.\operatorname{Tr}_{K(Y) / K(X)}: K(Y) \rightarrow K(X)\right)$. We obtain the following:

(a) If $X$ is of equal characteristic zero, then $J=\mathcal{J}(X ; \Delta)$, the multiplier ideal of $(X, \Delta)$.

(b) If $X$ is of equal characteristic $p>0$, then $J=\tau(X ; \Delta)$, the test ideal of $(X, \Delta)$.

Furthermore, in either case, the intersection defining $J$ stabilizes: in other words, there is always a generically finite separable proper map $\pi: Y \rightarrow X$ with $Y$ regular such that $J=\operatorname{Image}\left(\pi_{*} \mathscr{O}_{Y}\left(K_{Y}-\pi^{*}\left(K_{X}+\Delta\right)\right) \stackrel{\operatorname{Tr}_{\pi}}{\longrightarrow} K(X)\right)$.

In fact, we prove a number of variants on the above theorem in further generality, i.e. for various schemes other than varieties over a perfect field.

2010 Mathematics Subject Classification. 14F18, 13A35, 14F17, 14B05, 14E15.

Key words and phrases. Test ideal, multiplier ideal, alteration, rational singularities, $F$-rational singularities, Nadel vanishing.

The first author was partially supported by a DFG Heisenberg Fellowship and the DFG research grant SFB/TRR45.

The second author was partially supported by an NSF Postdoctoral Fellowship \#0703505, the NSF grant \#1064485, the NSF FRG grant \#1265261, the NSF CAREER grant \#1252860 and a Sloan Fellowship.

The third author was partially supported by an NSF Postdoctoral fellowship \#1004344 and NSF grant \#1303077. 
Of course, there are two different statements here. In characteristic zero, this statement can be viewed as a generalization of the transformation rule for multiplier ideals under generically finite proper dominant maps, see [Laz04, Theorem 9.5.42] or [Ein97, Proposition 2.8]. In positive characteristic, a basic case of the theorem is the following characterization of $F$-rational singularities - which is interesting in its own right. Recall that an alteration is a proper and generically finite map $\pi: Y \rightarrow X$, it is called a regular alteration if $Y$ is a regular scheme dJ96.

Corollary Corollary 3.6, cf. [HL07, HY11]). An F-finite ring $R$ of characteristic $p>0$ is $F$-rational if and only if it is Cohen-Macaulay and for every alteration (equivalently every regular separable alteration if $R$ is of finite type over a perfect field, equivalently every finite dominant map with $Y$ normal) $f: Y \rightarrow \operatorname{Spec} R$, the map $f_{*} \omega_{Y} \rightarrow \omega_{R}$ is surjective.

The proof of this special case is in fact the key step in the proof of the main theorem in positive characteristic. The central ingredients in its proof are the argument of K. Smith Smi97b that $F$-rational singularities are pseudo-rational, and the work of C. Huneke and G. Lyubeznik on annihilating local cohomology using finite covers HL07, (cf. HH92, HY11, SS12]). The proof of the Main Theorem additionally utilizes transformation rules for test ideals under finite morphisms [ST10].

In this paper, we also give a transformation rule for test ideals under proper dominant (and in particular proper birational) maps between varieties of the same dimension. More precisely, for any normal (but not necessarily proper) variety $Y$ and $\mathbb{Q}$-divisor $\Gamma$, we define a canonical submodule $T^{0}(Y, \Gamma) \subseteq H^{0}\left(Y, \mathscr{O}_{Y}\left(\left\lceil K_{Y}+\Gamma\right\rceil\right)\right)$. We use this submodule to obtain a transformation rule for test ideals under alterations.

Theorem 1 (Theorem 6.8). Suppose that $\pi: Y \rightarrow X=\operatorname{Spec} R$ is a proper dominant generically finite map of normal varieties over a perfect (or even $F$-finite) field of characteristic $p>0$. Further suppose that $\Delta$ is a $\mathbb{Q}$-divisor on $X$ such that $K_{X}+\Delta$ is $\mathbb{Q}$-Cartier.

Consider the canonically determined submodule (see Definition 6.1)

$$
\left.T^{0}\left(Y,-\pi^{*}\left(K_{X}+\Delta\right)\right) \subseteq H^{0}\left(Y, \mathscr{O}_{Y}\left(\left\lceil K_{Y}-\pi^{*}\left(K_{X}+\Delta\right)\right)\right\rceil\right)\right)
$$

of sections which are in the image of the trace map for any alteration of $Y$. Then the global sections of $\tau(X ; \Delta)$ coincide with the image of $T^{0}\left(Y,-\pi^{*}\left(K_{X}+\Delta\right)\right)$ under the map

$$
H^{0}\left(Y, \mathscr{O}_{Y}\left(\left\lceil K_{Y}-\pi^{*}\left(K_{X}+\Delta\right)\right\rceil\right)\right) \stackrel{\operatorname{Tr}_{\pi}}{\longrightarrow} K(X)
$$

which is induced by the trace $\operatorname{Tr}_{\pi}: \pi_{*} \omega_{Y} \rightarrow \omega_{X}$.

We also prove a related transformation rule for multiplier ideals under arbitrary proper dominant maps in Theorem 8.3 .

Perhaps the most sorely missed tools in positive characteristic birational algebraic geometry (in comparison to characteristic zero) are vanishing theorems for cohomology. Indeed, Kodaira vanishing fails in positive characteristic Ray78. However, if $X$ is projective in characteristic $p>0$ and $\mathscr{L}$ is a "positive" line-bundle, cohomology classes $z \in H^{i}\left(X, \mathscr{L}^{-1}\right)$ can often be killed by considering their images in $H^{i}\left(Y, f^{*} \mathscr{L}^{-1}\right)$ for finite covers $f: Y \rightarrow X$. For example, if $i \geq 0$ and $\mathscr{L}$ big and semi-ample, it was shown in Bha12, Bha10 that there exists such a cover killing any cohomology class $\eta \in H^{i}\left(X, \mathscr{L}^{-1}\right)$ for $i<\operatorname{dim} X(c f$. [HH92, Smi97c, Smi97a]). When we combine our main result with results from Bha10, we obtain the following variant of a Nadel-type vanishing theorem in characteristic $p>0$ (and a relative version). Notably, we need not require a W2 lifting hypothesis.

Theorem 2 (Theorem 5.5) . Let $X$ be a normal proper algebraic variety of finite type over a perfect (or even F-finite) field of characteristic $p>0, L$ a Cartier divisor, and $\Delta \geq 0$ 
$a \mathbb{Q}$-divisor such that $K_{X}+\Delta$ is $\mathbb{Q}$-Cartier. Suppose that $L-\left(K_{X}+\Delta\right)$ is a big and semi-ample $\mathbb{Q}$-divisor. Then there exists a finite surjective map $f: Y \rightarrow X$ such that:

(a) The natural map $f_{*} \mathscr{O}_{Y}\left(\left\lceil K_{Y}+f^{*}\left(L-K_{X}-\Delta\right)\right\rceil\right) \rightarrow \mathscr{O}_{X}(L)$, induced by the trace map, has image $\tau(X ; \Delta) \otimes \mathscr{O}_{X}(L)$.

(b) The induced map on cohomology

$$
H^{i}\left(Y, \mathscr{O}_{Y}\left(\left\lceil K_{Y}+f^{*}\left(L-K_{X}-\Delta\right)\right\rceil\right)\right) \rightarrow H^{i}\left(X, \tau(X ; \Delta) \otimes \mathscr{O}_{X}(L)\right)
$$

is zero for all $i>0$.

Applying the vanishing theorem above, we obtain the following extension result.

Theorem 3 (Theorem [7.6). Let $X$ be a normal algebraic variety which is proper over a perfect (or even $F$-finite) field of characteristic $p>0$ and $D$ is a Cartier divisor on $X$. Suppose that $\Delta$ is a $\mathbb{Q}$-divisor having no components in common with $D$ and such that $K_{X}+\Delta$ is $\mathbb{Q}$-Cartier. Further suppose that $L$ is a Cartier divisor on $X$ such that $L-\left(K_{X}+D+\Delta\right)$ is big and semi-ample. Consider the natural restriction map

$$
\gamma: H^{0}\left(X, \mathscr{O}_{X}(\lceil L-\Delta\rceil) \rightarrow H^{0}\left(D, \mathscr{O}_{D}\left(\left.L\right|_{D}-\left.\lfloor\Delta\rfloor\right|_{D}\right)\right.\right.
$$

Then

$$
T^{0}\left(D,\left.L\right|_{D}-\left(K_{D}+\left.\Delta\right|_{D}\right)\right) \subseteq \gamma\left(T^{0}\left(X, D+L-\left(K_{X}+D+\Delta\right)\right)\right)
$$

noting that $T^{0}\left(D,\left.L\right|_{D}-\left(K_{D}+\left.\Delta\right|_{D}\right)\right) \subseteq H^{0}\left(D, \mathscr{O}_{D}\left(\left\lceil\left. L\right|_{D}-\left.\lfloor\Delta\rfloor\right|_{D}\right)\right\rceil\right)$. In particular, if $T^{0}\left(D,\left.L\right|_{D}-\left(K_{D}+\left.\Delta\right|_{D}\right)\right) \neq 0$, then $H^{0}\left(X, \mathscr{O}_{X}(\lceil L-\Delta\rceil) \neq 0\right.$.

Finally, let us remark that many of the results contained herein can be extended to excellent (but not necessarily $F$-finite) local rings with dualizing complexes; in fact, this is the setting of C. Huneke and G. Lyubeznik in [HL07. However, moving beyond the local case is then difficult essentially because we do not know the existence of test elements. For this reason, and also because our inspiration comes largely from (projective) geometry, we restrict ourselves to the $F$-finite setting throughout (note that any scheme of finite type over a perfect field is automatically $F$-finite).

Acknowledgements: The authors would like to thank Bhargav Bhatt, Christopher Hacon, Mircea Mustață and Karen Smith for valuable conversations. The authors would also like to thank all the referees for many very useful comments. Finally, the authors worked on this paper while visiting the Johannes Gutenberg-Universität Mainz during the summers of 2010 and 2011. These visits were funded by the SFB/TRR45 Periods, moduli, and the arithmetic of algebraic varieties.

\section{The trace MAP, MUltiplier IDEALS AND TEST IDEALS}

Multiplier ideals and test ideals are prominent tools in the study of singularities of algebraic varieties. Later in this section, we will briefly review their constructions together with those of certain variants - the multiplier and test module, respectively - related to various notions of rational singularities. In doing so, we emphasize a viewpoint that relies heavily on the use of the trace map of Grothendieck-Serre duality. In fact, the whole paper (particularly Sections 5, 6, and 7) relies on some of the more subtle properties of this theory. First however we give a brief introduction to this theory necessary to understand the main results of this paper that does not rely on any of these more subtle aspects. 
2.1. Maps derived from the trace map. This section is designed to be a friendly and brief introduction to the trace map at the level we will apply it for our main theorem. Therefore, in this subsection we only deal algebraic varieties of finite type over a perfect field (although everything can be immediately generalized to $F$-finite integral schemes). More general versions will be discussed later in Section 2.3. We will assume that the reader is familiar with canonical and dualizing modules at the level of [KM98, Section 5.5] and Har77, Chapter III, Section 7].

Suppose that $\pi: Y \rightarrow X$ is a proper generically finite map of varieties of finite type over a field $k$. A key tool in this paper is a trace map

$$
\operatorname{Tr}_{\pi}: \pi_{*} \omega_{Y} \rightarrow \omega_{X}
$$

Here $\omega_{Y}$ and $\omega_{X}$ denote suitable canonical modules on $Y$ and $X$ (which we assume exist). We will explain the origin of this map explicitly. Since any generically finite map can be factored into a composition of a finite and proper birational map, it suffices to deal with these cases separately:

Example 2.1 (Trace for proper birational morphism). Suppose that $\pi: Y \rightarrow X$ is a proper birational map between normal varieties. In this case, the trace map $\operatorname{Tr}_{\pi}: \pi_{*} \omega_{Y} \rightarrow \omega_{X}$ can be described in the following manner. Fix a canonical divisor $K_{Y}$ on $Y$ and set $K_{X}=$ $\pi_{*} K_{Y}$ (in other words, recall by definition that $\omega_{Y} \cong \mathscr{O}_{X}\left(K_{Y}\right)$ and requiring that $\pi_{*} K_{Y}=$ $K_{X}$ simply means that $K_{X}$ is the divisor on $X$ that agrees with $K_{Y}$ wherever $\pi$ is an isomorphism). Then $\pi_{*} \mathscr{O}_{Y}\left(K_{Y}\right)$ is a torsion-free sheaf whose reflexification is just $\mathscr{O}_{X}\left(K_{X}\right)$, since $\pi$ is an isomorphism outside a codimension 2 set of $X$. The trace map is simply the natural (reflexification) map $\pi_{*} \mathscr{O}_{Y}\left(K_{Y}\right) \hookrightarrow \mathscr{O}_{X}\left(K_{X}\right)$.

Example 2.2 (Trace for finite morphism). Suppose that $\pi: Y \rightarrow X$ is a finite surjective map of varieties. The trace map $\operatorname{Tr}_{\pi}: \pi_{*} \omega_{Y} \rightarrow \omega_{X}$ is then identified with the evaluation-at-1 map, $\pi_{*} \omega_{Y}:=\mathscr{H}_{0} m_{\mathscr{O}_{X}}\left(\pi_{*} \mathscr{O}_{Y}, \omega_{X}\right) \rightarrow \omega_{X}$ (the neophyte reader should take on faith that $\pi_{*} \omega_{Y} \cong \mathscr{H}_{o m_{\mathscr{O}_{X}}}\left(\pi_{*} \mathscr{O}_{Y}, \omega_{X}\right)$, or see Section 2.3 and Remark 2.17 for additional discussion). Assuming additionally that $\pi: Y \rightarrow X$ is a finite separable map of normal varieties with ramification divisor $\operatorname{Ram}_{\pi}$, we fix a canonical divisor $K_{X}$ on $X$ and set $K_{Y}=\pi^{*} K_{X}+\operatorname{Ram}_{\pi}$. Then the field-trace map

$$
\operatorname{Tr}_{K(Y) / K(X)}: K(Y) \rightarrow K(X)
$$

restricts to a map $\pi_{*} \mathscr{O}_{Y}\left(K_{Y}\right) \rightarrow \mathscr{O}_{X}\left(K_{X}\right)$ which can be identified with the Grothendieck trace map ( $c f$. ST10]).

Below in subsection 2.3 we will explain that this construction of a trace map is just an instance of a much more general theory contained in Grothendieck-Serre duality. We do not need this generality for our main theorem however.

We now mention two key properties that we will use repeatedly in this basic context.

Lemma 2.3 (Compatibilities of the trace map). Suppose that $\pi: Y \rightarrow X$ is a proper generically finite dominant morphism between varieties (or integral schemes). Fix $\operatorname{Tr}_{\pi}$ : $\pi_{*} \omega_{Y} \longrightarrow \omega_{X}$ to be the trace map as above.

(a) If additionally, $\rho: Z \longrightarrow Y$ is another proper generically finite dominant morphism and $\operatorname{Tr}_{\rho}: \rho_{*} \omega_{Z} \rightarrow \omega_{Y}$ is the associated trace map, then $\operatorname{Tr}_{\pi} \circ\left(\pi_{*} \operatorname{Tr}_{\rho}\right)=\operatorname{Tr}_{\pi \circ \rho}$.

(b) Additionally, if $U \subseteq X$ is open and $W=\pi^{-1}(U)$, then $\operatorname{Tr}_{\left.\pi\right|_{W}}=\left.\operatorname{Tr}_{\pi}\right|_{U}$ (here $\operatorname{Tr}_{\pi}$ : $\pi_{*} \omega_{Y} \rightarrow \omega_{X}$ is a map of sheaves on $X$ and so can be restricted to an open set). In other words, the trace map is compatible with open immersions.

Proof. These properties follow directly from the definition given. 
Because much of our paper is devoted to studying singularities defined by Frobenius, utilizing Lemma 2.3 we specialize Example 2.2 to the case where $\pi$ is the Frobenius.

Example 2.4 (Trace of Frobenius). Suppose that $X$ is a variety of finite type over a perfect field of characteristic $p>0$. Then consider the absolute Frobenius map $F: X \rightarrow X$, this map is not a map of varieties over $k$, but it is still a map of schemes. Using the fact (cf. Example 2.15) that $\mathscr{H}_{0} m_{\mathscr{O}_{X}}\left(F_{*} \mathscr{O}_{X}, \omega_{X}\right) \cong F_{*} \omega_{X}$, and applying Example 2.2, we obtain the evaluation-at-1 trace map,

$$
\operatorname{Tr}_{F}: F_{*} \omega_{X} \rightarrow \omega_{X}
$$

Because of the importance of this map in what follows, we will use the notation $\Phi_{X}$ to denote $\operatorname{Tr}_{F}$. As an endomorphism of $X$ one can compose the Frobenius with itself and obtain the $e$-iterated Frobenius $F^{e}$. It follows from Lemma 2.3 (a) that $\operatorname{Tr}_{F e}$ then coincides with the composition of $\operatorname{Tr}_{F}$ with itself $e$-times (appropriately pushed forward). Because of this, we use $\Phi_{X}^{e}$ to denote $\operatorname{Tr}_{F}$.

Now we come to a compatibility statement for images of trace maps that will be absolutely crucial later in the paper. This is essentially the dual statement to a key observation from Smi97b. We will generalize this later in Proposition 2.21 and also in the proof of Proposition 4.2.

Proposition 2.5. If $\pi: Y \rightarrow X$ is a proper dominant generically finite map of varieties, then the image of the trace map

$$
J_{\pi}:=\operatorname{Tr}_{\pi}\left(\pi_{*} \omega_{Y}\right) \subseteq \omega_{X}
$$

satisfies $\Phi_{X}\left(F_{*} J_{\pi}\right) \subseteq J_{\pi}$.

Proof. Consider the commutative diagram:

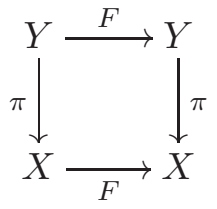

where the horizontal maps are the Frobenius on $X$ and $Y$ respectively. It follows from Lemma 2.3(a) that there is a commutative diagram

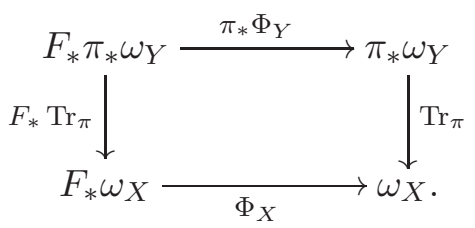

The claimed result follows immediately.

2.2. Pairs. The next step is to extend the trace map to incorporate divisors. Suppose that $X$ is a normal integral scheme. A $\mathbb{Q}$-divisor $\Gamma$ on $X$ is a formal linear combination of prime Weil divisors with coefficients in $\mathbb{Q}$. Writing $\Gamma=\sum b_{i} B_{i}$ where the $B_{i}$ are distinct prime divisors, we use $\lceil\Gamma\rceil=\sum\left\lceil b_{i}\right\rceil B_{i}$ and $\lfloor\Gamma\rfloor=\sum\left\lfloor b_{i}\right\rfloor B_{i}$ to denote the round up and round down of $\Gamma$, respectively. We say $\Gamma$ is $\mathbb{Q}$-Cartier if there exists an integer $n>0$ such that $n \Gamma$ is an integral (i.e. having integer coefficients) Cartier divisor, and the smallest such $n$ is called the index of $\Gamma$. An integral divisor $K_{X}$ with $\mathscr{O}_{X}\left(K_{X}\right) \cong \omega_{X}$ is called a canonical divisor. 
Definition 2.6. A pair $(X, \Delta)$ is the combined data of a normal integral scheme $X$ together with a $\mathbb{Q}$-divisor $\Delta$ on $X$. The pair $(X, \Delta)$ is called log- $\mathbb{Q}$-Gorenstein if $K_{X}+\Delta$ is $\mathbb{Q}$-Cartier.

We emphasize that log-QQQ-Gorenstein pairs need not be Cohen-Macaulay.

Convention 2.7. For $X$ normal and integral let $\Delta$ be a $\mathbb{Q}$-divisor on $X$. The choice of a rational section $s \in \omega_{X}$ gives a canonical divisor $K_{X}=K_{X, s}=\operatorname{div} s$ and also a map $\omega_{X} \subseteq$ $\omega_{X} \otimes K(X) \stackrel{s \mapsto 1}{\longrightarrow} K(X)$. Then the image of the inclusion $\omega_{X}\left(-K_{X, s}-\lfloor\Delta\rfloor\right) \stackrel{s \mapsto 1}{\longrightarrow} K(X)$ is the subsheaf $\mathscr{O}_{X}(-\lfloor\Delta\rfloor) \subseteq K(X)$. Note the image is independent of the choice of $s$ but the inclusion maps for different sections may differ by multiplication with a unit of $\mathscr{O}_{X}$.

Hence, every $\mathscr{O}_{X}$-submodule of $\omega_{X}\left(-\left\lfloor K_{X}+\Delta\right\rfloor\right)$ corresponds uniquely to an $\mathscr{O}_{X}$-submodule of $\mathscr{O}_{X}\left(-\lfloor\Delta\rfloor\right.$ ) (or even $\subseteq \mathscr{O}_{X}$ when $\Delta$ is effective). As such, we have chosen to accept certain abuses of notation in order to identify such submodules. For example, we may write $\omega_{X}\left(-\left\lfloor K_{X}+\Delta\right\rfloor\right) \subseteq K(X)$ (or $\subseteq \mathscr{O}_{X}$ when $\Delta$ is effective); however, while it is canonical as a subset (and equals $\mathscr{O}_{X}(-\lfloor\Delta\rfloor)$ ), the actual inclusion map involves the choice of a section (and is well defined only up to a multiplication by a unit of $\mathscr{O}_{X}$ ).

We now state a result incorporating divisors into the trace map.

Proposition 2.8. Suppose that $\pi: Y \rightarrow X$ is a proper dominant generically finite morphism between normal varieties, and let $\Delta$ be a $\mathbb{Q}$-divisor on $X$ such that $K_{X}+\Delta$ is $\mathbb{Q}$-Cartier. Then the trace map of $\pi$ induces a non-zero map

$$
\operatorname{Tr}_{\pi}: \pi_{*} \omega_{Y}\left(-\left\lfloor\pi^{*}\left(K_{X}+\Delta\right)\right\rfloor\right) \rightarrow \omega_{X}\left(-\left\lfloor K_{X}+\Delta\right\rfloor\right) .
$$

Proof. This result is simple based upon Examples 2.1 and 2.2 and so we leave it to the reader to verify. We carefully prove a more general result in Propositions 2.13]2.18 below.

2.3. Duality and the trace map. This section restates the results of the previous section in the more general language of dualizing complexes. While these results will be important for generalizations of our main theorem and for some of the Kodaira-type vanishing theorems, they are not needed for the main result stated in the introduction. Therefore, we invite the reader to skip the next section and instead read ahead to Section 2.4.

From now on we assume that all schemes $X$ are Noetherian, excellent, separated and possess a dualizing complex $\omega_{X}^{\cdot}$. This is a relatively mild condition, since, for example, all Noetherian schemes that are of finite type over a local Gorenstein ring of finite Krull dimension have a dualizing complex [Har66, Chapter V, Section 10]. By definition, Har66, Chapter V, Section 2], a dualizing complex on $X$ is an object in $D_{\text {coh }}^{b}(X)$ which has finite injective dimension and such that the canonical map $\mathscr{O}_{X} \rightarrow \mathbf{R} \mathscr{H}_{0} m_{X}\left(\omega_{X}^{\bullet}, \omega_{X}^{\bullet}\right)$ is an isomorphism in $D_{\text {coh }}^{b}(X)$.

Since dualizing complexes are defined by properties in the derived category, they are only unique up to quasi-isomorphism. But even worse, if $\omega^{\bullet}$ is a dualizing complex, then so is $\omega^{\bullet} \otimes \mathscr{L}[n]$ for any integer $n$ and line-bundle $\mathscr{L}$. But this is all the ambiguity there is for a connected scheme: if $\Omega^{\bullet}$ is another dualizing complex then there is a unique line-bundle $\mathscr{L}$ and a unique shift $n$ such that $\Omega^{\bullet}$ is quasi-isomorphic to $\omega^{\bullet} \otimes \mathscr{L}[n]$, see Har66. Theorem V.3.1]. The ambiguity with respect to shift is the least serious. For this, we say that a dualizing complex on an integral scheme (or a local scheme) is said to be normalized if the first non-zero cohomology of $\omega_{X}^{\cdot}$ lies in degree $(-\operatorname{dim} X)$.

A canonical module $\omega_{X}$ on a reduced and connected scheme $X$ is a coherent $\mathscr{O}_{X}$-module that agrees with the first non-zero cohomology of a dualizing complex $\omega_{X}^{\cdot}$. In particular, for a normalized dualizing complex $\omega_{X}^{\cdot}$, its $(-\operatorname{dim} X)$-th cohomology $\omega_{X}:=\mathbf{h}^{-\operatorname{dim} X} \omega_{X}^{\cdot}$ is a canonical module; since it is the first non-zero cohomology there is a natural inclusion 
$\omega_{X}[\operatorname{dim} X] \hookrightarrow \omega_{X}^{\cdot}$. If $X$ is $\mathrm{S}_{1}$, i.e. satisfies Serre's first condition, then $\omega_{X}$ is $\mathrm{S}_{2}$ by Har07, Lemma 1.3]. Also see [KM98, Corollary 5.69] where it is shown that any (quasi-)projective scheme over a field has an $\mathrm{S}_{2}$ canonical module. We also note that if $X$ is integral, then $\omega_{X}$ can be taken to be the $\mathrm{S}_{2}$-module agreeing with the dualizing complex on the CohenMacaulay locus of $X$.

We shall make extensive use of the trace map from Grothendieck-Serre duality, see Con00, Har66]. For $S$ a base scheme, Noetherian, excellent, and separated, we consider the category $\operatorname{Sch}_{S}$ of $S$-schemes (essentially) of finite type over $S$, with $S$-morphisms between them. We assume as before that $S$ has a dualizing complex $\omega_{S}^{\cdot}$. Then Grothendieck duality theory provides us with a functor $f^{!}$for every $S$-morphism $f: Y \rightarrow X$ with the properties

(a) (_)! is compatible with composition, i.e. if $g: Z \rightarrow Y$ is a further $S$-morphism, then there is a natural isomorphism of functors $(f \circ g) ! \cong g^{!} \circ f^{!}$which is compatible with triple composition.

(b) If $f$ is of finite type, and $\omega_{X}^{\circ}$ is a dualizing complex on $X$ then $f^{!} \omega_{X}^{\cdot}$ is a dualizing complex on $Y$. If additionally $f$ is a dominant morphism of integral schemes and $\omega_{X}^{\bullet}$ is normalized, then $f^{!} \omega_{X}^{\cdot}$ is also normalized.

(c) If $f$ is a finite map, then $f^{!}\left(\boldsymbol{Z}_{\boldsymbol{Z}}\right)=\mathbf{R} \mathscr{H}_{\mathrm{O}} m_{\mathscr{O}_{X}}\left(f_{*} \mathscr{O}_{Y}, \ldots\right)$ viewed as a complex of $\mathscr{O}_{Y}$-modules. Note that the right hand side is defined for any finite morphism, not just for an $S$-morphism.

Therefore we may define for each $S$-scheme $X$ with structural map $\pi_{X}: X \rightarrow S$ the dualizing complex $\omega_{X}^{\circ}:=\pi_{X}^{!} \omega_{S}^{\cdot}$. After this choice of dualizing complexes on $\operatorname{Sch}_{S}$, the compatibility with composition now immediately implies that for any $S$-morphism $f: Y \rightarrow X$ we have a canonical isomorphism $f^{!} \omega_{X}^{\cdot} \cong \omega_{Y}^{\cdot}$.

Remark 2.9. In the remainder of the paper, the base scheme $S$ will typically either be a field, or it will be the scheme $X$ we are interested in. Note that in either case the absolute Frobenius map $F: X \rightarrow X$ is not a map of $S$-schemes with the obvious choice of (the same) structural maps. However, using the composition $F: X \rightarrow X \rightarrow S$, we do obtain a new $S$-scheme structure for $X$ and so we view $F: X \rightarrow X$ as a map of different $S$-schemes.

A key point in the construction of $({ })$ ! is that for $f: Y \rightarrow X$ proper there is a natural transformation of functors

$$
\mathbf{R} f_{*} f^{!} \rightarrow \operatorname{id}_{X}
$$

called the trace map which induces a natural isomorphism of functors in the derived category

$$
\mathbf{R} f_{*} \mathbf{R} \mathscr{H} o m_{Y}\left(\mathscr{M}^{\bullet}, f^{!} \mathscr{N}^{\bullet}\right) \rightarrow \mathbf{R} \mathscr{H}_{0 m_{Y}}\left(\mathbf{R} f_{*} \mathscr{M}^{\bullet}, \mathscr{N}^{\bullet}\right)
$$

for any bounded above complex of quasi-coherent $\mathscr{O}_{Y}$-modules $\mathscr{M}^{\bullet}$ and bounded below complex of coherent $\mathscr{O}_{X}$-modules $\mathscr{N}^{\bullet}$. This statement, which expresses that $f^{!}$is right adjoint to $\mathbf{R} f_{*}$ for proper $f$, is the duality theorem in its general form.

Applying trace map to the dualizing complex $\omega_{X}^{\cdot}$ we obtain

$$
\operatorname{Tr}_{f} \bullet: \mathbf{R} f_{*} \omega_{Y}^{\bullet} \cong \mathbf{R} f_{*} f^{!} \omega_{X}^{\cdot} \rightarrow \omega_{X}^{\cdot}
$$

which we also refer to as the trace map and denote by $\operatorname{Tr}_{f} \bullet$. Equivalently, by the duality theorem, the trace map is Grothendieck-Serre dual to the corresponding map of structure sheaves $f: \mathscr{O}_{X} \rightarrow \mathbf{R} f_{*} \mathscr{O}_{Y}$. The key properties of the trace relevant for us are

(a) Compatibility with composition, i.e. if $g: Z \rightarrow Y$ is another proper $S$-morphism then $\operatorname{Tr}_{(f \circ g)} \bullet=\operatorname{Tr}_{f} \bullet \circ \mathbf{R} f_{*} \operatorname{Tr}_{g} \bullet$.

(b) The trace map is compatible with certain base changes. In general this is a difficult and subtle issue (see [Con00]); however, we will only need this for open inclusions $U \subseteq X$ and localization at a point, where it is not problematic. 
(c) In the case that $f$ is finite, $\operatorname{Tr}_{f}$ is locally given by evaluation at 1 ,

$$
\operatorname{Tr}_{f} \bullet: \mathbf{R} f_{*} f^{!} \omega_{X}^{\cdot}=\mathbf{R} f_{*} \mathbf{R} \mathscr{H}_{0} m_{\mathscr{O}_{X}}\left(f_{*} \mathscr{O}_{Y}, \omega_{X}\right) \rightarrow \omega_{X}
$$

For a proper dominant morphism $\pi: Y \rightarrow X$ of integral schemes, the trace gives rise to maps on canonical modules as well (not just dualizing complexes). Taking the ( $-\operatorname{dim} X)$-th cohomology of $\operatorname{Tr}_{\pi} \cdot: \mathbf{R} \pi_{*} \omega_{Y}^{\cdot} \rightarrow \omega_{X}^{\cdot}$ gives

$$
\operatorname{Tr}_{\pi} \cdot: \mathbf{h}^{-\operatorname{dim} X}\left(\mathbf{R} \pi_{*} \omega_{Y}^{\cdot}\right) \rightarrow \mathbf{h}^{-\operatorname{dim} X} \omega_{X}^{\cdot}=: \omega_{X}
$$

which we will also denote by $\operatorname{Tr}_{\pi} \cdot$. Further composing with the inclusion $\omega_{Y}[\operatorname{dim} Y] \rightarrow \omega_{Y}^{\bullet}$ then gives

$$
\operatorname{Tr}_{\pi}: \mathbf{h}^{\operatorname{dim} Y-\operatorname{dim} X} \mathbf{R} \pi_{*} \omega_{Y} \rightarrow \mathbf{h}^{-\operatorname{dim} X} \mathbf{R} \pi_{*} \omega_{Y}^{\cdot} \stackrel{\operatorname{Tr}_{\pi} \cdot}{\longrightarrow} \omega_{X}
$$

which we also refer to as the trace map and now denote by $\operatorname{Tr}_{\pi}$. Note that the above construction remains compatible with localization on the base scheme, which we make use of below in showing under mild assumptions that this trace map is non-zero.

Proposition 2.13. If $\pi: Y \rightarrow X$ is a proper dominant morphism of integral schemes, then the trace map

$$
\operatorname{Tr}_{\pi}: \mathbf{h}^{\operatorname{dim} Y-\operatorname{dim} X} \mathbf{R} \pi_{*} \omega_{Y} \rightarrow \omega_{X}
$$

is non-zero.

Proof. To show the statement we may assume that $X=\operatorname{Spec} K$ for $K$ a field. The map $\mathbb{H}^{0}\left(Y, \omega_{Y}^{\cdot}\right)=\mathbf{h}^{0} \mathbf{R} \pi_{*} \omega_{Y}^{\cdot} \rightarrow \omega_{K}^{\cdot} \cong K$ is non-zero as it is Grothendieck-Serre dual to the natural inclusion $K \rightarrow H^{0}\left(Y, \mathscr{O}_{Y}\right)$. Hence it is sufficient to show that the map $\mathbf{h}^{\operatorname{dim} Y}\left(\mathbf{R} \pi_{*} \omega_{Y}\right) \rightarrow \mathbf{h}^{0}\left(\mathbf{R} \pi_{*} \omega_{Y}^{\bullet}\right)$ is surjective. If $Y$ is Cohen-Macaulay, i.e. $\omega_{Y}[\operatorname{dim} Y]=$ $\omega_{Y}^{\dot{ }}$, we are done. More generally consider the hypercohomology spectral sequence $E_{2}=$ $H^{i}\left(Y, \mathbf{h}^{j} \omega_{Y}^{\cdot}\right) \Rightarrow \mathbb{H}^{i+j}\left(Y, \omega_{Y}^{\bullet}\right)$. The only terms contributing to $\mathbb{H}^{0}\left(Y, \omega_{Y}^{\bullet}\right)$ are $H^{i}\left(Y, \mathbf{h}^{j} \omega_{Y}^{\bullet}\right)$ with $i+j=0$. In the next lemma, it is shown that $\operatorname{dim} \operatorname{supp} \mathbf{h}^{j} \omega_{Y}^{\cdot}<-j$ for $j>-\operatorname{dim} Y$, which hence implies that $H^{\operatorname{dim} Y}\left(Y, \omega_{Y}\right)=\mathbf{h}^{\operatorname{dim} Y}\left(\mathbf{R} \pi_{*} \omega_{Y}\right)$ is the only non-vanishing term among them and thus surjects onto $\mathbf{h}^{0}\left(\mathbf{R} \pi_{*} \omega_{Y}^{\cdot}\right)$ as claimed.

Lemma 2.14. Let $(R, \mathfrak{m})$ be an equidimensional local $\mathrm{S}_{1}$ ring of dimension $n$ with normalized dualizing complex $\omega_{R}^{\cdot}$. Then

$$
\operatorname{dim} \operatorname{supp} \mathbf{h}^{-j} \omega_{R}^{\cdot}<j
$$

for $j<n$.

Proof. By local duality $\mathbf{h}^{0} \omega_{R}^{\cdot}$ is Matlis dual to $\Gamma_{\mathfrak{m}}(R)$ which by the $\mathrm{S}_{1}$ condition is zero. This shows the lemma for $j=0$, and hence in particular for $n=1$, so that we may proceed by induction on $n$.

Assuming that $1 \leq j<n$, if $\operatorname{dim} \operatorname{supp} \mathbf{h}^{-j} \omega_{R}^{\cdot}=0$ we are done. Otherwise, we have $c=\operatorname{dimsupp} \mathbf{h}^{-j} \omega_{R}^{\cdot}>0$ and can take $\mathfrak{p} \neq \mathfrak{m}$ to be a minimal prime in the support of $\mathbf{h}^{-j} \omega_{R}^{\cdot}$ with $\operatorname{dim} R / \mathfrak{p}=c$. Since $\operatorname{dim} R_{\mathfrak{p}}=n-c<n$, we have that $\left(\omega_{R}^{\bullet}\right)_{\mathfrak{p}}[-c]$ is a normalized dualizing complex for $R_{\mathfrak{p}}$. Thus, by the induction hypothesis it follows

$$
0=\operatorname{dim} \operatorname{supp} \mathbf{h}^{-j}\left(\omega_{R}^{\bullet}\right)_{\mathfrak{p}}=\operatorname{dim} \operatorname{supp} \mathbf{h}^{-j+c}\left(\omega_{R}^{\bullet}\right)_{\mathfrak{p}}[-c]<j-c
$$

so that $c=\operatorname{dim} \operatorname{supp} \mathbf{h}^{-j} \omega_{R}^{\bullet}<j$ as desired.

We address now a particularly subtle issue surrounding the upper shriek functor and Frobenius. 
Example 2.15 (Trace of Frobenius and behavior of dualizing complexes). A particularly important setting in this paper is that of a scheme $X$ essentially of finite type over an $F$ finite base scheme $S$ of positive characteristic $p$ (e.g. a perfect field of characteristic $p>0$ ). This means simply that the (absolute) Frobenius or $p$-th power map $F: X \rightarrow X$ is a finite morphism, and hence proper. Thus we have the "evaluation at 1" trace map

$$
\operatorname{Tr}_{F} \cdot: F_{*} F^{!} \omega_{X}^{\cdot} \cong F_{*} \mathbf{R} \mathscr{H}_{0} m_{X}\left(F_{*} \mathscr{O}_{X}, \omega_{X}\right) \rightarrow \omega_{X}^{\bullet}
$$

However, note that since the Frobenius $F$ is generally not an $S$-morphism we do not have, a priori, that $F^{!} \omega_{X}^{\circ} \cong \omega_{X}^{\cdot}$. This needs an additional assumption, namely that this property holds for the base scheme $S$. Using a fixed isomorphism $F^{!} \omega_{\dot{S}}^{\bullet} \cong \omega_{S}^{\cdot}$, the compatibility of $(\ldots)$ with composition in the commutative diagram

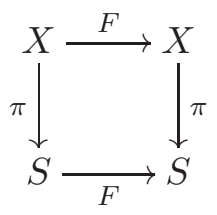

shows that indeed

$$
F^{!} \omega_{X} \cong F^{!} \pi^{!} \omega_{S}^{\cdot} \cong \pi^{!} F^{!} \omega_{S} \cong \pi^{!} \omega_{S}^{\cdot} \cong \omega_{X}^{\cdot}
$$

Convention 2.16. For simplicity, we will always assume that all our base schemes $S$ of positive characteristic $p$ are $F$-finite and satisfy $F^{!} \omega_{S}^{\bullet} \cong \omega_{S}^{\cdot}$ for the given choice of dualizing complex $\omega_{S}^{\cdot}$. This is automatic if $S$ is the spectrum of a local ring (e.g. a field).

Remark 2.17. The assumption $F^{!} \omega_{S}^{\bullet} \cong \omega_{S}^{\bullet}$ is convenient but could nonetheless be avoided. Notice that regardless, $F^{!} \omega_{S}^{\bullet}$ is a dualizing complex, and so it already agrees with $\omega_{S}^{\bullet}$ up to a shift and up to tensoring with an invertible sheaf. It is easy to see that the shift is zero since $F$ is a finite map, thus we have $F^{!} \omega_{S}^{\cdot} \cong \omega_{S}^{\cdot} \otimes_{\mathscr{O}_{S}} \mathscr{L}$ for some invertible sheaf $\mathscr{L}$. One option would be to carefully keep track of $\mathscr{L}$ throughout all constructions and arguments - this we chose to avoid. In any case, if one is willing to work locally over the base $S$ (as is the case with most of our main theorems) one may always assume that $F^{!} \omega_{\dot{S}}^{\cdot}=\omega_{S}^{\bullet}$ simply by restricting to charts where $\mathscr{L}$ is trivial.

Proposition 2.8 and Proposition 2.13 can be combined as follows.

Proposition 2.18. Suppose that $\pi: Y \rightarrow X$ is a proper dominant morphism between normal integral schemes, and let $\Delta$ be a $\mathbb{Q}$-divisor on $X$ such that $K_{X}+\Delta$ is $\mathbb{Q}$-Cartier. Then the trace map of $\pi$ induces a non-zero map

$$
\operatorname{Tr}_{\pi}: \mathbf{h}^{\operatorname{dim} Y-\operatorname{dim} X} \mathbf{R} \pi_{*} \omega_{Y}\left(-\left\lfloor\pi^{*}\left(K_{X}+\Delta\right)\right\rfloor\right) \rightarrow \omega_{X}\left(-\left\lfloor K_{X}+\Delta\right\rfloor\right) .
$$

Similarly, if additionally $\pi^{*}\left(K_{X}+\Delta\right)$ is a Cartier divisor, then we have another non-zero map

$$
\operatorname{Tr}_{\pi} \cdot: \mathbf{h}^{-\operatorname{dim} X} \mathbf{R} \pi_{*} \omega_{Y}\left(-\left\lfloor\pi^{*}\left(K_{X}+\Delta\right)\right\rfloor\right) \rightarrow \omega_{X}\left(-\left\lfloor K_{X}+\Delta\right\rfloor\right) .
$$

Proof. The difficulty here lies in that $\left\lfloor K_{X}+\Delta\right\rfloor$ need not be $\mathbb{Q}$-Cartier. To overcome this, let $U \stackrel{i}{\longleftrightarrow} X$ be the regular locus of $X$, then we have the trace map

$$
\mathbf{h}^{\operatorname{dim} Y-\operatorname{dim} X} \mathbf{R} \pi_{*} \omega_{\pi^{-1}(U)} \stackrel{\operatorname{Tr}_{\pi} \cdot}{\longrightarrow} \omega_{U}
$$

which is just the restriction of (2.12) to $U$. Tensoring this map by the invertible sheaf $\mathscr{O}_{U}\left(-\left\lfloor\left(K_{X}+\Delta\right)\right\rfloor\right)$, we have by the projection formula

$$
\mathbf{h}^{\operatorname{dim} Y-\operatorname{dim} X} \mathbf{R} \pi_{*} \omega_{\pi^{-1}(U)}\left(-\pi^{*}\left\lfloor\left(K_{X}+\Delta\right)\right\rfloor\right) \rightarrow \omega_{U}\left(-\left\lfloor\left(K_{X}+\Delta\right)\right\rfloor\right) .
$$


Since $-\pi^{*}\left\lfloor K_{X}+\Delta\right\rfloor \geq-\left\lfloor\pi^{*}\left(K_{X}+\Delta\right)\right\rfloor$ on $U$ (where again $\left\lfloor K_{X}+\Delta\right\rfloor$ is Cartier), we have an induced map

$$
\mathbf{h}^{\operatorname{dim} Y-\operatorname{dim} X} \mathbf{R} \pi_{*} \omega_{\pi^{-1}(U)}\left(-\left\lfloor\pi^{*}\left(K_{X}+\Delta\right)\right\rfloor\right) \rightarrow \omega_{U}\left(-\left\lfloor\left(K_{X}+\Delta\right)\right\rfloor\right) .
$$

Applying $i_{*}\left(\_\right)$to (2.19) and composing with the restriction map

$$
\mathbf{h}^{\operatorname{dim} Y-\operatorname{dim} X} \mathbf{R} \pi_{*} \omega_{Y}\left(-\left\lfloor\pi^{*}\left(K_{X}+\Delta\right)\right\rfloor\right) \rightarrow i_{*} \mathbf{h}^{\operatorname{dim} Y-\operatorname{dim} X} \mathbf{R} \pi_{*} \omega_{\pi^{-1}(U)}\left(-\left\lfloor\pi^{*}\left(K_{X}+\Delta\right)\right\rfloor\right)
$$

now gives the desired first map

$$
\operatorname{Tr}_{\pi}: \mathbf{h}^{-\operatorname{dim} X} \mathbf{R} \pi_{*} \omega_{Y}\left(-\left\lfloor\pi^{*}\left(K_{X}+\Delta\right)\right\rfloor\right) \rightarrow \omega_{X}\left(-\left\lfloor K_{X}+\Delta\right\rfloor\right)
$$

by noting that $i_{*} \omega_{U}\left(-\left\lfloor\left(K_{X}+\Delta\right)\right\rfloor\right)=\omega_{X}\left(-\left\lfloor\left(K_{X}+\Delta\right)\right\rfloor\right)$. To see that $\operatorname{Tr}_{\pi}$ is non-zero, localize to the generic point of $X$ (where $K_{X}+\Delta$ is trivial) and apply Proposition 2.13.

The construction of the second map $\operatorname{Tr}_{\pi}$ • is similar, rather starting from (2.11) in place of (2.12). Notice that we require that $\pi^{*}\left(K_{X}+\Delta\right)$ to be Cartier so that we have a means of interpreting $\omega_{Y}^{\bullet}\left(-\left\lfloor\pi^{*}\left(K_{X}+\Delta\right)\right\rfloor\right)$. Since $\operatorname{Tr}_{\pi} \cdot$ agrees with $\operatorname{Tr}_{\pi}$ generically by the proof of Proposition 2.13, this map is again non-zero.

In the previous Proposition, we studied trace maps twisted by $\mathbb{Q}$-divisors. In the next Lemma, we study a special case of this situation which demonstrates that sometimes this trace map can be re-interpreted as generating a certain module of homomorphisms.

Lemma 2.20. Let $X$ be a normal integral $F$-finite scheme. Suppose that $\Delta$ is a $\mathbb{Q}$-divisor such that $\left(p^{e}-1\right)\left(K_{X}+\Delta\right)=\operatorname{div} c$ for some $e>0$ and $0 \neq c \in K(X)$. If $\Phi_{X}^{e}: F^{e} \omega_{X} \rightarrow \omega_{X}$ is the trace of the e-iterated Frobenius, then the homomorphism $\phi\left({ }_{-}\right)=\Phi_{X}^{e}\left(F_{*}^{e} c \cdot{ }_{-}\right)$ generates $\operatorname{Hom}_{\mathscr{O}_{X}}\left(F_{*}^{e} \mathscr{O}_{X}\left(\left\lceil\left(p^{e}-1\right) \Delta\right\rceil\right), \mathscr{O}_{X}\right)$ as an $F_{*}^{e} \mathscr{O}_{X}$-module.

Proof. Essentially by construction (and the definition of $\omega_{X}^{\cdot}$ ), we have that $\Phi_{X}^{e}$ generates $\operatorname{Hom}_{\mathscr{O}_{X}}\left(F_{*}^{e} \omega_{X}, \omega_{X}\right)$ as an $F_{*}^{e} \mathscr{O}_{X}$-module. Using the identification $\omega_{X}=\mathscr{O}_{X}\left(K_{X}\right)$ we may consider $\Phi_{X}^{e}$ to generate

$$
\operatorname{Hom}_{\mathscr{O}_{X}}\left(F_{*}^{e} \mathscr{O}_{X}\left(\left(1-p^{e}\right) K_{X}\right), \mathscr{O}_{X}\right)=\operatorname{Hom}_{\mathscr{O}_{X}}\left(F_{*}^{e} \omega_{X}, \omega_{X}\right)
$$

But then multiplication by $c$ induces an isomorphism $\mathscr{O}_{X}\left(\left(p^{e}-1\right) \Delta\right) \stackrel{\cdot c}{\longrightarrow} \mathscr{O}_{X}\left(\left(1-p^{e}\right) K_{X}\right)$ (note $\left(p^{e}-1\right) \Delta$ is integral), so that $\Phi_{X}\left(F_{*}^{e} c \cdot \_\right)$generates $\operatorname{Hom}_{\mathscr{O}_{X}}\left(F_{*}^{e} \mathscr{O}_{X}\left(\left\lceil\left(p^{e}-1\right) \Delta\right\rceil\right), \mathscr{O}_{X}\right)$ as an $F_{*}^{e} \mathscr{O}_{X}$-module as well.

A main technique in this paper is the observation that the images of the various trace maps $\operatorname{Tr}_{\pi}$ are preserved under the trace of the Frobenius. We will show this now for $\operatorname{Tr}_{\pi}: \mathbf{h}^{\operatorname{dim} Y-\operatorname{dim} X} R \pi_{*} \omega_{Y} \rightarrow \omega_{X}$. We will obtain a partial generalization involving $\mathbb{Q}$ divisors within the proof of Proposition 4.2.

Proposition 2.21. If $\pi: Y \rightarrow X$ is a proper dominant map of integral schemes, the image of the trace map

$$
J_{\pi}:=\operatorname{Tr}_{\pi}\left(\mathbf{h}^{\operatorname{dim} Y-\operatorname{dim} X} \mathbf{R} \pi_{*} \omega_{Y}\right) \subseteq \omega_{X}
$$

satisfies $\Phi_{X}\left(F_{*} J_{\pi}\right) \subseteq J_{\pi}$.

Proof. Since Frobenius commutes with any map, we get the following diagram for which we consider the corresponding commutative diagram of structure sheaves
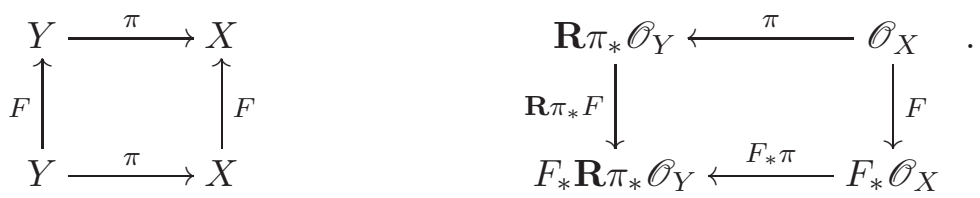
Applying duality now gives the following commutative diagram of trace maps

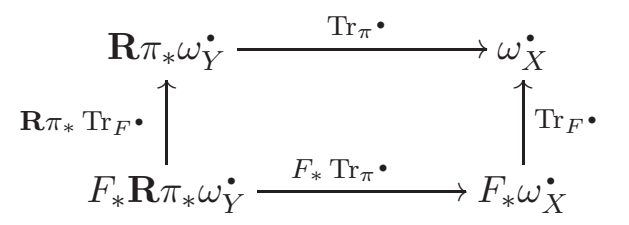

Taking the $(-\operatorname{dim} X)$-th cohomology and composing with the inclusion $\omega_{Y}[\operatorname{dim} Y] \rightarrow \omega_{Y}^{\cdot}$ on the left, we get a diagram

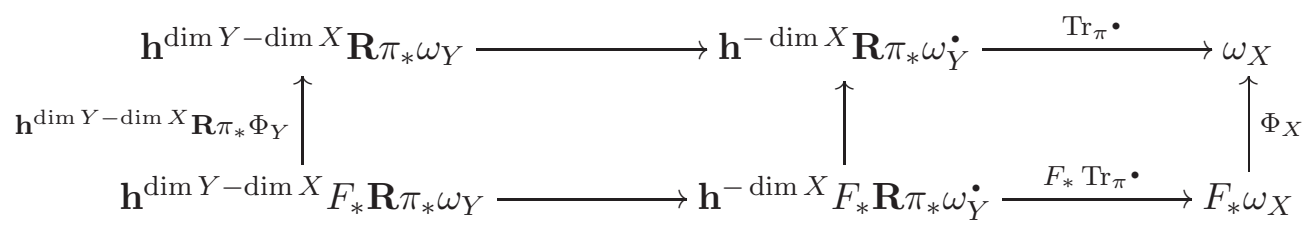

where the left vertical map exists since $F$ is finite and hence $F_{*}$ is exact. The horizontal composition on the top is $\operatorname{Tr}_{\pi}$ and the image $\operatorname{Tr}_{\pi}\left(\mathbf{h}^{\operatorname{dim} Y-\operatorname{dim} X} R \pi_{*} \omega_{Y}\right)$ is $J_{\pi} \subseteq \omega_{X}$. By the exactness of $F_{*}$, the horizontal composition on the bottom is $F_{*} \operatorname{Tr}_{\pi}$ and we get that $F_{*} J_{\pi}$ is the image, and the result now follows.

\subsection{Multiplier ideals and pseudo-rationality.}

Definition 2.24. [LT81] We say that a reduced connected scheme $X$ is pseudo-rational if

(a) $X$ is Cohen-Macaulay, and

(b) $\pi_{*} \omega_{Y}=\omega_{X}$ for every proper birational map $\pi: Y \rightarrow X$.

Furthermore, if there exists a resolution of singularities $\pi: Y \rightarrow X$, then it is sufficient to check (b) for this one map $\pi$.

If $X$ is of characteristic zero, this coincides via Grauert-Riemenschneider vanishing [GR70] with the classical definition of rational singularities, meaning there exists a resolution of singularities $\pi: Y \rightarrow X$ such that $\mathscr{O}_{X} \cong \pi_{*} \mathscr{O}_{Y}$ and $\mathbf{h}^{i} \mathbf{R} \pi_{*} \mathscr{O}_{Y}=0$ for all $i$. In positive or mixed characteristic, it is a distinct notion.

Remark 2.25. It was remarked in [GR70] that if $\pi: Y \rightarrow X$ is a resolution of singularities in characteristic zero, then the subsheaf $\pi_{*} \omega_{Y} \subseteq \omega_{X}$ is independent of the choice of resolution of singularities. This subsheaf should be viewed as an early version of the multiplier ideal. Compare with the definition of the multiplier module below and the parameter test submodule in Definition 2.33,

Going back to ideas in K. Smith's thesis and Smi95, the natural object to deal with rational singularities of pairs is the multiplier module, $c f$. Bli04, ST08.

Definition 2.26. Given a pair $(X, \Gamma)$ with $\Gamma$ a $\mathbb{Q}$-Cartier $\mathbb{Q}$-divisor, then the multiplier module is defined as

$$
\mathcal{J}\left(\omega_{X} ; \Gamma\right):=\bigcap_{\pi: Y \rightarrow X} \pi_{*} \omega_{Y}\left(\left\lceil-\pi^{*} \Gamma\right\rceil\right)
$$

where $\pi$ ranges over all proper birational maps with normal $Y$.

Note that from this definition it is not clear that $\mathcal{J}\left(\omega_{X} ; \Gamma\right)$ is even quasi-coherent, as the infinite intersection of coherent subsheaves need not be quasi-coherent in general. However, if there is a theory of resolution of singularities available (for example over a field of characteristic zero [Hir64], or in dimension $\leq 2$ [Lip78]), it is straightforward to check coherence by showing that the above intersection stabilizes. Recall that a log resolution of 
the pair $(X, \Gamma)$ is a proper birational map $\pi: Y \rightarrow X$ with $Y$ regular and exceptional set $E$ of pure codimension one such that $\operatorname{Supp}(E) \cup \pi^{-1}(\operatorname{Supp}(\Gamma))$ is a simple normal crossings divisor. Assuming every normal proper birational modification can be dominated by a log resolution, one can in fact show

$$
\mathcal{J}\left(\omega_{X} ; \Gamma\right)=\pi_{*} \omega_{Y}\left(\left\lceil-\pi^{*} \Gamma\right\rceil\right)
$$

for any single $\log$ resolution $\pi: Y \rightarrow X$, which is in particular coherent. Note that, for effective $\Gamma$ it is a subsheaf of $\omega_{X}$ via the natural inclusion $\pi_{*} \omega_{Y} \subseteq \omega_{X}$ as in Example 2.1.

Immediately from this definition it follows that $X$ is pseudo-rational if and only if $X$ is Cohen-Macaulay and $\mathcal{J}\left(\omega_{X}\right):=\mathcal{J}\left(\omega_{X} ; 0\right)=\omega_{X}$. Hence one defines:

Definition 2.27 ([ST08]). A pair $(X, \Gamma)$ with $\Gamma \geq 0$ a $\mathbb{Q}$-Cartier $\mathbb{Q}$-divisor is called pseudorational if $X$ is Cohen-Macaulay and $\mathcal{J}\left(\omega_{X} ; \Gamma\right)=\omega_{X}$. Note that this implies that $\lfloor\Gamma\rfloor=0$.

The classical notion is of course that of multiplier ideals, which have been defined primarily in characteristic zero. See Laz04 for a complete treatment in this setting. Historically, while multiplier ideals first appeared in more analytic contexts and were originally defined using integrability conditions, one facet of their pre-history was defined for any normal integral scheme - J. Lipman's adjoint ideals Lip94. The definition we give here (which makes sense in arbitrary characteristic) is a slight generalization of Lipman's definition to the modern setting of pairs.

Definition 2.28 ([Laz04, Lip94]). Given a log- $\mathbb{Q}$-Gorenstein pair $(X, \Delta)$ then the multiplier ideal is defined as

$$
\mathcal{J}(X ; \Delta):=\bigcap_{\pi: Y \rightarrow X} \pi_{*} \mathscr{O}_{Y}\left(\left\lceil K_{Y}-\pi^{*}\left(K_{X}+\Delta\right)\right\rceil\right)
$$

where $\pi$ ranges over all proper birational maps with normal $Y$ and, for each individual $\pi$, we have that $K_{X}$ and $K_{Y}$ agree wherever $\pi$ is an isomorphism.

As above, in a non-local setting, for $\mathcal{J}(X ; \Delta)$ to be quasi-coherent one needs a good theory of resolution of singularities. In this situation,

$$
\mathcal{J}(X ; \Delta)=\pi_{*} \mathscr{O}_{Y}\left(\left\lceil K_{Y}-\pi^{*}\left(K_{X}+\Delta\right)\right\rceil\right)
$$

for any $\log$ resolution $\pi: Y \rightarrow X$ of the pair $(X, \Delta)$. In general, $\mathcal{J}(X ; \Delta)$ depends heavily on $\Delta$ and not simply the corresponding linear or $\mathbb{Q}$-linear equivalence class; a similar observation holds for the multiplier module as well.

If $(X, \Delta)$ is a pair, strictly speaking the object $\mathcal{J}\left(\omega_{X} ; K_{X}+\Delta\right)$ is ambiguous as $K_{X}$ is not uniquely determined (and represents a linear equivalence class of divisors). Nonetheless, for each choice of $K_{X}$ we have that $\mathcal{J}\left(\omega_{X} ; K_{X}+\Delta\right) \subseteq \omega_{X}\left(-\left\lfloor K_{X}+\Delta\right\rfloor\right)$, and is thereby identified with a submodule of $\mathscr{O}_{X}(-\lfloor\Delta\rfloor)$ using Convention 2.7. This construction is in fact independent of the choice of $K_{X}$, and allows one to relate multiplier ideals and multiplier modules in general.

Lemma 2.29. If $(X, \Delta)$ is a log- $\mathbb{Q}$-Gorenstein pair, then $\mathcal{J}\left(\omega_{X} ;\left(K_{X}+\Delta\right)\right)=\mathcal{J}(X ; \Delta)$.

Proof. Suppose $\pi: Y \rightarrow X$ is a proper birational map, $Y$ is normal, and $K_{Y}$ and $K_{X}$ agree wherever $\pi$ is an isomorphism. Making full use of Convention 2.7, we have

$$
\begin{array}{ccc}
\omega_{X}\left(-\left\lfloor K_{X}+\Delta\right\rfloor\right) & = & \mathscr{O}_{X}(-\lfloor\Delta\rfloor) \\
\cup । & \cup l \\
\pi_{*} \omega_{Y}\left(-\left\lfloor\pi^{*}\left(K_{X}+\Delta\right)\right\rfloor\right) & = & \pi_{*} \mathscr{O}_{Y}\left(\left\lceil K_{Y}-\pi^{*}\left(K_{X}+\Delta\right)\right\rceil\right)
\end{array}
$$

and the desired conclusion now follows immediately from the definitions. 
Definition 2.30. A log- $\mathbb{Q}$-Gorenstein pair $(X, \Delta)$ with $\Delta \geq 0$ effective is called Kawamata log terminal if $\mathcal{J}(X ; \Delta)=\mathscr{O}_{X}$.

2.5. The parameter test submodule and $F$-rationality. We now turn to the characteristic $p>0$ notion of $F$-rationality, extensively studied in [FW89] and Smi97b], which is central to our investigations.

Definition 2.31. Suppose that $X$ is reduced, connected, and $F$-finite (and satisfies Convention 2.17). We say that $X$ is F-rational if

(a) $X$ is Cohen-Macaulay.

(b) There is no proper submodule $M \subseteq \omega_{X}$, non-zero on every irreducible component of $X$ where $\omega_{X}$ is non-zero, such that $\Phi_{X}\left(F_{*} M\right) \subseteq M$ where $\Phi_{X}: F_{*} \omega_{X} \rightarrow \omega_{X}$ is the trace of Frobenius as in Example 2.4.

Remark 2.32. While the preceding characterization of $F$-rationality differs from the definition used historically throughout the literature, it is nonetheless readily seen to be equivalent. Indeed, when $X=\operatorname{Spec} R$ for a local ring $R$ with maximal ideal $\mathfrak{m}$, the characterization of the tight closure of zero in $H_{\mathfrak{m}}^{\operatorname{dim} R}(R)$ found in Smi97b] implies that condition (b) is Matlis dual to the statement $0_{H_{\mathfrak{m}}^{\operatorname{dim} R}(R)}^{*}=0$.

Definition 2.33 ([Smi95, Bli04, ST08]). Suppose that $X$ is reduced, connected, and $F$-finite (and satisfies Convention 2.17). The parameter test submodule $\tau\left(\omega_{X}\right)$ is the unique smallest subsheaf $M$ of $\omega_{X}$, non-zero on every irreducible component of $X$ where $\omega_{X}$ is nonzero, such that $\Phi_{X}\left(F_{*} M\right) \subseteq M$ where $\Phi_{X}: F_{*} \omega_{X} \rightarrow \omega_{X}$ is trace of Frobenius.

For $X$ normal and integral, the parameter test submodule $\tau\left(\omega_{X} ; \Gamma\right)$ of a pair $(X, \Gamma)$ with $\Gamma \geq 0$ is the unique smallest non-zero subsheaf $M$ of $\omega_{X}$ such that $\phi\left(F_{*}^{e} M\right) \subseteq M$ for all local sections $\phi \in \mathscr{H}_{0} m_{\mathscr{O}_{X}}\left(F_{*}^{e} \omega_{X}\left(\left\lceil\left(p^{e}-1\right) \Gamma\right\rceil\right), \omega_{X}\right)$ and all $e>0$, noting that $\omega_{X} \subseteq \omega_{X}\left(\left\lceil\left(p^{e}-1\right) \Gamma\right\rceil\right)$. The further observation $\phi\left(F_{*} \omega_{X}(\lceil-\Gamma\rceil)\right) \subseteq \omega_{X}(\lceil-\Gamma\rceil)$ gives that $\tau\left(\omega_{X} ; \Gamma\right) \subseteq \omega_{X}(\lceil-\Gamma\rceil)$.

Once again, the preceding definition is separate from but equivalent to that which is commonly used throughout the literature. Moreover, standard arguments on the existence of test elements are required to show the (non-obvious) fact that $\tau\left(\omega_{X}\right)$ and $\tau\left(\omega_{X} ; \Gamma\right.$ ) (as above) are well-defined. See, for example, Sch11, Proposition 3.21] (cf. [Sch10, Lemma 2.17]), or more generally [BB11, Bli13].

Lemma 2.34. Suppose that $X$ is reduced, connected, and $F$-finite. Then $X$ is F-rational if and only if it is Cohen-Macaulay and $\tau\left(\omega_{X}\right)=\omega_{X}$. Furthermore, any F-rational scheme is normal.

Proof. The first statement is an immediate consequence of the definitions, so we need only show the second. Without loss of generality, we may assume that $X=\operatorname{Spec} R$ where $R$ is an $F$-rational local ring. If $R^{\mathrm{N}}$ is the normalization of $R$, we will show the inclusion map $i: R \rightarrow R^{\mathrm{N}}$ is an isomorphism. As $R$ is already assumed Cohen-Macaulay it is $\mathrm{S}_{2}$, and so by Serre's criterion for normality we simply need to check that $R$ is regular in codimension 1. Thus, by localizing we may assume that $R$ is one dimensional (and thus so is $R^{\mathrm{N}}$, which now must be regular). Consider the following commutative diagram of rings together with its corresponding Grothendieck-Serre dual (all rings in question are Cohen-Macaulay)
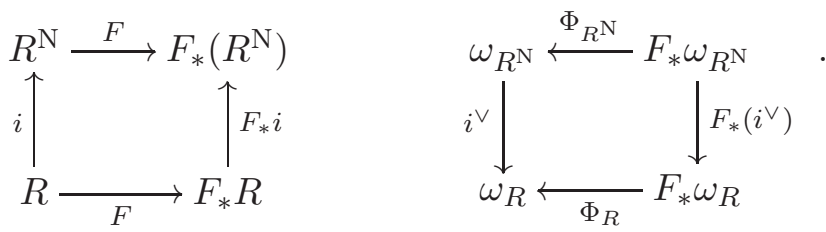
As in Example 2.2, $i^{\vee}$ is identified with the evaluation-at-1 map $\operatorname{Hom}_{R}\left(R^{\mathrm{N}}, \omega_{R}\right) \rightarrow \omega_{R}$. Since $i$ is birational, it is easy to see $i^{\vee}$ is injective. In particular, $i^{\vee}$ is non-zero and thus also surjective by the definition of $F$-rationality. It follows that $i^{\vee}$ and hence $i$ are isomorphisms, whence $R$ is normal as desired.

In order to consider pairs $(X, \Delta)$ with $\Delta$ not necessarily effective, we need to recall the following lemma.

Lemma 2.35. [ST08, Proposition 7.10(3)] ST10, Lemma 6.12] Suppose that $(X, \Delta)$ is a pair with $\Delta \geq 0$, and that $D \geq 0$ an integral Cartier divisor on $X=\operatorname{Spec} R$, then $\tau\left(\omega_{X} ; \Delta+D\right)=$ $\tau\left(\omega_{X} ; \Delta\right) \otimes \mathscr{O}_{X}(-D)$.

Definition 2.36. Suppose that $(X, \Gamma)$ is a pair. Fix a Cartier divisor $D$ on $X$ such that $\Gamma+D$ is effective (these always exist on affine charts). Then the parameter test module of $(X, \Gamma)$ is defined as

$$
\tau\left(\omega_{X} ; \Gamma\right):=\tau\left(\omega_{X} ; \Gamma+D\right) \otimes \mathscr{O}_{X}(D) .
$$

It is also easy to verify that this definition is independent of the choice of $D$, hence our local definition globalizes. It is straightforward to check that $\tau\left(\omega_{X} ; \Gamma\right) \subseteq \omega_{X}(\lceil-\Gamma\rceil)$.

In defining the test ideal of a pair below, we handle the non-effective case analogously.

Definition 2.37. Suppose that $X$ is reduced and $F$-finite. The test ideal $\tau(X)$ is the unique smallest ideal $J$ of $\mathscr{O}_{X}$, non-zero on every irreducible component of $X$, such that $\phi\left(F_{*}^{e} J\right) \subseteq J$ for every local section $\phi \in \mathscr{H}_{0} m_{\mathscr{O}_{X}}\left(F_{*}^{e} \mathscr{O}_{X}, \mathscr{O}_{X}\right)$ and all $e>0$.

If $(X, \Delta)$ is a pair with $\Delta \geq 0$, the test ideal $\tau(X ; \Delta)$ is the unique smallest non-zero ideal $J$ of $\mathscr{O}_{X}$ such that $\phi\left(F_{*}^{e} J\right) \subseteq J$ for any local section $\phi \in \mathscr{H}_{0} m_{\mathscr{O}_{X}}\left(F_{*}^{e} \mathscr{O}_{X}\left(\left\lceil\left(p^{e}-1\right) \Delta\right\rceil\right), \mathscr{O}_{X}\right)$ and all $e>0$, noting that $J \subseteq \mathscr{O}_{X} \subseteq \mathscr{O}_{X}\left(\left\lceil\left(p^{e}-1\right) \Delta\right\rceil\right)$.

As with the parameter test module, one has for any effective integral Cartier Divisor $D$ the equality $\tau(X ; \Delta+D)=\tau(X ; \Delta) \otimes \mathscr{O}_{X}(-D)$, which allows one to extend the definition to the non-effective case as above (see [ST08] for further details). Furthermore, the same subtle albeit well-known arguments are again required to show these ideals exist Sch11, Proposition 3.21] (see also [ST12a]).

Remark 2.38. As before, the preceding definition is non-standard; rather, what we have just defined is an alternative yet equivalent characterization of the big or non-finitistic test ideal, commonly denoted in the literature by $\tau_{b}(X, \Delta)$ or $\tilde{\tau}(X, \Delta)$. However, in many situations (and conjecturally in general) the big test ideal agrees with the classically defined or finitistic test ideal. Indeed, these two notions are known to coincide whenever $K_{X}+\Delta$ is $\mathbb{Q}$-Cartier Tak04, BSTZ10] - the only setting considered in this paper. For this reason, as well as our belief that the big test ideal is the correct object of study in general, we will drop the adjective big from the terminology throughout.

Strictly speaking the object $\tau\left(\omega_{X} ; K_{X}+\Delta\right)$ is ambiguous as $K_{X}$ is not uniquely determined. Indeed supposing $\Delta \geq 0$, as seen from Lemma 2.35, different choices of $K_{X}$ give rise to different submodules $\tau\left(\omega_{X} ; K_{X}+\Delta\right)$ of $\omega_{X}$. However, we in fact have $\tau\left(\omega_{X} ; K_{X}+\Delta\right) \subseteq$ $\omega_{X}\left(-\left\lfloor K_{X}+\Delta\right\rfloor\right)$, so that $\tau\left(\omega_{X} ; K_{X}+\Delta\right)$ is identified with a submodule of $\mathscr{O}_{X}(-\lfloor\Delta\rfloor)$ using Convention 2.7. As was the case with the multiplier module, this construction is independent of the choice of $K_{X}$.

Lemma 2.39. If $X$ is an $F$-finite and $(X, \Delta)$ is a pair, then $\tau\left(\omega_{X} ; K_{X}+\Delta\right)=\tau(X ; \Delta)$.

Proof. Choose a rational section $s \in \omega_{X}$ determining a canonical divisor $K_{X}=K_{X, s}=\operatorname{div} s$ and the embedding $\omega_{X} \subseteq \omega_{X} \otimes K(X) \stackrel{s \mapsto 1}{\longrightarrow} K(X)$. We will write $\omega_{X}=\mathscr{O}_{X}\left(K_{X}\right)$ for the 
duration of the proof without further remark. Working locally, it is harmless to assume $K_{X}$ and $\Delta$ are both effective by Definition 2.36, so that $\tau\left(\omega_{X} ; K_{X}+\Delta\right) \subseteq \omega_{X}\left(-\left\lfloor K_{X}+\Delta\right\rfloor\right)=$ $\mathscr{O}_{X}(-\lfloor\Delta\rfloor)$ gives in particular $\tau\left(\omega_{X} ; K_{X}+\Delta\right) \subseteq \mathscr{O}_{X} \subseteq \omega_{X}$

Next, observe for all $e>0$ that

$$
\mathscr{H}_{0 m_{\mathscr{O}_{X}}}\left(F_{*}^{e} \omega_{X}\left(\left\lceil\left(p^{e}-1\right)\left(K_{X}+\Delta\right)\right\rceil\right), \omega_{X}\right)=\mathscr{H}_{0} m_{\mathscr{O}_{X}}\left(F_{*}^{e} \mathscr{O}_{X}\left(\left\lceil\left(p^{e}-1\right) \Delta\right\rceil\right), \mathscr{O}_{X}\right)
$$

in a very precise sense; they are equal after mapping to the corresponding stalks at the generic point of $X$, which are explicitly identified with one another. Said another way, the image of $F_{*}^{e} \mathscr{O}_{X}\left(\left\lceil\left(p^{e}-1\right) \Delta\right\rceil\right) \subseteq F_{*}^{e} \omega_{X}\left(\left\lceil\left(p^{e}-1\right)\left(K_{X}+\Delta\right)\right\rceil\right)$ under every local homomorphism $\phi \in \mathscr{H}_{0} m_{\mathscr{O}_{X}}\left(F_{*}^{e} \omega_{X}\left(\left\lceil\left(p^{e}-1\right)\left(K_{X}+\Delta\right)\right\rceil\right), \omega_{X}\right)$ satisfies $\phi\left(F_{*}^{e} \mathscr{O}_{X}\left(\left\lceil\left(p^{e}-1\right) \Delta\right\rceil\right)\right) \subseteq \mathscr{O}_{X}$, giving rise to a commutative diagram

$$
\begin{aligned}
& F_{*}^{e} \omega_{X} \subseteq F_{*}^{e} \omega_{X}\left(\left\lceil\left(p^{e}-1\right)\left(K_{X}+\Delta\right)\right\rceil\right) \stackrel{\phi}{\longrightarrow} \omega_{X} \\
& \text { UI UI UI } \\
& F_{*}^{e} \mathscr{O}_{X} \subseteq \quad F_{*}^{e} \mathscr{O}_{X}\left(\left\lceil\left(p^{e}-1\right) \Delta\right\rceil\right) \stackrel{\phi}{\longrightarrow} \mathscr{O}_{X}
\end{aligned}
$$

and uniquely accounting for every local homomorphism in $\mathscr{H}_{0} m_{\mathscr{O}_{X}}\left(F_{*}^{e} \mathscr{O}_{X}\left(\left\lceil\left(p^{e}-1\right) \Delta\right\rceil\right), \mathscr{O}_{X}\right)$.

The desired conclusion is now an exercise in manipulating definitions. As $\tau(X ; \Delta) \subseteq \omega_{X}$ is preserved under the local homomorphisms in $\mathscr{H}_{0} m_{\mathscr{O}_{X}}\left(F_{*}^{e} \omega_{X}\left(\left\lceil\left(p^{e}-1\right)\left(K_{X}+\Delta\right)\right\rceil\right), \omega_{X}\right)$, we must have $\tau\left(\omega_{X} ; K_{X}+\Delta\right) \subseteq \tau(X ; \Delta)$ by the definition of $\tau\left(\omega_{X} ; K_{X}+\Delta\right)$. Conversely, as $\tau\left(\omega_{X} ; K_{X}+\Delta\right) \subseteq \mathscr{O}_{X}$ is preserved under $\mathscr{H}_{o m_{\mathscr{O}_{X}}}\left(F_{*}^{e} \mathscr{O}_{X}\left(\left\lceil\left(p^{e}-1\right) \Delta\right\rceil\right), \mathscr{O}_{X}\right)$, we must have $\tau(X ; \Delta) \subseteq \tau\left(\omega_{X} ; K_{X}+\Delta\right)$ and the statement follows.

2.6. Transformation behavior of test ideals and multiplier ideals. One of the contributions of this paper is a further clarification of the transformation behavior of test and multiplier ideals. Let us summarize what is known so far for alterations, which can always be viewed as compositions of proper birational maps and finite dominant maps.

Let us first consider the classical (and transparent) case of the multiplier ideal in characteristic zero [Laz04, 9.5.42]. Essentially by definition, the multiplier ideal of a log$\mathbb{Q}$-Gorenstein pair $\left(X, \Delta_{X}\right)$ is well-behaved under a proper birational morphism $\pi: Y \rightarrow X$ and satisfies

$$
\pi_{*} \mathcal{J}\left(Y, \Delta_{Y}\right)=\mathcal{J}\left(X, \Delta_{X}\right) \quad \text { with } \quad \Delta_{Y}:=\pi^{*}\left(K_{X}+\Delta_{X}\right)-K_{Y}
$$

where we have arranged that $\pi_{*} K_{Y}=K_{X}$ as usual. If rather $\pi: Y \rightarrow X$ is a finite dominant map, one sets $K_{Y}=\pi^{*} K_{X}+\operatorname{Ram}_{\pi}$ where $\operatorname{Ram}_{\pi}$ is the ramification divisor, and has the transformation rule

$\pi_{*} \mathcal{J}\left(Y, \Delta_{Y}\right) \cap K(X)=\mathcal{J}\left(X, \Delta_{X}\right) \quad$ with $\quad \Delta_{Y}:=\pi^{*}\left(K_{X}+\Delta_{X}\right)-K_{Y}=\pi^{*} \Delta_{X}-\mathrm{Ram}_{\pi}$.

In Section 8, we further generalize this rule to incorporate the trace map Example 2.2

$$
\operatorname{Tr}_{\pi}\left(\pi_{*} \mathcal{J}\left(Y, \Delta_{Y}\right)\right)=\mathcal{J}\left(X, \Delta_{X}\right)
$$

in the process of showing our main theorem in characteristic zero.

In characteristic $p>0$, the transformation rule (2.40) for the multiplier ideal under proper birational maps once again follows immediately from the definition. However, the behavior of the multiplier ideal for finite maps is more complicated and not fully understood in general - even for finite separable maps. In Section 8, we will show that both (2.41) and (2.42) hold for separable finite maps of degree prime to $p$ (and more generally when $\operatorname{Tr}_{\pi}\left(\pi_{*} \mathscr{O}_{Y}\right)=\mathscr{O}_{X}$ ). However, Examples 3.10, 6.13, and 7.12 in [ST10] together show that neither formula is valid for arbitrary separable finite maps in general. 
In contrast, the last two authors in [ST10] have completely described the behavior of the test ideal of a pair $\left(X, \Delta_{X}\right)$ under arbitrary finite dominant maps $\pi: Y \rightarrow X$. In the separable case, one again has

$$
\operatorname{Tr}_{\pi}\left(\pi_{*} \tau\left(Y, \Delta_{Y}\right)\right)=\tau\left(X, \Delta_{X}\right) \quad \text { with } \quad \Delta_{Y}:=\pi^{*}\left(K_{X}+\Delta_{X}\right)-K_{Y}=\pi^{*} \Delta_{X}-\operatorname{Ram}_{\pi} .
$$

More generally, when $\pi$ is not necessarily separable, a similar description holds after reinterpretation of the ramification divisor (via the Grothendieck trace). However, a formula as simple as (2.40) relating test ideals under a birational map cannot hold. Indeed, if $\pi: Y \rightarrow X$ is a $\log$ resolution of $\left(X, \Delta_{X}\right)$, then the multiplier and test ideal of $\left(Y, \Delta_{Y}\right)$ will agree while those of $\left(X, \Delta_{X}\right)$ may not ( $c f$. Tak04, Theorems 2.13, 3.2]). Nonetheless in Theorem 6.8 we will give a transformation rule - albeit far more complex - for the test ideal under alterations in general, so in particular for birational morphisms.

In summary we observe that the test ideal behaves well under finite maps (and may be computed using either finite maps or alterations by Theorem 4.6), whereas its behavior under birational maps is much more subtle. On the other hand, the multiplier ideal in characteristic zero behaves well under finite and birational maps, however finite maps will not suffice for its computation. The multiplier ideal in positive characteristic is still well behaved under birational transformations, but its behavior under finite maps is more subtle.

\section{F-RAtionality via alterations}

In this section, we will characterize $F$-rationality and, more generally, the parameter test submodule in terms of alterations. This is - at the same time - a special case of our Main Theorem as well as a key ingredient in its proof. The full proof of our Main Theorem in positive characteristic consists of a reduction to the cases treated here and will follow in the next section.

The crux of the argument to follow is based on a version of the equational lemma of [HH92] in the form that is found in HL07. In fact, we require a variant with an even stronger conclusion; namely, that the guaranteed finite extension may be assumed separable. This generalization follows from a recent result of A. Sannai and A. Singh [SS12].

Lemma 3.1 (equational lemma). Consider a domain $R$ with characteristic $p>0$. Let $K$ be the fraction field of $R, \bar{K}$ an algebraic closure of $K$, and $I$ an ideal of $R$. Suppose $i \geq 0$ and $\alpha \in H_{I}^{i}(R)$ is such that $\alpha, \alpha^{p}, \alpha^{p^{2}}, \ldots$ belong to a finitely generated $R$-submodule of $H_{I}^{i}(R)$. Then there exists a separable $R$-subalgebra $R^{\prime}$ of $\bar{K}$ that is a finite $R$-module such that the induced map $H_{I}^{i}(R) \rightarrow H_{I}^{i}\left(R^{\prime}\right)$ sends $\alpha$ to zero.

Proof. The statement of the equational lemma in [HL07, Lemma 2.2] is the same as above without the desired separability. Applying this weaker version we therefore have a finite extension $R \subseteq R^{\prime}$ such that $H_{I}^{i}(R) \rightarrow H_{I}^{i}\left(R^{\prime}\right)$ maps $\alpha$ to zero. Let $R^{s}$ be the separable closure of $R$ in $R^{\prime}$, that is all elements of $R^{\prime}$ that are separable over $R$. The extension $R^{s} \subseteq R^{\prime}$ is then purely inseparable, i.e. some power of the Frobenius has the property that $F^{i}\left(R^{\prime}\right) \subseteq R^{s}$. Applying the functor $H_{I}^{i}\left(\_\right)$yields the diagram

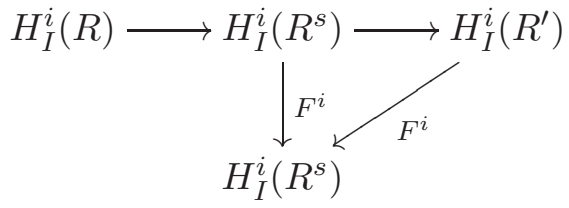

Denoting the image of $\alpha$ in $H_{I}^{i}\left(R^{s}\right)$ by $a$, this element is mapped to zero $H_{I}^{i}\left(R^{\prime}\right)$, hence, under the Frobenius $F^{i}$ it is mapped also to zero in $H_{I}^{i}\left(R^{s}\right)$. But this shows that $F^{i}(a)=0$ 
in $H_{I}^{i}\left(R^{s}\right)$ by the above diagram. Now $\underline{\mathrm{SS} 12}$, Theorem 1.3(1)] states that there is a module finite separable (even Galois with solvable Galois group) extension $R^{s} \subseteq R^{\prime \prime}$ such that $a$ is mapped to zero in $H_{I}^{i}\left(R^{\prime \prime}\right)$. But this means that the finite separable extension $R \subseteq R^{\prime \prime}$ is as required: the image of $\alpha \in H_{I}^{i}(R)$ in $H_{I}^{i}\left(R^{\prime \prime}\right)$ is zero.

The main result of this section, immediately below, is a straightforward application of the method of C. Huneke and G. Lyubeznik in [HL07. Since making this observation, we have been informed that a Matlis dual version of the theorem below has also been obtained by M. Hochster and Y. Yao (in a non-public preprint [HY11]).

Theorem 3.2. Suppose $X$ is an integral $F$-finite scheme.

(a) For all proper dominant maps $\pi: Y \rightarrow X$ with $Y$ integral, the image of the trace map (2.12) contains the parameter test module, i.e.

$$
\tau\left(\omega_{X}\right) \subseteq \operatorname{Image}\left(\mathbf{h}^{\operatorname{dim} Y-\operatorname{dim} X} \mathbf{R} \pi_{*} \omega_{Y} \stackrel{\operatorname{Tr}_{\pi}}{\longrightarrow} \omega_{X}\right) .
$$

(b) There exists a finite separable map $\pi: Y \rightarrow X$ with $Y$ integral such that the image of the trace map equals the parameter test module, i.e.

$$
\tau\left(\omega_{X}\right)=\operatorname{Image}\left(\mathbf{h}^{\operatorname{dim} Y-\operatorname{dim} X} \mathbf{R} \pi_{*} \omega_{Y} \stackrel{\operatorname{Tr}_{\pi}}{\longrightarrow} \omega_{X}\right) .
$$

Remark 3.3. In the above result, it is possible to work with equidimensional reduced (rather than integral) schemes of finite type over a field at the expense of removing "separable" from the conclusion in (b).

An immediate Corollary of this statement is a characterization of the test submodule. Recall that an alteration is a generically finite proper dominant morphism of integral schemes.

Corollary 3.4. Suppose $X$ is an integral F-finite scheme. Then

$$
\tau\left(\omega_{X}\right)=\bigcap_{\pi: Y \longrightarrow X} \text { Image }\left(\mathbf{h}^{\operatorname{dim} Y-\operatorname{dim} X} \mathbf{R} \pi_{*} \omega_{Y} \stackrel{\operatorname{Tr}_{\pi}}{\longrightarrow} \omega_{X}\right) .
$$

where $\pi$ ranges over all of the maps from an integral scheme $Y$ to $X$ that are either:

- (separable) finite dominant maps, or

- (separable) alterations, or

- (separable) proper dominant maps.

Additionally, if $X$ is a variety over a perfect field, then we may allow $\pi$ to range over

- all regular separable alterations to $X$, or

- all separable proper dominant maps with $Y$ regular.

Furthermore, in this case, there always exists a separable regular alteration $\pi: Y \rightarrow X$ such that Image $\left(\pi_{*} \omega_{Y} \stackrel{\operatorname{Tr}_{\pi}}{\longrightarrow} \omega_{X}\right)$ is equal to the parameter test submodule of $X$.

Proof. This follows immediately from Theorem 3.2 and from the existence of regular alterations dJ96.

Proof of Theorem 3.2 (a). The statement follows immediately from Propositions 2.21 and 2.13 as well as the definition of $\tau\left(\omega_{X}\right)$.

The proof of (b) follows closely the strategy of [HL07]; note that a local version of the statement is also related to the result of K. Smith that "plus closure equals tight closure for parameter ideals" SSmi94]. The proof comes down to Noetherian induction once we establish the following lemma. 
Lemma 3.5. Let $R \subseteq S$ be a module finite inclusion of domains and denote the image of the trace map by $J_{S}=$ Image $\left(\omega_{S} \rightarrow \omega_{R}\right)$. If $\tau\left(\omega_{R}\right) \subsetneq J_{S}$, then there is a separable finite extension of domains $S \subseteq S^{\prime}$ such that the support of $J_{S^{\prime}} / \tau\left(\omega_{R}\right)$ is strictly contained in the support of $J_{S} / \tau\left(\omega_{R}\right)$, where $J_{S^{\prime}}=\operatorname{Image}\left(\omega_{S^{\prime}} \longrightarrow \omega_{R}\right)$.

Proof. Choose $\eta \in$ Spec $R$ to be the generic point of a component of the support of $J_{S} / \tau\left(\omega_{R}\right)$ and set $d=\operatorname{dim} R_{\eta}$. By construction $\left(J_{S} / \tau\left(\omega_{R}\right)\right)_{\eta}=J_{S_{\eta}} / \tau\left(\omega_{R_{\eta}}\right)$ has finite length, and hence so also must its Matlis dual

$$
\left(J_{S} / \tau\left(\omega_{R}\right)\right)_{\eta}^{\vee}=\operatorname{Hom}\left(J_{S_{\eta}} / \tau\left(\omega_{R_{\eta}}\right), E\left(R_{\eta} / \eta\right)\right) .
$$

By Matlis duality applied to the sequence $\omega_{S_{\eta}} \rightarrow\left(J_{S_{\eta}} / \tau\left(\omega_{R_{\eta}}\right)\right) \hookrightarrow \omega_{R_{\eta}} / \tau\left(\omega_{R_{\eta}}\right)$ one observes that $\left(J_{S} / \tau\left(\omega_{R}\right)\right)_{\eta}^{\vee} \subseteq H_{\eta}^{d}\left(S_{\eta}\right)$ is identified with the image of the tight closure of zero $0_{H_{\eta}^{d}\left(R_{\eta}\right)}^{*}=\left(\omega_{R_{\eta}} / \tau\left(\omega_{R_{\eta}}\right)\right)^{\vee}$ in $H_{\eta}^{d}\left(S_{\eta}\right)$. By Proposition 2.21 we have for $\Phi_{R}: F_{*} \omega_{R} \rightarrow \omega_{R}$ that $\Phi_{R}\left(J_{S}\right) \subseteq J_{S}$. Hence the dual of $J_{S} / \tau\left(\omega_{R}\right)$ is stable under the action of the Frobenius on $H_{\eta}^{d}\left(S_{\eta}\right)$. i.e. $F\left(\left(J_{S} / \tau\left(\omega_{R}\right)\right)_{\eta}^{\vee}\right) \subseteq\left(J_{S} / \tau\left(\omega_{R}\right)\right)_{\eta}^{\vee}$ (phrased differently: the tight closure of zero is Frobenius stable, hence so is its image). This implies that for any element $\alpha \in\left(J_{S} / \tau\left(\omega_{R}\right)\right)_{\eta}^{\vee}$ the powers $\alpha, \alpha^{p}, \alpha^{p^{2}}, \ldots$ must also lie in the finite length $\left(J_{S_{\eta}} / \tau\left(\omega_{R_{\eta}}\right)\right)^{\vee}$. Applying Lemma 3.1 to $\alpha \in\left(J_{S_{\eta}} / \tau\left(\omega_{R_{\eta}}\right)\right)^{\vee}$ repeatedly (e.g. for a finite set of generators) we obtain a separable integral extension $S_{\eta} \subseteq T$ such that $H_{\eta}^{d}\left(S_{\eta}\right) \rightarrow H_{\eta}^{d}(T)$ maps $\left(J_{S_{\eta}} / \tau\left(\omega_{R_{\eta}}\right)\right)^{\vee}$ to zero. By taking $S^{\prime}$ to be the normalization of $S$ in the total field of fractions of $T$ we see that $T=S_{\eta}^{\prime}$ and that the finite extension $R \subseteq S^{\prime}$ is separable. Translating this back via Matlis duality this means that the map $\omega_{S_{\eta}^{\prime}} \rightarrow \omega_{R_{\eta}}$ sends $J_{S_{\eta}^{\prime}}$ into $\tau\left(\omega_{R_{\eta}}\right)$. In particular $\eta \notin \operatorname{Supp}\left(J_{S^{\prime}} / \tau\left(\omega_{R}\right)\right)$.

Proof of Theorem 3.2 (b). Without loss of generality, we may assume that $X=\operatorname{Spec} R$ is affine. Starting with the identity $R=S_{0}$ we successively produce, using Lemma 3.5, a sequence of separable finite extensions $R=S_{0} \subseteq S_{1} \subseteq S_{2} \subseteq S_{3} \ldots$ such that the support of $J_{S_{i+1}} / \tau\left(\omega_{R}\right)$ is strictly smaller than the support of $J_{S_{i}} / \tau\left(\omega_{R}\right)$ until $J_{S_{i}}=\tau\left(\omega_{R}\right)$ by Noetherian induction.

The following important corollary (which can also be proven directly from the equational lemma without reference to the above results) should be viewed in the context of the definition of pseudo-rationality (see Section 2.4), as well as the Kempf-criterion for rational singularity [KKMSD73, p. 50] in characteristic zero.

Corollary 3.6. For an F-finite Cohen-Macaulay domain $R$ the following are equivalent.

(a) $R$ is $F$-rational, i.e. there is no non-trivial submodule $M \subseteq \omega_{R}$ which is stable under $\Phi_{R}: F_{*} \omega_{R} \rightarrow \omega_{R}$.

(b) For all finite extensions $R \rightarrow S$ (which may be taken to be separable if desired) the induced map $\omega_{S} \rightarrow \omega_{R}$ is surjective.

(c) For all alterations $\pi: Y \rightarrow X=\operatorname{Spec} R$ ( $\pi$ may be taken to be separable and or regular if $R$ is of finite type over a perfect field) the induced map $\pi_{*} \omega_{Y} \rightarrow \omega_{X}$ is surjective.

In fact, utilizing local duality and [HL07] once again to obtain a further finite cover which annihilates the local cohomology modules below the dimension, we obtain the following characterization of $F$-rationality without the Cohen-Macaulay hypothesis.

Corollary 3.7. For an F-finite domain $R$, the following are equivalent:

(a) $R$ is F-rational. 
(b) For each integer $i \in \mathbb{Z}$, and every (separable) finite extension of rings $R \rightarrow S$, the induced map $\mathbf{h}^{i} \omega_{S}^{\cdot} \longrightarrow \mathbf{h}^{i} \omega_{R}^{\cdot}$ is surjective.

(c) For each integer $i \in \mathbb{Z}$ and every (separable regular, if $R$ is of finite type over a perfect field) alteration $\pi: Y \rightarrow X=\operatorname{Spec} R$, the induced map $\mathbf{h}^{i} \mathbf{R} \pi_{*} \omega_{Y}^{\cdot} \rightarrow \mathbf{h}^{i} \omega_{X}^{\bullet}$ is surjective.

Remark 3.8. The main result of the paper [HL07] which inspired our proof is that for a local ring $(R, \mathfrak{m})$ of dimension $d$ that is a homomorphic image of a Gorenstein ring, there is a module finite extension $R \subseteq S$ such that the induced map $H_{\mathfrak{m}}^{i}(R) \rightarrow H_{\mathfrak{m}}^{i}(S)$ is zero for $i<d$. A local dual statement to this is that the induced map on dualizing complexes $\omega_{S}^{*} \rightarrow \omega_{R}^{\cdot}$ is zero on cohomology $\mathbf{h}^{i}\left(\omega_{\dot{S}}^{\bullet}\right) \rightarrow \mathbf{h}^{i}\left(\omega_{R}^{\cdot}\right)$ for $i \neq-\operatorname{dim} R$. What we accomplish here is that we clarify the case $i=-\operatorname{dim} R$. With $d=\operatorname{dim} R$ we just showed that one can also achieve that the image $\mathbf{h}^{-d}\left(\omega_{S}^{\cdot}\right) \rightarrow \mathbf{h}^{-d}\left(\omega_{R}^{\bullet}\right) \cong \omega_{R}$ is the parameter test submodule $\tau\left(\omega_{R}\right)$. Of course, the dual statement thereof is: the tight closure of zero $0_{H_{\mathfrak{m}}^{d}(R)}^{*}$ is mapped to zero under the map $H_{\mathfrak{m}}^{d}(R) \rightarrow H_{\mathfrak{m}}^{d}(S)$. It is exactly this statement, and further generalizations, which are first and independently by M. Hochster and Y. Yao in HY11.

\section{Test ideals Via alterations}

In this section, we explore the behavior of test ideals under proper dominant maps and prove our main theorem in characteristic $p>0$ in full generality. First we show that various images are compatible with the $\Phi$ from Example 2.4, and so they contain the test ideal.

Proposition 4.1. Suppose that $f: Y \rightarrow X$ is a proper dominant generically finite map of normal $F$-finite varieties and that $(X, \Delta)$ is a log- $\mathbb{Q}$-Gorenstein pair. Then the test ideal is contained in the image of the trace map, i.e.

$$
\tau(X ; \Delta) \subseteq \text { Image }\left(f_{*} \omega_{Y}\left(-\left\lfloor f^{*}\left(K_{X}+\Delta\right)\right\rfloor\right) \stackrel{\operatorname{Tr}_{f}}{\longrightarrow} \omega_{X}\left(-\left\lfloor K_{X}+\Delta\right\rfloor\right) \subseteq K(X)\right)
$$

where $\operatorname{Tr}_{f}$ is the map induced by trace as in Proposition 2.8.

Proof. This follows easily by Stein factorization. Factor $f$ as $Y \stackrel{g}{\rightarrow} Z \stackrel{h}{\rightarrow} X$ where $g$ is birational and $h$ is finite. Then it follows from [ST10, Theorem 6.25] (cf. [ST12b, Lemma 4.4(a)]) that

$$
\operatorname{Tr}_{h}\left(h_{*} \tau\left(Z ;-K_{Z}+h^{*}\left(K_{X}+\Delta\right)\right)\right)=\tau(X ; \Delta) .
$$

On the other hand, it follows from the argument that the test ideal is contained in the multiplier ideal (since the test ideal is the unique smallest ideal satisfying a certain property), that

$$
\operatorname{Tr}_{g}\left(g_{*} \omega_{Y}\left(-\left\lfloor f^{*}\left(K_{X}+\Delta\right)\right\rfloor\right)\right) \supseteq \tau\left(Z ;-K_{Z}+h^{*}\left(K_{X}+\Delta\right)\right) .
$$

See [Tak04] or see [ST12a, Theorem 4.17] for a sketch of a simpler version of this argument (or see immediately below for a generalization).

This completes the proof since $\operatorname{Tr}_{h} \circ h_{*} \operatorname{Tr}_{g}=\operatorname{Tr}_{f}$.

We also prove a more general statement whose proof also partially generalizes Proposition 2.21. and which uses heavily the material from the preliminary section on Duality, Section 2.3. The reader who has so far avoided that section, can skip Proposition 4.2 and rely on Proposition 4.1 instead.

Proposition 4.2. Suppose that $f: Y \rightarrow X$ is a proper dominant map of normal integral $F$-finite schemes and that $(X, \Delta)$ is a $\log -\mathbb{Q}$-Gorenstein pair. Then the test ideal is contained 
in the image of the trace map, i.e.

$\tau(X ; \Delta) \subseteq$ Image $\left(\mathbf{h}^{\operatorname{dim} Y-\operatorname{dim} X} \mathbf{R} f_{*} \omega_{Y}\left(-\left\lfloor f^{*}\left(K_{X}+\Delta\right)\right\rfloor\right) \stackrel{\operatorname{Tr}_{f}}{\longrightarrow} \omega_{X}\left(-\left\lfloor K_{X}+\Delta\right\rfloor\right) \subseteq K(X)\right)$ where $\operatorname{Tr}_{f}$ is the map induced by trace as in Proposition 2.18. Similarly, if additionally $f^{*}\left(K_{X}+\Delta\right)$ is a Cartier divisor, then

$$
\tau(X ; \Delta) \subseteq \operatorname{Image}\left(\mathbf{h}^{-\operatorname{dim} X} \mathbf{R} f_{*} \omega_{Y}\left(f^{*}\left(K_{X}+\Delta\right)\right) \stackrel{\operatorname{Tr}_{f} \bullet}{\longrightarrow} \omega_{X}\left(-\left\lfloor K_{X}+\Delta\right\rfloor\right) \subseteq K(X)\right)
$$

where again $\operatorname{Tr}_{f} \cdot$ is the map induced by trace as in Proposition 2.18.

Proof. The statement is local (if it fails to hold, then it fails to hold locally), so we assume that $X$ is the spectrum of a local ring. Without loss of generality, as in Definition 2.37, we may assume that $\Delta \geq 0$. Let $n=\operatorname{dim} Y-\operatorname{dim} X$.

Fix an $\mathscr{O}_{X}$-linear map $\phi: F_{*}^{e} \mathscr{O}_{X} \rightarrow \mathscr{O}_{X}$ such that $\Delta_{\phi} \geq \Delta$, where $\Delta_{\phi}$ is defined as in Sch09, Theorem 3.11, 3.13] or [ST12a, Subsection 4.4]. Recall that $\left(p^{e}-1\right)\left(K_{X}+\Delta_{\phi}\right) \sim 0$, so in particular we may write $\left(p^{e}-1\right)\left(K_{X}+\Delta\right)=\operatorname{div} c$ for some $c \in K(X)$. For the first statement, it is sufficient to show that

$$
\phi\left(F_{*}^{e} \operatorname{Tr}_{f}\left(\mathbf{h}^{n} \mathbf{R} f_{*} \omega_{Y}\left(-\left\lfloor f^{*}\left(K_{X}+\Delta\right)\right\rfloor\right)\right)\right) \subseteq \operatorname{Tr}_{f}\left(\mathbf{h}^{n} \mathbf{R} f_{*} \omega_{Y}\left(-\left\lfloor f^{*}\left(K_{X}+\Delta\right)\right\rfloor\right)\right)
$$

and by Lemma 2.20, we may assume $\phi\left(\_\right)=\Phi_{X}^{e}\left(F_{*}^{e} c \cdot{ }_{-}\right)$. Now we have

$$
\phi\left(F_{*}^{e} \operatorname{Tr}_{f}\left(\_\right)\right)=\Phi_{X}^{e}\left(\left(F_{*}^{e} c\right) \cdot F_{*}^{e} \operatorname{Tr}_{f}\left(Z_{)}\right)=\Phi_{X}^{e}\left(F_{*}^{e} \operatorname{Tr}_{f}\left(c \cdot Z_{-}\right)\right)=\operatorname{Tr}_{f}\left(\mathbf{h}^{n} \mathbf{R} f_{*} \Phi_{Y}^{e}\left(F_{*}^{e} c \cdot Z_{-}\right)\right)\right.
$$

where we have used that $\operatorname{Tr}_{f}$ is $\mathscr{O}_{X}$-linear, and that $\Phi_{X}^{e}\left(F_{*}^{e} \operatorname{Tr}_{f}\left(\_\right)\right)=\operatorname{Tr}_{f}\left(\mathbf{h}^{n} \mathbf{R} f_{*} \Phi_{Y}^{e}\left(F_{*}^{e}-\right)\right)$ as shown in (2.23). Thus, it is enough to show

$$
\Phi_{Y}^{e}\left(F_{*}^{e}\left(c \cdot \omega_{Y}\left(-\left\lfloor f^{*}\left(K_{X}+\Delta\right)\right\rfloor\right)\right)\right) \subseteq \omega_{Y}\left(-\left\lfloor f^{*}\left(K_{X}+\Delta\right)\right\rfloor\right) .
$$

Since

$$
-\left\lfloor f^{*}\left(K_{X}+\Delta\right)\right\rfloor+\left(1-p^{e}\right) f^{*}\left(K_{X}+\Delta_{\phi}\right)=-\left\lfloor f^{*}\left(p^{e} K_{X}+\left(p^{e}-1\right) \Delta_{\phi}+\Delta\right)\right\rfloor
$$

and $\Delta_{\phi} \geq \Delta$, we have

$$
c \cdot \omega_{Y}\left(-\left\lfloor f^{*}\left(K_{X}+\Delta\right)\right\rfloor\right) \subseteq \omega_{Y}\left(-\left\lfloor p^{e} f^{*}\left(K_{X}+\Delta\right)\right\rfloor\right)=\omega_{Y}\left(-\left\lfloor\left(F^{e}\right)^{*} f^{*}\left(K_{X}+\Delta\right)\right\rfloor\right) .
$$

But then, according to Proposition 2.18, we have

$$
\Phi_{Y}^{e}\left(F_{*}^{e} \omega_{Y}\left(-\left\lfloor\left(F^{e}\right)^{*} f^{*}\left(K_{X}+\Delta\right)\right\rfloor\right)\right) \subseteq \omega_{Y}\left(-\left\lfloor f^{*}\left(K_{X}+\Delta\right)\right\rfloor\right)
$$

which completes the proof of the first statement. For the second statement, simply notice that Image $\left(\operatorname{Tr}_{f} \bullet\right) \supseteq \operatorname{Image}\left(\operatorname{Tr}_{f}\right)$.

Lemma 4.3. Suppose that $(X=\operatorname{Spec} R, \Gamma)$ is a pair such that $\Gamma=t \operatorname{div}(g)$ for some $g \in R$ and $t \in \mathbb{Q}_{\geq 0}$. Fix $c \in R$ such that $R_{c}$ is regular and that $\operatorname{Supp}(\Gamma)=V(g) \subseteq V(c)$. Then there exists a power of $c^{N}$ of $c$ such that

$$
\tau\left(\omega_{X} ; \Gamma\right)=\sum_{e \geq 0} \Phi^{e}\left(F_{*}^{e} c^{N} g^{\left\lceil t\left(p^{e}-1\right)\right\rceil} \omega_{R}\right)
$$

where $\Phi_{R}^{e}: F_{*}^{e} \omega_{R} \rightarrow \omega_{R}$ denotes the trace of the e-iterated Frobenius, see Section 2.3.

Proof. By the usual theory of test elements ( $c f$. [Sch11, proof of Theorem 3.18]), we have for some power $c^{n}$ of $c$ that

$$
\tau\left(\omega_{X} ; \Gamma\right)=\sum_{e \geq 0} \sum_{\phi} \phi\left(F_{*}^{e} c^{n} \omega_{R}\right)
$$


where the inner sum ranges over all $\phi \in \operatorname{Hom}_{R}\left(F_{*}^{e} \omega_{R}\left(\left\lceil\left(p^{e}-1\right) \Gamma\right\rceil\right), \omega_{R}\right)$. Furthermore, note it is harmless to replace $n$ by any larger integer $n+k$. The reason the statement does not follow immediately is that, as $\operatorname{div}(g)$ may not be reduced, we may have $\left\lceil t\left(p^{e}-1\right)\right\rceil \operatorname{div}(g) \geq$ $\left\lceil t\left(p^{e}-1\right) \operatorname{div}(g)\right\rceil$. Choose $k$ such that $\operatorname{div}\left(c^{k}\right)+\left(p^{e}-1\right) \Gamma \geq \operatorname{div}\left(g^{\left\lceil t\left(p^{e}-1\right)\right\rceil}\right)$ for all $e \geq 0$, and set $N=n+k$.

Now, the map $\psi\left(\_\right)=\Phi_{R}^{e}\left(g^{\left\lceil t\left(p^{e}-1\right)\right\rceil} \cdot \_\right) \in \operatorname{Hom}_{R}\left(F_{*}^{e} \omega_{R}\left(\left\lceil\left(p^{e}-1\right) \Gamma\right\rceil\right), \omega_{R}\right)$ appears in the sum above. This implies the containment $\supseteq$ for our desired equality. Furthermore, for any $\phi \in \operatorname{Hom}_{R}\left(F_{*}^{e} \omega_{R}\left(\left\lceil\left(p^{e}-1\right) \Gamma\right\rceil\right), \omega_{R}\right)$, it is clear then that $\phi\left(F_{*}^{e} c^{N} \omega_{R}\right) \subseteq$ $F_{*}^{e} \Phi^{e}\left(F_{*}^{e} c^{n} g^{\left\lceil\left(p^{e}-1\right)\right\rceil} \omega_{R}\right)$. Thus, we have the containment $\subseteq$ as well.

We now describe the transformation rule for the parameter test module under finite maps.

Proposition 4.4 (cf. [ST10]). Given a finite map $f: Y \rightarrow X$ of normal F-finite integral schemes and a $\mathbb{Q}$-Cartier $\mathbb{Q}$-divisor $\Gamma$ on $X$, we have

$$
\operatorname{Tr}_{f}\left(f_{*} \tau\left(\omega_{Y} ; f^{*} \Gamma\right)\right)=\tau\left(\omega_{X} ; \Gamma\right) .
$$

Proof. Without loss of generality, we may assume that $X=\operatorname{Spec} R$ and $Y=\operatorname{Spec} S$ are affine and that $\Gamma=t \operatorname{div}(g)$ for some $g \in R$.

Let $\Phi_{R}^{e}: F_{*}^{e} \omega_{R} \rightarrow \omega_{R}$ and $\Phi_{S}^{e}: F_{*}^{e} \omega_{S} \rightarrow \omega_{S}$ be the corresponding traces of Frobenius and set $J_{S}=\operatorname{Image}\left(\operatorname{Tr}_{f}: f_{*} \omega_{S} \rightarrow \omega_{R}\right) \subseteq \omega_{R}$. Now, Lemma 4.3 above implies that there exists an element $c \in R$ such that both

$$
\begin{gathered}
\tau\left(\omega_{R} ; \Gamma\right)=\sum_{e \geq 0} \Phi_{R}^{e}\left(F_{*}^{e} c g^{\left\lceil t\left(p^{e}-1\right)\right\rceil} J_{S}\right) \\
\tau\left(\omega_{S} ; f^{*} \Gamma\right)=\sum_{e \geq 0} \Phi_{S}^{e}\left(F_{*}^{e} c g^{\left\lceil t\left(p^{e}-1\right)\right\rceil} \omega_{S}\right) .
\end{gathered}
$$

The idea for the remainder of the proof is to apply $\operatorname{Tr}_{f}\left(\_\right)$to $\tau\left(\omega_{S}, f^{*} \Gamma\right)$, noting that $\operatorname{Tr}_{f}\left(f_{*} \Phi_{S}^{e}(\ldots)\right)=\Phi_{R}^{e}\left(F_{*}^{e} \operatorname{Tr}_{f}\left(\_\right)\right)$since trace is compatible with composition (shown precisely in (2.23) $)$. Therefore,

$$
\begin{aligned}
\operatorname{Tr}_{f}\left(f_{*} \tau\left(\omega_{S} ; f^{*} \Gamma\right)\right) & =\operatorname{Tr}_{f}\left(f_{*}\left(\sum_{e \geq 0} \Phi_{S}^{e}\left(F_{*}^{e} c g^{\left\lceil t\left(p^{e}-1\right)\right\rceil} \omega_{S}\right)\right)\right) \\
& =\sum_{e \geq 0} \operatorname{Tr}_{f}\left(f_{*} \Phi_{S}^{e}\left(F_{*}^{e} c g^{\left\lceil t\left(p^{e}-1\right)\right\rceil} f_{*} \omega_{S}\right)\right) \\
& =\sum_{e \geq 0} \Phi_{R}^{e}\left(F_{*}^{e} \operatorname{Tr}_{f}\left(c g^{\left\lceil t\left(p^{e}-1\right)\right\rceil} f_{*} \omega_{S}\right)\right) \\
& =\sum_{e \geq 0} \Phi_{R}^{e}\left(F_{*}^{e} c g^{\left\lceil t\left(p^{e}-1\right)\right\rceil} J_{S}\right) \\
& =\tau\left(\omega_{R} ; \Gamma\right)
\end{aligned}
$$

which completes the proof.

To reduce the main theorem of this paper to Theorem 3.2, we need a variant of the cyclic covering construction, $c f$. [TW92] or [KM98, Section 2.4].

Lemma 4.5. Suppose that $X$ is a normal integral scheme and $\Gamma$ is a $\mathbb{Q}$-Cartier $\mathbb{Q}$-divisor on $X$. Then there exists a finite separable $\operatorname{map} g: W \rightarrow X$ from a normal integral scheme $W$ such that $g^{*} \Gamma$ is a Cartier divisor. 
Proof. We may assume that $X=\operatorname{Spec} R$ is affine and that $n \Gamma=\operatorname{div}_{X}(f)$ for some non-zero non-unit $f \in R$. We view $f \in K=K(R)$ and suppose that $\alpha$ is a root of the polynomial $x^{n}+f x+f$ in some separable finite field extension $L$ of $K$. Let $S$ be the normalization of $R$ inside $L$ so that we have a module-finite inclusion $R \subseteq S$. Set $\pi: Y=\operatorname{Spec} S \rightarrow \operatorname{Spec} R=$ $X$. Further observe that $S$ contains $\alpha$ since $\alpha$ is integral over $R$. Since $\alpha^{n}=-f(\alpha+1)$ we have $\alpha, \alpha+1 \in \sqrt{\langle\alpha+1\rangle}$, so that $\alpha+1$ is a unit. Thus, $n \operatorname{div}_{Y}(\alpha)=\operatorname{div}_{Y}(f)=\pi^{*} n \Gamma$, and so $\pi^{*} \Gamma=\operatorname{div}_{Y}(\alpha)$ is Cartier as desired.

Theorem 4.6. Given a normal integral $F$-finite scheme $X$ with a $\mathbb{Q}$-divisor $\Delta$ such that $K_{X}+\Delta$ is $\mathbb{Q}$-Cartier, there exists a finite separable map $f: Y \rightarrow X$ from a normal $F$-finite integral scheme $Y$ such that

$$
\tau(X ; \Delta)=\operatorname{Image}\left(f_{*} \omega_{Y}\left(-\left\lfloor f^{*}\left(K_{X}+\Delta\right)\right\rfloor\right) \stackrel{\operatorname{Tr}_{f}}{\longrightarrow} K(X)\right) .
$$

Alternatively, if $X$ is of finite type over a F-finite (respectively perfect) field, one may take $f: Y \rightarrow X$ to be a regular (respectively separable) alteration.

Before proving this theorem, we state several corollaries.

Corollary 4.8. Assume that $X$ is a normal variety over an F-finite field $k$. If $(X, \Delta)$ is a $\log -\mathbb{Q}$-Gorenstein pair, then using the images of the trace map as in Proposition 2.18, we have

$$
\tau(X ; \Delta)=\bigcap_{f: Y \longrightarrow X} \operatorname{Image}\left(\mathbf{h}^{\operatorname{dim} Y-\operatorname{dim} X} \mathbf{R} f_{*} \omega_{Y}\left(-\left\lfloor f^{*}\left(K_{X}+\Delta\right)\right\rfloor\right) \stackrel{\operatorname{Tr}_{f}}{\longrightarrow} K(X)\right)
$$

where $f: Y \rightarrow X$ ranges over all maps from a normal variety $Y$ that are either:

(a) finite dominant maps

(b) finite separable dominant maps

(c) alterations (i.e. generically finite proper dominant maps)

(d) regular alterations

(e) proper dominant maps

(f) proper dominant maps from regular schemes

or, if additionally $k$ is perfect,

(g) regular separable alterations.

Furthermore, in all cases the intersection stabilizes (i.e. is equal to one of its members).

Corollary 4.10. Assume $X$ is a normal integral $F$-finite scheme with a $\mathbb{Q}$-divisor $\Delta$ such that $K_{X}+\Delta$ is $\mathbb{Q}$-Cartier. Then using the images of the trace map as in Proposition 2.18. we have

$$
\tau(X ; \Delta)=\bigcap_{f: Y \rightarrow X} \text { Image }\left(\mathbf{h}^{-\operatorname{dim} X} \mathbf{R} f_{*} \omega_{Y}\left(-\left\lfloor f^{*}\left(K_{X}+\Delta\right)\right\rfloor\right) \stackrel{\operatorname{Tr}_{f} \bullet}{\longrightarrow} K(X)\right)
$$

where the intersection runs over all proper dominant maps $f: Y \rightarrow X$ from a normal integral scheme $Y$ such that $f^{*}\left(K_{X}+\Delta\right)$ is Cartier. Furthermore, once again, the intersection stabilizes.

Proof of Theorem 4.6. We make use the identification of $\tau(X ; \Delta)=\tau\left(\omega_{X} ; K_{X}+\Delta\right)$ from Lemma 2.39 and will prove the statement for the latter. For (4.7), first take a separable finite map $f^{\prime}: X^{\prime} \rightarrow X$ such that $\Gamma:=f^{\prime *}\left(K_{X}+\Delta\right)$ is Cartier by Lemma 4.5, By Proposition 4.4,

$$
\operatorname{Tr}_{f^{\prime}}\left(f_{*}^{\prime} \tau\left(\omega_{X^{\prime}} ; \Gamma\right)\right)=\tau\left(\omega_{X} ; K_{X}+\Delta\right)=\tau(X ; \Delta) .
$$


Now, using Theorem 3.2 we may fix a separable finite map $h: Y \rightarrow X^{\prime}$ such that we have $\tau\left(\omega_{X^{\prime}}\right)=$ Image $\left(\operatorname{Tr}_{h}: h_{*} \omega_{Y} \rightarrow \omega_{X^{\prime}}\right)$. The projection formula then gives the equality $\tau\left(\omega_{X^{\prime}} ; \Gamma\right)=\operatorname{Image}\left(\operatorname{Tr}_{h}\left(h_{*} \omega_{Y}\left(-h^{*} \Gamma\right) \rightarrow \omega_{X^{\prime}}\right)\right.$, and (4.7) now follows after applying $\operatorname{Tr}_{f^{\prime}}$.

For the remaining statement when $X$, if we are given a composition of alterations $f \circ g$ : $Z \stackrel{g}{\rightarrow} Y \stackrel{f}{\rightarrow} X$, it follows that

$$
\begin{aligned}
& \operatorname{Image}\left(f_{*} g_{*} \omega_{Z}\left(-\left\lfloor g^{*} f^{*}\left(K_{X}+\Delta\right)\right\rfloor\right) \stackrel{\operatorname{Tr}_{f \circ g}}{\longrightarrow} K(X)\right) \\
& \subseteq \operatorname{Image}\left(f_{*} \omega_{Y}\left(-\left\lfloor f^{*}\left(K_{X}+\Delta\right)\right\rfloor\right) \stackrel{\operatorname{Tr}_{f}}{\longrightarrow} K(X)\right) .
\end{aligned}
$$

Thus, the second statement immediately follows from the first by taking a further regular (separable) alteration using [J96, Theorem 4.1].

Proof of Corollary 4.8. For equation (4.9), we have the containment $\tau(X ; \Delta) \subseteq$ from Proposition 4.1 or Proposition 4.2. The result then follows from equation (4.7).

Proof of Corollary 4.10. Finally, for equation (4.11) we still have the containment $\tau(X ; \Delta) \subseteq$ from Proposition 4.2. On the other hand, if $Y$ has the same dimension as $X$, then it is readily seen from the spectral sequence argument used in the proof of Proposition 2.13 that

$\operatorname{Tr}_{f} \cdot\left(\mathbf{h}^{-\operatorname{dim} X} \mathbf{R} f_{*} \omega_{Y}\left(-\left\lfloor f^{*}\left(K_{X}+\Delta\right)\right\rfloor\right)\right)=\operatorname{Tr}_{f}\left(\mathbf{h}^{\operatorname{dim} Y-\operatorname{dim} X} \mathbf{R} f_{*} \omega_{Y}\left(-\left\lfloor f^{*}\left(K_{X}+\Delta\right)\right\rfloor\right)\right)$.

so that equality holds in (4.11) for $f: Y \rightarrow X$ as in Theorem 4.6.

Remark 4.12. If $R$ is an $F$-finite $\mathbb{Q}$-Gorenstein splinter ring (i.e. any module-finite extension $R \subseteq S$ splits as a map of $R$-modules), then Corollary 4.8 above gives that $\tau(X)=\mathscr{O}_{X}$, implying that $R$ is strongly $F$-regular (since by $\tau(X)$ we always mean the big test ideal). This recovers the main result of [Sin99.

\section{NADEL-TYPE VANISHING UP TO FINITE MAPS}

Among the most sorely missed tools in positive characteristic birational algebraic geometry (in comparison to characteristic zero) are powerful cohomology vanishing theorems. Strong additional assumptions (e.g. lifting to the second Witt vectors) are required to recover the most basic version of Kodaira vanishing, and even under similar assumptions the most powerful variants (e.g. Kawamata-Viehweg or Nadel-type vanishing) cannot be proven. By applying the results and ideas of B. Bhatt's dissertation (see also [Bha12]), we derive here variants of Nadel-type vanishing theorems. These are strictly weaker than what one would hope for as we only obtain the desired vanishing after a finite covering. Notably however, we need not require a W2 lifting hypothesis.

Before continuing, we recall the following well known Lemma.

Lemma 5.1 (cf. Bha10]). Suppose that $X$ is a Noetherian scheme and that we have a map of objects $f: A^{\bullet} \rightarrow B^{\bullet}$ within $D_{\text {coh }}^{\geq 0}(X)$. Then $f$ factors through $\mathbf{h}^{0} B^{\bullet}$ if and only if $\boldsymbol{\tau}_{>0}(f)$ is the zero map.

Proof. Certainly if $f$ factors through $\mathbf{h}^{0} B^{\bullet}$, then $\boldsymbol{\tau}_{>0}(f)=0$ since $\boldsymbol{\tau}_{>0}\left(\mathbf{h}^{0} B^{\bullet}\right)=0$. For the converse direction, suppose $\boldsymbol{\tau}_{>0}(f)=0$. Consider the diagram

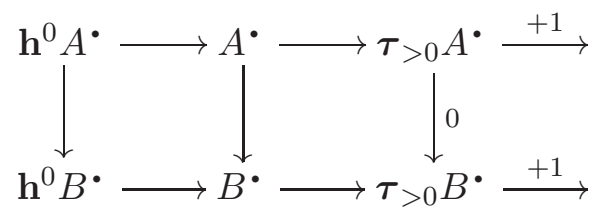


Since $A^{\bullet} \rightarrow B^{\bullet} \rightarrow \boldsymbol{\tau}_{>0} B^{\bullet}$ is zero, we also have the following diagram of objects in $D_{\text {coh }}^{\geq 0}(X)$

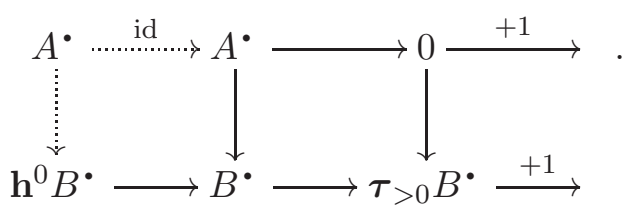

We begin with a lemma which can be viewed as a kind of "Grauert-Riemenschneider vanishing GR70 up to finite maps." Using the notation from below, note that in the special case where $W$ is smooth (or a tame quotient singularity) it has been shown only recently by A. Chatzistamatiou and K. Rülling [CR11] that $\mathbf{h}^{i} \mathbf{R} \pi_{*} \omega_{X}$ is zero for $i>0$.

Lemma 5.2. Suppose that $\pi: X \rightarrow W$ is an alteration between integral schemes of characteristic $p>0$. Then there exists a finite map $g: U \rightarrow X$ such that the trace map

$$
\boldsymbol{\tau}_{>-\operatorname{dim} X} \mathbf{R}(\pi \circ g)_{*} \omega_{\dot{U}}^{\bullet} \rightarrow \boldsymbol{\tau}_{>-\operatorname{dim} X} \mathbf{R} \pi_{*} \omega_{W}^{\bullet}
$$

is zero and furthermore that the trace map

$$
\boldsymbol{\tau}_{>0} \mathbf{R}(\pi \circ g)_{*} \omega_{U} \longrightarrow \boldsymbol{\tau}_{>0} \mathbf{R} \pi_{*} \omega_{W}
$$

is zero. As a consequence $\mathbf{h}^{i} \mathbf{R}(\pi \circ g)_{*} \omega_{U} \longrightarrow \mathbf{h}^{i} \mathbf{R} \pi_{*} \omega_{X}$ is zero for all $i>0$.

Proof. It is harmless to assume that $\pi$ is birational (simply take the normalization of $W$ inside the fraction field of $X$ ) and also that $W$ is affine.

First, choose a finite cover $a: X^{\prime} \rightarrow X$ such that $a: \omega_{X^{\prime}}^{\cdot} \rightarrow \omega_{X}^{\cdot}$ factors through $\omega_{X}[\operatorname{dim} X]$ by [Bha10, Proposition 5.4.2]. Set $X^{\prime} \rightarrow W^{\prime} \rightarrow W$ to be the Stein factorization of $\pi \circ a$ (thus $\ell: W^{\prime} \rightarrow W$ is finite). By [Bha10, Theorem 5.0.1], there exists a finite cover $b: \bar{X} \rightarrow X^{\prime}$ from a normal $\bar{X}$ such that $\mathbf{R}(\pi \circ a)_{*} \mathscr{O}_{X^{\prime}} \rightarrow \mathbf{R}(\pi \circ a \circ b)_{*} \mathscr{O}_{\bar{X}}$ factors through $(\pi \circ a \circ b)_{*} \mathscr{O}_{\bar{X}}$. Set $\bar{X} \rightarrow \bar{W}$ to be the Stein-factorization of $\pi \circ a \circ b$ (thus $m: \bar{W} \rightarrow W^{\prime}$ is finite and $\left.(\pi \circ a \circ b)_{*} \mathscr{O}_{\bar{X}}=(l \circ m)_{*} \mathscr{O}_{\bar{W}}\right)$. Choose a further cover $n: \widetilde{W} \rightarrow \bar{W}$ such that $\boldsymbol{\tau}_{>-\operatorname{dim} X}\left(n_{*} \omega_{\widetilde{W}}\right) \rightarrow \boldsymbol{\tau}_{>-\operatorname{dim} X}\left(\omega_{\bar{W}}^{\bullet}\right)$ is zero by [Bha10, Proposition 5.4.2]. By making $\widetilde{W}$ larger if necessary, we additionally assume that $c: \widetilde{X} \rightarrow \bar{X}$, the normalization of $\bar{X}$ in the fraction field of $\widetilde{W}$, satisfies the condition that $\mathbf{R}(\pi \circ a \circ b)_{*} \mathscr{O}_{\bar{X}} \rightarrow \mathbf{R}(\pi \circ a \circ b \circ c)_{*} \mathscr{O}_{\widetilde{X}}$ factors through $(\pi \circ a \circ b \circ c)_{*} \mathscr{O}_{\widetilde{X}} \cong(l \circ m \circ n)_{*} \mathscr{O}_{\widetilde{W}}$ again using [Bha10, Theorem 5.0.1].

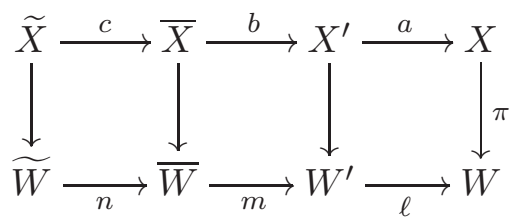

Putting this all together, we have the following factorization of $\mathbf{R} \pi_{*} \mathscr{O}_{X} \rightarrow \mathbf{R} \pi_{*} \mathscr{O}_{\widetilde{X}}$ :

$$
\begin{aligned}
\mathbf{R} \pi_{*} \mathscr{O}_{X} \rightarrow \mathbf{R}(\pi \circ a)_{*} & \mathscr{O}_{X^{\prime}} \rightarrow(\pi \circ a \circ b)_{*} \mathscr{O}_{\bar{X}} \\
& \rightarrow \mathbf{R}(\pi \circ a \circ b)_{*} \mathscr{O}_{\bar{X}} \rightarrow(\pi \circ a \circ b \circ c)_{*} \mathscr{O}_{\widetilde{X}} \rightarrow \mathbf{R}(\pi \circ a \circ b \circ c)_{*} \mathscr{O}_{\tilde{X}}
\end{aligned}
$$

We now note that the term $\mathbf{R}(\pi \circ a \circ b)_{*} \mathscr{O}_{\bar{X}}$ in the factorization can be removed yielding:

$$
\mathbf{R} \pi_{*} \mathscr{O}_{X} \rightarrow \mathbf{R}(\pi \circ a)_{*} \mathscr{O}_{X^{\prime}} \rightarrow(\pi \circ a \circ b)_{*} \mathscr{O}_{\bar{X}} \rightarrow(\pi \circ a \circ b \circ c)_{*} \mathscr{O}_{\widetilde{X}} \rightarrow \mathbf{R}(\pi \circ a \circ b \circ c)_{*} \mathscr{O}_{\widetilde{X}}
$$

which by factoring along the lower part of the above diagram yields

$$
\mathbf{R} \pi_{*} \mathscr{O}_{X} \rightarrow \mathbf{R}(\pi \circ a)_{*} \mathscr{O}_{X^{\prime}} \rightarrow(\ell \circ m)_{*} \mathscr{O}_{\bar{W}} \rightarrow(\ell \circ m \circ n)_{*} \mathscr{O}_{\widetilde{W}} \rightarrow \mathbf{R}(\pi \circ a \circ b \circ c)_{*} \mathscr{O}_{\widetilde{X}}
$$


Now we apply the functor $\mathbf{R} \mathscr{H} o m_{X}\left(\ldots, \omega_{W}^{\bullet}\right)$ to this factorization and obtain:

$$
\mathbf{R} \pi_{*} \omega_{\dot{X}}^{\cdot} \leftarrow \mathbf{R}(\pi \circ a)_{*} \omega_{X^{\prime}} \leftarrow(\ell \circ m)_{*} \omega_{\bar{W}}^{\cdot} \stackrel{\alpha}{\longleftarrow}(\ell \circ m \circ n)_{*} \omega_{\widetilde{W}}^{\cdot} \leftarrow \mathbf{R}(\pi \circ a \circ b \circ c)_{*} \omega_{\tilde{X}}^{\bullet}
$$

Note we don't need $\mathbf{R}$ on $\ell, m$ and $n$ since they are finite. Setting $U=\widetilde{X}$, the first statement of the theorem, (5.3) now follows since $\boldsymbol{\tau}_{>-\operatorname{dim} W}(\alpha)=0$ based on our choice of $n$. However,

$$
\mathbf{R} \pi_{*} \omega_{X}^{\cdot} \longleftarrow \mathbf{R}(\pi \circ a)_{*} \omega_{X^{\prime}}^{\cdot}
$$

factors through $\mathbf{R} \pi_{*} \omega_{X}[\operatorname{dim} X]$. Precomposing with the natural map $\mathbf{R}(\pi \circ a \circ b \circ c)_{*} \omega_{\tilde{X}} \leftarrow$ $\mathbf{R}(\pi \circ a \circ b \circ c)_{*} \omega_{\tilde{X}}[\operatorname{dim} X]$ and taking cohomology yields (5.4).

Theorem 5.5. Suppose that $\pi: X \rightarrow S$ is a proper morphism of F-finite integral schemes of characteristic $p>0$ with $X$ normal. Further suppose that $L$ is a Cartier divisor on $X$ and that $\Delta$ is a $\mathbb{Q}$-divisor on $X$ such that $L-\left(K_{X}+\Delta\right)$ is a $\pi$-big and $\pi$-semi-ample $\mathbb{Q}$-Cartier $\mathbb{Q}$-divisor on $X$. Then there exists a finite surjective map $f: Y \rightarrow X$ from a normal integral $F$-finite scheme $Y$ such that:

(a) The natural trace map

$$
f_{*} \mathscr{O}_{Y}\left(\left\lceil K_{Y}+f^{*}\left(L-\left(K_{X}+\Delta\right)\right)\right\rceil\right) \rightarrow \mathscr{O}_{X}\left(\left\lceil K_{X}+L-\left(K_{X}+\Delta\right)\right\rceil\right)
$$

has image $\tau(X ; \Delta) \otimes \mathscr{O}_{X}(L)$.

(b) The induced map on cohomology

$$
\mathbf{h}^{i} \mathbf{R}(\pi \circ f)_{*} \mathscr{O}_{Y}\left(\left\lceil K_{Y}+f^{*}\left(L-\left(K_{X}+\Delta\right)\right)\right\rceil\right) \rightarrow \mathbf{h}^{i} \mathbf{R} \pi_{*} \tau(X ; \Delta) \otimes \mathscr{O}_{X}(L)
$$

is zero for all $i>0$.

Proof (cf. Bha10]). Certainly by Theorem 4.6 we can assume that (a) holds for some surjective finite map $f^{\prime}: Y^{\prime} \rightarrow X$ (and every further finite map). On $Y^{\prime}$, we may also assume that $f^{\prime *} \Delta$ is integral and $f^{\prime *}\left(K_{X}+\Delta\right)$ is Cartier, and we may further assume that $\mathscr{O}_{Y^{\prime}}\left(f^{\prime *}\left(L-\left(K_{X}+\Delta\right)\right)\right)$ is the pull-back of a line bundle $\mathscr{L}$ via some map $\pi: Y^{\prime} \rightarrow W$ over $S$ such that $\mathscr{L}$ is ample over $S$. Note, $f^{\prime *}\left(L-\left(K_{X}+\Delta\right)\right)$ is still big so we may assume that $W$ has the same dimension as $Y^{\prime}$ (and thus also the same dimension as $X$ ).

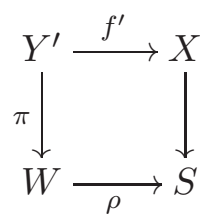

Since we only need now to prove (b), replacing $X$ by $Y^{\prime}$ we may assume that $K_{X}+\Delta$ is Cartier and that $\mathscr{O}_{X}\left(L-\left(K_{X}+\Delta\right)\right)$ is the pull-back of some relatively ample line bundle $\mathscr{L}$ via an alteration $g: X \rightarrow W$ over $S$ with structural map $\rho: W \rightarrow S$.

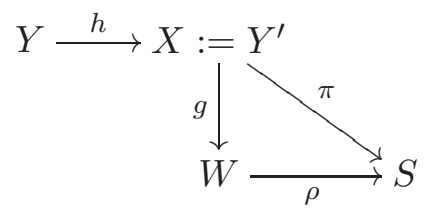

By Lemma 5.2, there exists a finite cover $h: Y \rightarrow X$ such that $\mathbf{R}(g \circ h)_{*} \omega_{Y} \rightarrow \mathbf{R} g_{*} \omega_{X}$ factors through $g_{*} \omega_{X}$. Choose $n_{0}>0$ such that $\mathbf{h}^{i} \mathbf{R} \rho_{*}\left(g_{*} \omega_{X} \otimes \mathscr{L}^{n}\right)=0$ for all $i>0$ and all $n>n_{0}$ (by Serre vanishing). Also choose an integer $e>0$ such that $p^{e}>n_{0}$. Consider 
now $Y \stackrel{h}{\longrightarrow} X \stackrel{g}{\longrightarrow} W \stackrel{F^{e}}{\longrightarrow} W \stackrel{\rho}{\longrightarrow} S$ which can also be expressed as $Y \stackrel{h}{\longrightarrow} X \stackrel{g}{\longrightarrow}$ $W \stackrel{\rho}{\longrightarrow} S \stackrel{F^{e}}{\longrightarrow} S$ and $Y \stackrel{h}{\longrightarrow} X \stackrel{g}{\longrightarrow} W \stackrel{F^{e}}{\longrightarrow} W \stackrel{\rho}{\longrightarrow} S$. Finally consider the factorization:

$$
\begin{aligned}
\mathbf{h}^{i} \mathbf{R}\left(\rho \circ F^{e} \circ g \circ h\right)_{*} & \left(\omega_{Y} \otimes h^{*} g^{*}\left(F^{e}\right)^{*} \mathscr{L}\right)=\mathbf{h}^{i} \mathbf{R}\left(\rho \circ F^{e}\right)_{*}\left(\mathscr{L}^{p^{e}} \otimes \mathbf{R}(g \circ h)_{*} \omega_{Y}\right) \\
& \rightarrow F_{*}^{e} \mathbf{h}^{i}\left(\mathbf{R}(\rho)_{*}\left(\mathscr{L}^{p^{e}} \otimes g_{*} \omega_{X}\right)\right) \\
& \rightarrow F_{*}^{e} \mathbf{h}^{i}\left(\mathbf{R}(\rho)_{*}\left(\mathscr{L}^{p^{e}} \otimes \mathbf{R} g_{*} \omega_{X}\right)\right)=\mathbf{h}^{i}\left(\left(\mathbf{R} \rho_{*}\right) F_{*}^{e}\left(\mathscr{L}^{p^{e}} \otimes \mathbf{R} g_{*} \omega_{X}\right)\right) \\
& \rightarrow \mathbf{h}^{i}\left(\left(\mathbf{R} \rho_{*}\right)\left(\mathscr{L} \otimes \mathbf{R} g_{*} F_{*}^{e} \omega_{X}\right)\right) \\
& \rightarrow \mathbf{h}^{i}\left(\left(\mathbf{R} \rho_{*}\right)\left(\mathscr{L} \otimes \mathbf{R} g_{*} \omega_{X}\right)\right)=\mathbf{h}^{i} \mathbf{R} \pi_{*}\left(g^{*} \mathscr{L} \otimes \omega_{X}\right) .
\end{aligned}
$$

This map is zero since the second line is zero by construction.

Corollary 5.6. Let $X$ be a projective variety over an $F$-finite field $k$. Suppose that $L$ is a Cartier divisor on $X$, and that $\Delta$ is a $\mathbb{Q}$-divisor such that $L-\left(K_{X}+\Delta\right)$ is a big and semi-ample $\mathbb{Q}$-Cartier $\mathbb{Q}$-divisor. Then there exists a finite surjective map $f: Y \rightarrow X$ from a normal variety $Y$ such that:

(a) The natural trace map

$$
f_{*} \mathscr{O}_{Y}\left(\left\lceil K_{Y}+f^{*}\left(L-\left(K_{X}+\Delta\right)\right\rceil\right) \rightarrow \mathscr{O}_{X}\left(\left\lceil K_{X}+L-\left(K_{X}+\Delta\right)\right\rceil\right)\right.
$$

has image $\tau(X ; \Delta) \otimes \mathscr{O}_{X}(L)$.

(b) The induced map on cohomology

$$
H^{i}\left(Y, \mathscr{O}_{Y}\left(\left\lceil K_{Y}+f^{*}\left(L-\left(K_{X}+\Delta\right)\right)\right\rceil\right)\right) \rightarrow H^{i}\left(X, \tau(X ; \Delta) \otimes \mathscr{O}_{X}(L)\right)
$$

is zero for all $i>0$.

\section{Transformation RUles For test ideals Under Alterations}

The fact that the test ideal can be computed via alterations suggests a transformation rule for test ideals under alterations. We derive this transformation rule in this section. We first state a definition.

Definition 6.1. Suppose that $X$ is a normal variety over a field $k$ and $\Gamma$ is a $\mathbb{Q}$-Cartier $\mathbb{Q}$-divisor. For example, one might take $\Gamma=L-\left(K_{X}+\Delta\right)$ where $L$ is a Cartier divisor and $K_{X}+\Delta$ is $\mathbb{Q}$-Cartier. We define

$$
T^{0}(X, \Gamma):=\bigcap_{f: Y \rightarrow X} \text { Image }\left(H^{0}\left(Y, \mathscr{O}_{Y}\left(\left\lceil K_{Y}+f^{*} \Gamma\right\rceil\right)\right) \stackrel{\operatorname{Tr}_{f}}{\longrightarrow} H^{0}\left(X, \mathscr{O}_{X}\left(\left\lceil K_{X}+\Gamma\right\rceil\right)\right)\right)
$$

where $f$ runs over all finite dominant maps $f: Y \rightarrow X$ such that $Y$ is normal and equidimensional.

Alternately, if $X$ is any (not necessarily normal or even reduced) $F$-finite $d$-dimensional equidimensional scheme of finite type over a field $k$ and $\mathscr{L}$ is any line bundle on $X$, then we define

$$
T^{0}(X, \mathscr{L}):=\bigcap_{f: Y \longrightarrow X} \text { Image }\left(H^{0}\left(Y, \omega_{Y} \otimes f^{*} \mathscr{L}\right) \stackrel{\operatorname{Tr}_{f}}{\longrightarrow} H^{0}\left(X, \omega_{X} \otimes \mathscr{L}\right)\right)
$$

where $f$ runs over all finite dominant maps $f: Y \rightarrow X$ such that $Y$ is normal and equidimensional of dimension $d$. In both cases the maps are induced by the trace map as described in Proposition 2.18.

Remark 6.2. We expect that the reader has noticed the upper-script 0 in the definition of $T^{0}(X, \Gamma)$. We include this for two reasons: 
(i) It serves to remind the reader that $T^{0}(X, \Gamma)$ is a submodule of $H^{0}\left(X, \mathscr{O}_{X}\left(\left\lceil K_{X}+\Gamma\right\rceil\right)\right)$.

(ii) In the future, it might be reasonable to extend this definition to higher cohomology groups. For example, Theorem 5.5 is a vanishing theorem for appropriately defined higher $T^{i}(X, \Gamma)$.

First we make a simple observation:

Lemma 6.3. For any finite dominant map $f: Y \rightarrow X$ between proper normal varieties over a field $k$ with $\Gamma$ a $\mathbb{Q}$-Cartier $\mathbb{Q}$-divisor on $X$, then $T^{0}\left(Y, f^{*} \Gamma\right)$ is sent onto $T^{0}(X, \Gamma)$ via the trace map

$$
\left.\beta: H^{0}\left(Y, \mathscr{O}_{Y}\left(\left\lceil K_{Y}+f^{*} \Gamma\right\rceil\right)\right) \rightarrow H^{0}\left(X, \mathscr{O}_{X}\left(\left\lceil K_{X}+\Gamma\right)\right\rceil\right)\right) .
$$

Proof. If $Y$ is proper over a field $k$, then $H^{0}\left(Y, \mathscr{O}_{Y}\left(\left\lceil K_{Y}+f^{*} \Gamma\right\rceil\right)\right)$ is a finite dimensional vector space, and so $T^{0}\left(Y, f^{*} \Gamma\right)$ is the image of a single map

$$
H^{0}\left(Y^{\prime}, \mathscr{O}_{Y^{\prime}}\left(\left\lceil K_{Y^{\prime}}+g^{*} f^{*} \Gamma\right\rceil\right)\right) \rightarrow H^{0}\left(Y, \mathscr{O}_{Y}\left(\left\lceil K_{Y}+f^{*} \Gamma\right\rceil\right)\right)
$$

for some finite cover $g: Y^{\prime} \rightarrow Y$. Composing with the map to $H^{0}\left(X, \mathscr{O}_{X}\left(\left\lceil K_{X}+\Gamma\right\rceil\right)\right)$ yields the inclusion $T^{0}(X, \Gamma) \subseteq \beta\left(T^{0}\left(Y, f^{*} \Gamma\right)\right)$.

For the other inclusion, we simply notice that given any finite dominant map $h: W \rightarrow X$, we can find a finite dominant map $U \rightarrow X$ which factors through both $h$ and $f$.

Lemma 6.4. Suppose that $X$ is as in Definition 6.1. Observe that $H^{0}\left(X, \omega_{X} \otimes \mathscr{L}\right) \cong$ $\mathbb{H}^{0}\left(X, \omega_{X}^{\cdot}[-\operatorname{dim} X] \otimes \mathscr{L}\right)$. Furthermore, $T^{0}\left(X, \omega_{X} \otimes \mathscr{L}\right)$ is:

$$
\bigcap_{f: Y \longrightarrow X} \operatorname{Image}\left(\mathbb{H}^{0}\left(Y, \omega_{Y}[-\operatorname{dim} X] \otimes f^{*} \mathscr{L}\right) \stackrel{\operatorname{Tr}_{f} \bullet}{\longrightarrow} \mathbb{H}^{0}\left(X, \omega_{X}[-\operatorname{dim} X] \otimes \mathscr{L}\right)\right) .
$$

where the intersection runs over all finite dominant maps $f: Y \rightarrow X$ such that $Y$ is normal and equidimensional.

Proof. First note that the isomorphism $H^{0}\left(X, \omega_{X} \otimes \mathscr{L}\right) \cong \mathbb{H}^{0}\left(X, \omega_{X}^{\cdot}[-\operatorname{dim} X] \otimes \mathscr{L}\right)$ follows from analyzing the spectral sequence computing $\mathbb{H}^{0}\left(X, \omega_{X}^{\cdot}[-\operatorname{dim} X] \otimes \mathscr{L}\right)$. The second statement then follows immediately.

Mimicking Lemma 6.3, we also obtain:

Lemma 6.5. For any finite dominant map $f: Y \rightarrow X$ between proper equidimensional schemes of finite type over a field $k$ with $\mathscr{L}$ a line bundle on $X$, then $T^{0}\left(Y, \omega_{Y} \otimes f^{*} \mathscr{L}\right)$ is sent onto $T^{0}\left(X, \omega_{X}\right)$ via the trace map

$$
\mathbb{H}^{0}\left(Y, \omega_{\dot{Y}}[-\operatorname{dim} X] \otimes f^{*} \mathscr{L}\right) \rightarrow \mathbb{H}^{0}\left(X, \omega_{X}^{\cdot}[-\operatorname{dim} X] \otimes \mathscr{L}\right)
$$

Proof. The proof is identical to the proof of Lemma 6.3.

By Theorem 4.6, if $X=\operatorname{Spec} R$ is affine and $L=0$, then $T^{0}\left(X, L-\left(K_{X}+\Delta\right)\right)$ is just the global sections of $\tau(X ; \Delta)$. Inspired by this, we demonstrate that we may also use alterations in order to compute $T^{0}\left(X, L-\left(K_{X}+\Delta\right)\right)$.

First, suppose that $f: Y \rightarrow X$ is an alteration and recall from Proposition 2.18 that we have a map

$$
\operatorname{Tr}_{f}: f_{*} \omega_{Y}\left(-\left\lfloor f^{*}\left(K_{X}+\Delta\right)\right\rfloor\right) \rightarrow \omega_{X}\left(-\left\lfloor K_{X}+\Delta\right\rfloor\right) \text {. }
$$

Twisting by a Cartier divisor $L$ and taking cohomology leads us to a map

$$
\begin{aligned}
\Psi: H^{0}\left(Y, \omega_{Y}\left(\left\lceil f^{*}\left(L-K_{X}-\Delta\right)\right\rceil\right)\right) & =H^{0}\left(X, f_{*} \omega_{Y}\left(\left\lceil f^{*}\left(L-K_{X}-\Delta\right)\right\rceil\right)\right) \\
& \rightarrow H^{0}\left(X, \omega_{X}\left(\left\lceil L-K_{X}-\Delta\right\rceil\right)\right) .
\end{aligned}
$$


Theorem 6.7. Suppose that $X$ is a $F$-finite normal variety over $k$ and that $\Delta$ is a $\mathbb{Q}$-divisor such that $K_{X}+\Delta$ is $\mathbb{Q}$-Cartier. Finally set $L$ to be any Cartier divisor. Then

$$
\begin{aligned}
& T^{0}\left(X, L-K_{X}-\Delta\right) \\
& \quad=\bigcap_{f: Y \rightarrow X} \text { Image }\left(H^{0}\left(Y, \omega_{Y}\left(\left\lceil f^{*}\left(L-K_{X}-\Delta\right)\right\rceil\right)\right) \stackrel{\operatorname{Tr}_{f}}{\longrightarrow} H^{0}\left(X, \omega_{X}\left(\left\lceil L-K_{X}-\Delta\right\rceil\right)\right)\right)
\end{aligned}
$$

where $f$ runs over all alterations $f: Y \rightarrow X$ and the maps in the intersection are as in (6.6).

Proof. Certainly we have the containment $T^{0}\left(X, L-\left(K_{X}+\Delta\right)\right) \supseteq \bigcap_{f: Y \rightarrow X}(\ldots)$ since this latter intersection intersects more modules than the one defining $T^{0}\left(X, L-\left(K_{X}+\Delta\right)\right)$. We need to prove the reverse containment.

Fix an alteration $f: Y \rightarrow X$. Set $Y \stackrel{\alpha}{\longrightarrow} W=\operatorname{Spec}\left(f_{*} \mathscr{O}_{Y}\right) \stackrel{\beta}{\longrightarrow} X$ to be the Stein factorization of $f$. Certainly

$$
\tau\left(\omega_{W}, \beta^{*}\left(L-K_{X}-\Delta\right)\right) \subseteq \alpha_{*} \mathscr{O}_{Y}\left(\left\lceil K_{Y}+f^{*}\left(L-K_{X}-\Delta\right)\right\rceil\right) .
$$

Choose a finite cover $h: U \rightarrow W$ such that $\operatorname{Tr}_{h}: \mathscr{O}_{U}\left(\left\lceil K_{U}-h^{*} \beta^{*}\left(L-K_{X}-\Delta\right)\right\rceil\right) \rightarrow$ $\mathscr{O}_{W}\left(\left\lceil K_{W}-\beta^{*}\left(L-K_{X}-\Delta\right)\right\rceil\right)$ has image $\tau\left(\omega_{W}, \beta^{*}\left(L-K_{X}-\Delta\right)\right)$.

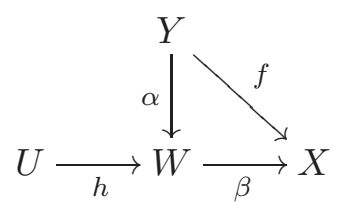

We now have the following factorization:

$$
\begin{aligned}
H^{0}\left(U, \mathscr{O}_{U}\left(\left\lceilK_{U}+h^{*}\right.\right.\right. & \left.\left.\left.\beta^{*}\left(L-K_{X}-\Delta\right)\right\rceil\right)\right) \rightarrow H^{0}\left(X, \beta_{*} \tau\left(\omega_{W}, \beta^{*}\left(L-K_{X}-\Delta\right)\right)\right) \\
& \rightarrow H^{0}\left(X, \beta_{*} \alpha_{*} \mathscr{O}_{Y}\left(\left\lceil K_{Y}+f^{*}\left(L-K_{X}-\Delta\right)\right\rceil\right)\right) \\
& =H^{0}\left(X, f_{*} \mathscr{O}_{Y}\left(\left\lceil K_{Y}+f^{*}\left(L-K_{X}-\Delta\right)\right\rceil\right)\right) \\
& =H^{0}\left(Y, \omega_{Y}\left(\left\lceil f^{*}\left(L-K_{X}-\Delta\right)\right)\right\rceil\right) \\
& \rightarrow H^{0}\left(X, \omega_{X}\left(\left\lceil L-K_{X}-\Delta\right\rceil\right)\right)
\end{aligned}
$$

And so we obtain the desired inclusion.

Theorem 6.8. Suppose that $f: Z \rightarrow X$ is an alteration between normal $F$-finite varieties over a field $k, \Delta$ is a $\mathbb{Q}$-divisor on $X$ such that $K_{X}+\Delta$ is $\mathbb{Q}$-Cartier and $L$ is any Cartier divisor on $X$. Additionally suppose that either $X$ is affine or proper over $k$.

Then

$$
\Psi\left(T^{0}\left(Z, f^{*}\left(L-\left(K_{X}+\Delta\right)\right)\right)\right)=T^{0}\left(X, L-\left(K_{X}+\Delta\right)\right)
$$

where $\Psi$ is the map described in (6.6).

In particular, if $f$ is proper and birational and $X$ is affine, then this yields a transformation rule for the test ideal under proper birational morphisms.

Proof. Certainly $T^{0}\left(X, L-\left(K_{X}+\Delta\right)\right) \supseteq \Psi\left(T^{0}\left(Z, f^{*}\left(L-\left(K_{X}+\Delta\right)\right)\right)\right)$ in either case by Theorem 6.7.

If $X$ is proper over $k$, then so is $Z$ and so $H^{0}\left(Z, \mathscr{O}_{Z}\left(\left\lceil K_{Z}+f^{*}\left(L-\left(K_{X}+\Delta\right)\right)\right\rceil\right)\right)$ is a finite dimensional $k$-vector space. It follows that $T^{0}\left(Z, f^{*}\left(L-\left(K_{X}+\Delta\right)\right)\right)$ is the image of a single map

$$
H^{0}\left(Z^{\prime}, \mathscr{O}_{Z^{\prime}}\left(\left\lceil K_{Z^{\prime}}+g^{*} f^{*}\left(L-K_{X}-\Delta\right)\right\rceil\right)\right) \stackrel{\operatorname{Tr}_{g}}{\longrightarrow} H^{0}\left(Z, \mathscr{O}_{Z}\left(\left\lceil K_{Z}+f^{*}\left(L-K_{X}-\Delta\right)\right\rceil\right)\right)
$$


for some finite cover $g: Z^{\prime} \rightarrow Z$. However, $f \circ g: Z^{\prime} \rightarrow X$ is also an alteration, and it follows that $T^{0}\left(X, L-\left(K_{X}+\Delta\right)\right)$ is contained in the image of

$$
H^{0}\left(Z^{\prime}, \mathscr{O}_{Z^{\prime}}\left(\left\lceil K_{Z^{\prime}}+g^{*} f^{*}\left(L-K_{X}-\Delta\right)\right\rceil\right)\right) \stackrel{\operatorname{Tr}_{f \circ g}}{\longrightarrow} H^{0}\left(X, \mathscr{O}_{X}\left(\left\lceil K_{X}+L-K_{X}-\Delta\right\rceil\right)\right)
$$

whose image is clearly $\operatorname{Tr}_{f}\left(T^{0}\left(Z, f^{*}\left(L-\left(K_{X}+\Delta\right)\right)\right)\right)$.

On the other hand, suppose $X=\operatorname{Spec} R$ is affine and observe that, without loss of generality, we may assume that $K_{X}+\Delta$ is effective. Set $S=H^{0}\left(Y, \mathscr{O}_{Y}\right)$ and define $Y \stackrel{\alpha}{\longrightarrow} X^{\prime}=\operatorname{Spec} S \stackrel{\beta}{\longrightarrow} X$ to be the Stein factorization of $f$. Now, the global sections $H^{0}\left(Z, \mathscr{O}_{Z}\left(\left\lceil K_{Z}+f^{*}\left(L-\left(K_{X}+\Delta\right)\right)\right\rceil\right)\right)$ can be identified with elements of $\omega_{S}(L)$ because we assumed that $K_{X}+\Delta \geq 0$. In particular, we have $\tau\left(\omega_{S}, \beta^{*}\left(L-K_{X}-\Delta\right)\right) \subseteq$ $H^{0}\left(Z, \mathscr{O}_{Z}\left(\left\lceil K_{Z}-f^{*}\left(L-\left(K_{X}+\Delta\right)\right)\right\rceil\right)\right)$. But $\operatorname{Tr}_{\beta}\left(\tau\left(\omega_{S}, \beta^{*}\left(L-K_{X}-\Delta\right)\right)\right)=\tau\left(R, L-K_{X}-\Delta\right)$ and the other containment follows.

Remark 6.9. In order to generalize the above result to arbitrary schemes, it would be helpful to know that the intersection defining $T^{0}(X, \Gamma)$ stabilized in general.

\section{Surjectivities on COHOMOlOGy}

In this section we show how the vanishing statements obtained in Bha10, and in Section 5 , combined with the ideas of Section 6, can be used to construct global sections of adjoint line bundles. We are treating this current section as a proof-of-concept. In particular, many of the statements can be easily generalized. We leave the statements simple however in order to demonstrate the main ideas. Consider the following prototypical application of Kodaira vanishing.

Example 7.1. Suppose that $X$ is a smooth projective variety in characteristic zero and that $D$ is an effective Cartier divisor on $X$. Set $\mathscr{L}$ to be an ample line bundle on $X$. We have the following short exact sequence

$$
\left.0 \rightarrow \omega_{X} \otimes \mathscr{L} \rightarrow \omega_{X}(D) \otimes \mathscr{L} \rightarrow \omega_{D} \otimes \mathscr{L}\right|_{D} \rightarrow 0
$$

Taking cohomology gives us

$$
0 \rightarrow H^{0}\left(X, \omega_{X} \otimes \mathscr{L}\right) \rightarrow H^{0}\left(X, \omega_{X}(D) \otimes \mathscr{L}\right) \rightarrow H^{0}\left(D,\left.\omega_{D} \otimes \mathscr{L}\right|_{D}\right) \rightarrow H^{1}\left(X, \omega_{X} \otimes \mathscr{L}\right) .
$$

Kodaira vanishing implies that $H^{1}\left(X, \omega_{X} \otimes \mathscr{L}\right)$ is zero and so

$$
H^{0}\left(X, \omega_{X}(D) \otimes \mathscr{L}\right) \rightarrow H^{0}\left(D,\left.\omega_{D} \otimes \mathscr{L}\right|_{D}\right)
$$

is surjective.

Consider now the same example (in characteristic zero) but do not assume that $X$ is smooth.

Example 7.2. Suppose that $X$ is a normal projective variety in characteristic zero and that $D$ is a reduced Cohen-Macaulay Cartier divisor on $X$. These conditions are enough to imply that the natural map $\omega_{X}(D) \rightarrow \omega_{D}$ is surjective.

Set $\mathscr{L}$ to be an ample (or even big and nef) line bundle on $X$. Choose $\pi: \widetilde{X} \rightarrow X$ to be a $\log$ resolution of $(X, D)$ and set $\widetilde{D}$ to be the strict transform of $D$ on $\widetilde{X}$. We have the 
following diagram of exact triangles in $D_{\text {coh }}^{b}(X)$ :

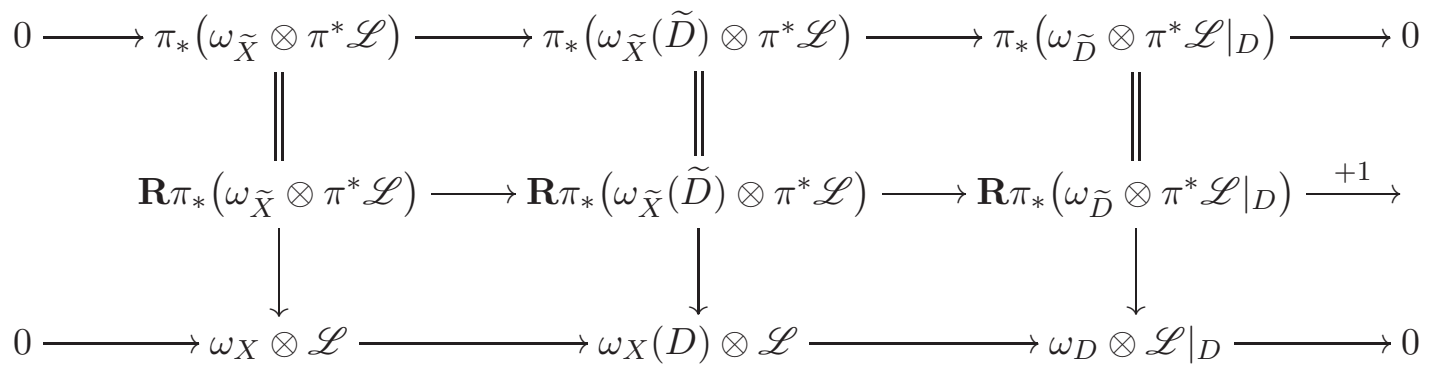

The vertical equalities and the top right surjection are due Grauert-Riemenschneider vanishing [GR70]. Because $X$ is not smooth, $H^{1}\left(X, \omega_{X}(L)\right)$ is not necessarily zero, see for example AJ89. However, $H^{1}\left(X,\left(\pi_{*} \omega_{\widetilde{X}}\right) \otimes \mathscr{L}\right)=H^{1}\left(\widetilde{X}, \omega_{\widetilde{X}} \otimes \pi^{*} \mathscr{L}\right)$ is zero by Kawamata-Viehweg vanishing since $\pi^{*} L$ is big and nef, Kaw82, Vie82]. Thus we have the surjection:

$$
H^{0}\left(X,\left(\pi_{*} \omega_{\widetilde{X}}(\widetilde{D})\right) \otimes \mathscr{L}\right) \rightarrow H^{0}\left(X,\left.\left(\pi_{*} \omega_{\widetilde{D}}\right) \otimes \mathscr{L}\right|_{D}\right),
$$

between submodules of $H^{0}\left(X, \omega_{X}(D) \otimes \mathscr{L}\right)$ and $H^{0}\left(D,\left.\omega_{D} \otimes \mathscr{L}\right|_{D}\right)$.

Interestingly, $\pi_{*} \omega_{\widetilde{D}}$ is independent of the choice of embedding of $D$ into $X$ since $\pi_{*} \omega_{\widetilde{D}}$ is the multiplier module for $D$ by definition. Even more, $H^{0}\left(D,\left.\pi_{*} \omega_{\widetilde{D}} \otimes \mathscr{L}\right|_{D}\right)$ only depends on the pair $\left(D,\left.\mathscr{L}\right|_{D}\right)$.

Furthermore, using the method of proof of Theorem 8.3 below, it is easy to see that

$$
H^{0}\left(D,\left.\pi_{*} \omega_{\widetilde{D}} \otimes \mathscr{L}\right|_{D}\right)=\bigcap_{f: E \longrightarrow D} \operatorname{Image}\left(H^{0}\left(E,\left.\omega_{E} \otimes f^{*} \mathscr{L}\right|_{D}\right) \rightarrow H^{0}\left(D,\left.\omega_{D} \otimes \mathscr{L}\right|_{D}\right)\right)
$$

where the intersection runs over all regular alterations $f: E \rightarrow D$. In light of Theorem 6.7 the subspace $H^{0}\left(D,\left.\pi_{*} \omega_{\widetilde{D}} \otimes \mathscr{L}\right|_{D}\right)$ may be viewed as an analog of $T^{0}\left(D,\left.\omega_{\widetilde{D}} \otimes \mathscr{L}\right|_{D}\right)$. This inspires the remainder of the section.

In characteristic $p>0$, Kodaira vanishing does not hold even on smooth varieties Ray78. However, we have the following corollary of [Bha10], $c f$. Corollary 5.6.

Theorem 7.3. Suppose that $D$ is a Cartier divisor on a normal proper d-dimensional variety $X$ and $\mathscr{L}$ is a big and semi-ample line bundle on $X$. Using the natural map

$$
\gamma: H^{0}\left(X, \omega_{X}(D) \otimes \mathscr{L}\right) \rightarrow H^{0}\left(D,\left.\omega_{D} \otimes \mathscr{L}\right|_{D}\right)
$$

one has an inclusion

$$
T^{0}\left(D,\left.\omega_{D} \otimes \mathscr{L}\right|_{D}\right) \subseteq \gamma\left(T^{0}\left(X, \omega_{X} \otimes \mathscr{L}(D)\right)\right) .
$$

In particular, if $T^{0}\left(D,\left.\omega_{D} \otimes \mathscr{L}\right|_{D}\right) \neq 0$, then $H^{0}\left(X, \omega_{X}(D) \otimes \mathscr{L}\right) \neq 0$. And if $T^{0}\left(D, \omega_{D} \otimes\right.$ $\left.\left.\mathscr{L}\right|_{D}\right)=H^{0}\left(D,\left.\omega_{D} \otimes \mathscr{L}\right|_{D}\right)$ then $\gamma$ is surjective.

Proof. Using Lemma 6.4, set $f: Y \rightarrow X$ to be a finite cover of $X$ such that $T^{0}\left(X, \omega_{X} \otimes\right.$ $\mathscr{L}(D))$ is equal to

$$
\text { Image }\left(\mathbb{H}^{0}\left(Y, \omega_{\dot{Y}}[-d] \otimes f^{*} \mathscr{L}(D)\right) \stackrel{\operatorname{Tr}_{f} \bullet}{\longrightarrow} \mathbb{H}^{0}\left(X, \omega_{X}[-d] \otimes \mathscr{L}(D)\right)\right)
$$

noting that $\mathbb{H}^{0}\left(X, \omega_{X}^{\cdot}[-d] \otimes \mathscr{L}(D)\right) \cong H^{0}\left(X, \omega_{X} \otimes \mathscr{L}(D)\right)$. By Bha10, Proposition 5.5.3], there exists a finite cover $g: Z \rightarrow Y$ such that $H^{d-1}\left(Y, f^{*} \mathscr{L}^{-1}\right) \rightarrow H^{d-1}\left(Z, g^{*} f^{*} \mathscr{L}^{-1}\right)$ is the zero map. Therefore the dual map $\mathbb{H}^{1-d}\left(Z, g^{*} f^{*} \mathscr{L} \otimes \omega_{Y}^{\bullet}\right) \rightarrow \mathbb{H}^{1-d}\left(Y, f^{*} \mathscr{L} \otimes \omega_{X}^{*}\right)$ is zero. 
Set $D_{Y}=f^{*} D$ and $D_{Z}=g^{*} f^{*} D$. Note that $D_{Y}$ or $D_{Z}$ may not be normal or even reduced, even if $D$ is. They are however equidimensional. Then there is a map between long exact sequences:

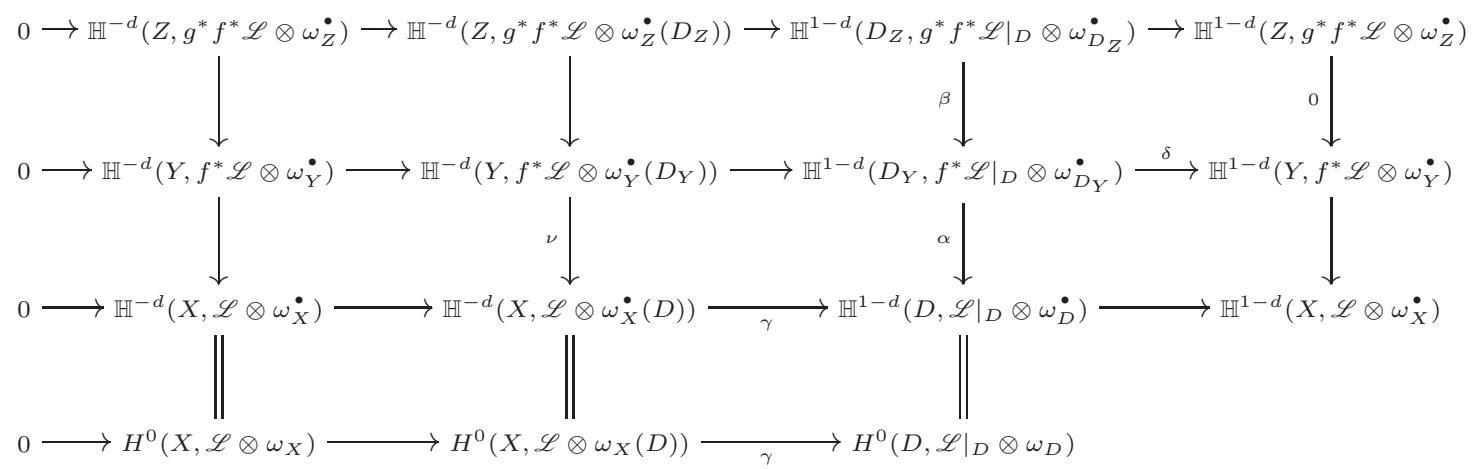

where the vertical equalities are obtained from the spectral sequences computing the middle lines. Note we identify $f$ with its restriction $\left.f\right|_{D_{Y}}$ and $g$ with $\left.g\right|_{D_{Z}}$

Choose the $h: E \rightarrow D_{Z}$ to be normalization of the $\left(D_{Z}\right)_{\text {red }}$ and notice we have a map

$$
\begin{aligned}
H^{0}\left(E,\left.\omega_{E} \otimes h^{*} g^{*} f^{*} \mathscr{L}\right|_{D}\right) & =\mathbb{H}^{1-d}\left(E,\left.\omega_{E}^{\bullet} \otimes h^{*} g^{*} f^{*} \mathscr{L}\right|_{D}\right) \\
& \longrightarrow \mathbb{H}^{1-d}\left(D_{Z},\left.\omega_{D_{Z}} \otimes g^{*} f^{*} \mathscr{L}\right|_{D}\right) \\
& \stackrel{\alpha \circ \beta}{\longrightarrow} \mathbb{H}^{1-d}\left(D,\left.\omega_{D} \otimes \mathscr{L}\right|_{D}\right)=H^{0}\left(D,\left.\omega_{D} \otimes \mathscr{L}\right|_{D}\right) .
\end{aligned}
$$

The image of this map contains $T^{0}\left(D,\left.\omega_{D} \otimes \mathscr{L}\right|_{D}\right)$, and thus the image of $\alpha \circ \beta$ also contains $T^{0}\left(D,\left.\omega_{D} \otimes \mathscr{L}\right|_{D}\right)$. Therefore, if we view $d \in T^{0}\left(D, \omega_{D} \otimes \mathscr{M}\right)$ as an element of $\mathbb{H}^{1-d}\left(D,\left.\mathscr{L}\right|_{D} \otimes\right.$ $\left.\omega_{D}^{\bullet}\right)$, it must have some pre-image $z \in \mathbb{H}^{1-d}\left(D_{Z},\left.f^{*} \mathscr{L}\right|_{D} \otimes \omega_{D_{Z}}\right)$. The diagram implies that $\delta(\beta(z))=0$. Thus $\beta(z)$ is an image of some element in $y \in H^{0}\left(X, f^{*} \mathscr{L} \otimes \omega_{Y}^{\bullet}\left(D_{Y}\right)\right)$. It follows that $\nu(y) \in T^{0}\left(X, \omega_{X} \otimes \mathscr{L}(D)\right)$ and so $d=\gamma(\nu(y))$ which completes the proof.

Remark 7.4. If we knew that the intersection defining $T^{0}\left(X, L-K_{X}-\Delta\right)$ stabilized, then the previous result could be generalized to arbitrary equidimensional schemes (not just those which are of finite type over a field). Even without this hypothesis, the argument above still implies that $T^{0}\left(D,\left.\omega_{D} \otimes \mathscr{L}\right|_{D}\right) \subseteq \gamma\left(H^{0}\left(X, \omega_{X}(D) \otimes \mathscr{L}\right)\right)$. The same statement holds for Theorem 7.6.

Remark 7.5. We expect that one can obtain more precise surjectivities involving characteristic $p>0$ analogs of adjoint ideals. In particular, $T^{0}\left(X, \omega_{X} \otimes \mathscr{L}\right)$ is not the right analog of the term $H^{0}\left(X, \pi_{*} \omega_{\widetilde{X}}(\widetilde{D})\right)$ appearing in Example 7.2 above.

We also show that this method can be generalized with $\mathbb{Q}$-divisors.

Theorem 7.6. Suppose $X$ is a normal $F$-finite variety which is proper over a field $k$ and that $D$ is a Cartier divisor on $X$. Additionally, suppose that $\Delta$ is a $\mathbb{Q}$-divisor on $X$ with no common components with $D$ and such that $K_{X}+\Delta$ is $\mathbb{Q}$-Cartier. Finally suppose that $L$ is a Cartier divisor on $X$ such that $L-\left(K_{X}+D+\Delta\right)$ is big and semi-ample. Then the natural map

$$
\begin{aligned}
H^{0}\left(X, \mathscr{O}_{X}\left(\left\lceil K_{X}+D+L-\left(K_{X}+D+\Delta\right)\right\rceil\right)=H^{0}\left(X, \mathscr{O}_{X}(\lceil L-\Delta\rceil)\right.\right. \\
\quad \stackrel{\gamma}{\longrightarrow} H^{0}\left(D, \mathscr{O}_{D}\left(\left\lceil K_{D}+\left.L\right|_{D}-\left(K_{D}+\left.\Delta\right|_{D}\right)\right\rceil\right)\right)=H^{0}\left(D, \mathscr{O}_{D}\left(\left.L\right|_{D}-\left.\lfloor\Delta\rfloor\right|_{D}\right) .\right.
\end{aligned}
$$

yields an inclusion

$$
T^{0}\left(D,\left.L\right|_{D}-\left(K_{D}+\left.\Delta\right|_{D}\right)\right) \subseteq \gamma\left(T^{0}\left(X, D+L-\left(K_{X}+D+\Delta\right)\right)\right)
$$


noting that $T^{0}\left(D,\left.L\right|_{D}-\left(K_{D}+\left.\Delta\right|_{D}\right)\right) \subseteq H^{0}\left(D, \mathscr{O}_{D}\left(\left\lceil\left. L\right|_{D}-\left.\lfloor\Delta\rfloor\right|_{D}\right\rceil\right)\right)$.

Proof. Let us first point out that $\gamma$ is induced from the restriction map

$$
\begin{aligned}
\mathscr{O}_{X}\left(\left\lceil K_{X}+D+L-\left(K_{X}+D+\Delta\right)\right\rceil\right) & \\
& \rightarrow \mathscr{O}_{D}\left(\left.\left\lceil K_{X}+D+L-\left(K_{X}+D+\Delta\right)\right\rceil\right|_{D}\right)=\mathscr{O}_{D}\left(K_{D}+\left.L\right|_{D}-\left(K_{D}+\left.\lfloor\Delta\rfloor\right|_{D}\right)\right) .
\end{aligned}
$$

Now choose a finite cover $h: W \rightarrow X$, with $W$ normal, such that $h^{*}\left(K_{X}+\Delta\right)$ is an integral Cartier divisor and set $D_{W}=h^{*} D$. Note $D_{W}$ is not necessarily normal or even reduced (it is however unmixed and thus equidimensional). We have the diagram

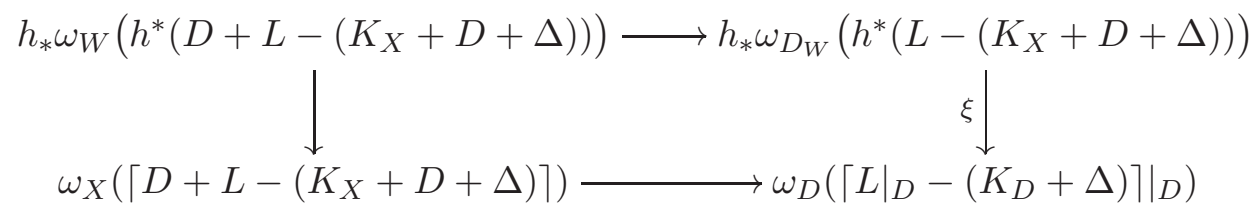

of which we take global sections and then apply the method of Lemmas 6.3 and 6.5 to conclude that the image $\xi\left(T^{0}\left(D_{W}, \omega_{D_{W}} \otimes \mathscr{O}_{W}\left(h^{*}\left(L-\left(K_{X}+D+\Delta\right)\right)\right)\right)\right)$ is equal to

$$
\begin{aligned}
T^{0}\left(D,\left.L\right|_{D}-\left(K_{D}+\left.\Delta\right|_{D}\right)\right) & \subseteq H^{0}\left(D, \mathscr{O}_{D}\left(K_{D}+\left.L\right|_{D}-\left(K_{D}+\left.\Delta\right|_{D}\right)\right)\right) \\
& \subseteq H^{0}\left(D, \omega_{D}\left(\left.\left\lceil\left. L\right|_{D}-\left(K_{D}+\Delta\right)\right\rceil\right|_{D}\right)\right) .
\end{aligned}
$$

Likewise $T^{0}\left(X, D+L-\left(K_{X}+D+\Delta\right)\right)$ is the image of $T^{0}\left(W, h^{*}\left(D+L-\left(K_{X}+D+\Delta\right)\right)\right)$. Thus it is sufficient to show that via the map

$$
H^{0}\left(W, \omega_{W}\left(h^{*}\left(D+L-\left(K_{X}+D+\Delta\right)\right)\right)\right) \rightarrow H^{0}\left(D_{W}, \omega_{D_{W}}\left(h^{*}\left(L-\left(K_{X}+D+\Delta\right)\right)\right)\right),
$$

each element of $T^{0}\left(D_{W}, \omega_{D_{W}} \otimes \mathscr{O}_{W}\left(h^{*}\left(L-\left(K_{X}+D+\Delta\right)\right)\right)\right)$ is the image of an element of $T^{0}\left(W, \omega_{W} \otimes \mathscr{O}_{W}\left(h^{*}\left(D+L-\left(K_{X}+D+\Delta\right)\right)\right)\right)$. We have just reduced to the setting of Theorem 7.3 and the result follows.

\section{TRANSFORMATION RULES FOR MULTIPLIER IDEALS}

It still remains to be proven that the multiplier ideal in characteristic zero can be characterized as in our Main Theorem, for which we need to explore the behavior of multiplier ideals under proper dominant maps. We further analyze the behavior of multiplier ideals under alterations in arbitrary characteristic, which leads to an understanding of when (and why) the classical characteristic zero transformation rule (2.41) for the multiplier ideal under finite maps may fail in positive characteristic.

Suppose that $A$ is a normal ring in arbitrary characteristic and that $(X=\operatorname{Spec} A, \Delta)$ is an affine pair such that $K_{X}+\Delta$ is $\mathbb{Q}$-Cartier. As discussed in Section 2.4, $\mathcal{J}(X ; \Delta)$ is only known to be quasi-coherent assuming a theory of resolution of singularities is at hand. Nonetheless, it is always a sheaf of (fractional) ideals, and in this section we will use the notation $\mathcal{J}(A ; \Delta):=H^{0}(X, \mathcal{J}(X ; \Delta))$ to denote the corresponding ideal of global sections.

An important and useful perspective, largely in the spirit of Lip94, is to view Definition 2.28 as a collection of valuative conditions for membership in the multiplier ideal $\mathcal{J}(A ; \Delta)$. Specifically, suppose $E$ is a prime divisor on a normal proper birational modification $\theta: Z \rightarrow X$. After identification of the function fields $K=\operatorname{Frac}(A)=K(X)=K(Z)$, $E$ gives rise to a valuation $\operatorname{ord}_{E}$ centered on $X$. The valuation ring of $\operatorname{ord}_{E}$ is simply the local ring $\mathscr{O}_{Z, E}$. Thus, we have that $\mathcal{J}(A ; \Delta)$ can be described as the fractional ideal inside of $K(X)$ given by 


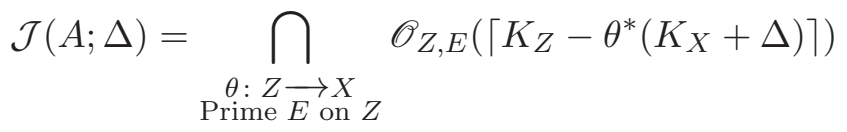

$$
\begin{aligned}
& =\left\{\begin{array}{l|l}
f \in K & \begin{array}{c}
\operatorname{ord}_{E}(f) \geq \operatorname{ord}_{E}\left(\left\lfloor\theta^{*}\left(K_{X}+\Delta\right)-K_{Z}\right\rfloor\right) \\
\text { for all } \theta: Z \rightarrow X \text { and all prime } E \text { on } Z
\end{array}
\end{array}\right\}
\end{aligned}
$$

We now show how to generalize the characteristic zero transformation rule for multiplier ideals under finite maps (2.41) so as to incorporate the trace map as in (2.42). Furthermore, by working in arbitrary characteristic, we also recover both of these transformation rules in positive characteristic for separable finite maps of degree prime to the characteristic.

Theorem 8.1. Suppose that $\pi: \operatorname{Spec} B=Y \rightarrow X=\operatorname{Spec} A$ is a finite dominant map of normal integral schemes of any characteristic and that $\left(X, \Delta_{X}\right)$ is a pair such that $K_{X}+\Delta_{X}$ is $\mathbb{Q}$-Cartier. Define $\Delta_{Y}=\Delta_{X}-\operatorname{Ram}_{\pi}$. Then

$$
\operatorname{Tr}\left(\mathcal{J}\left(B ; \Delta_{Y}\right)\right) \subseteq \mathcal{J}\left(A ; \Delta_{X}\right) .
$$

Furthermore, if the field trace map $\operatorname{Tr}: \operatorname{Frac}(B) \rightarrow \operatorname{Frac}(A)$ satisfies $\operatorname{Tr}(B)=A$ (e.g. the degree of $\pi$ is prime to the characteristic), then

$$
\operatorname{Tr}\left(\mathcal{J}\left(B ; \Delta_{Y}\right)\right)=\mathcal{J}\left(B ; \Delta_{Y}\right) \cap K(A)=\mathcal{J}\left(A ; \Delta_{X}\right) .
$$

Proof. Suppose $f \in \mathcal{J}\left(B ; \Delta_{Y}\right)$. Fix a prime divisor $E$ on a normal proper birational modification $\theta: Z \rightarrow X$. Consider the discrete valuation $\operatorname{ring} R=\mathscr{O}_{Z, E}$, viewed as a subring of $K(A)$, and let $r \in K(A)$ be a uniformizer for $R$. Denote by $S$ the integral closure of $R$ inside of $K(B)$. Then $S$ can also be realized in the following manner. Let $W$ be the normal scheme fitting into a commutative diagram

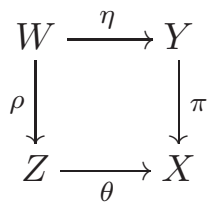

where $\rho$ is finite and $\eta$ is birational (that is, take $W$ to be the normalization of the relevant irreducible component of $Y \times_{X} Z$ ). Let $E_{1}, \ldots, E_{k}$ be the prime divisors on $W$ mapping onto $E$. Then we have $S=\bigcap_{i=1}^{k} \mathscr{O}_{W, E_{i}}$, where again we have considered each $\mathscr{O}_{W, E_{i}}$ as a subring of $K(B)$. In particular, for $g \in K(B)$, we have $g \in S$ if and only if $\operatorname{ord}_{E_{i}}(g) \geq 0$ for all $i=1, \ldots, k$.

Let $\Phi$ be a generator for the rank one free $S$-module $\operatorname{Hom}_{R}(S, R)$. If we write $\operatorname{Tr}: S \rightarrow R$ as $\operatorname{Tr}\left(\_\right)=\Phi\left(c \cdot{ }_{-}\right)$, we know from [ST10, Proposition 4.8] that $\operatorname{div}_{W}(c)=\operatorname{Ram}_{\rho}$ so that

$$
\operatorname{ord}_{E_{i}}(c)=\operatorname{ord}_{E_{i}}\left(K_{W}-\rho^{*} K_{Z}\right) \text {. }
$$

Let $\lambda_{E}=\operatorname{ord}_{E}\left(\left\lceil K_{Z}-\theta^{*}\left(K_{X}+\Delta_{X}\right)\right\rceil\right)$ and consider $g=c r^{\lambda_{E}} f$. Since $f \in \mathcal{J}\left(B ; \Delta_{Y}\right)$, it follows that $\operatorname{ord}_{E_{i}}(f)+\left\lceil K_{W}-\eta^{*}\left(K_{Y}+\Delta_{Y}\right)\right\rceil \geq 0$ and hence

$$
\begin{aligned}
\operatorname{ord}_{E_{i}}(g) & \geq \operatorname{ord}_{E_{i}}\left(K_{W}-\rho^{*} K_{Z}+\rho^{*}\left\lceil K_{Z}-\theta^{*}\left(K_{X}+\Delta_{X}\right)\right\rceil-\left\lceil K_{W}-\eta^{*}\left(K_{Y}+\Delta_{Y}\right)\right\rceil\right) \\
& =\operatorname{ord}_{E_{i}}\left(\rho^{*}\left\lceil-\theta^{*}\left(K_{X}+\Delta_{X}\right)\right\rceil-\left\lceil-\eta^{*}\left(K_{Y}+\Delta_{Y}\right)\right\rceil\right) \\
& =\operatorname{ord}_{E_{i}}\left(\rho^{*}\left\lceil-\theta^{*}\left(K_{X}+\Delta_{X}\right)\right\rceil-\left\lceil-\eta^{*}\left(K_{Y}+\pi^{*} \Delta_{X}-\left(K_{Y}-\pi^{*} K_{X}\right)\right)\right\rceil\right) \\
& =\operatorname{ord}_{E_{i}}\left(\rho^{*}\left\lceil-\theta^{*}\left(K_{X}+\Delta_{X}\right)\right\rceil-\left\lceil\rho^{*}\left(-\theta^{*}\left(K_{X}+\Delta_{X}\right)\right)\right\rceil\right) \\
& \geq 0 .
\end{aligned}
$$


It now follows that $g \in S$, and thus $\Phi(g)=r^{\lambda_{E}} \operatorname{Tr}(f) \in R$. In other words, we have

$$
\begin{aligned}
\operatorname{ord}_{E}(\Phi(g)) & =\operatorname{ord}_{E}(\operatorname{Tr}(f))+\operatorname{ord}_{E}\left(r^{\lambda_{E}}\right) \\
& =\operatorname{ord}_{E}(\operatorname{Tr}(f))+\operatorname{ord}_{E}\left(\left\lceil K_{Z}-\theta^{*}\left(K_{X}+\Delta_{X}\right\rceil\right) \geq 0\right.
\end{aligned}
$$

and we conclude that $\operatorname{Tr}(f) \in \mathcal{J}\left(A ; \Delta_{X}\right)$ and thus $\operatorname{Tr}\left(\mathcal{J}\left(B ; \Delta_{Y}\right)\right) \subseteq \mathcal{J}\left(B ; \Delta_{X}\right)$ as desired.

Note that every divisorial valuation $\nu: K(B) \backslash\{0\} \rightarrow \mathbb{Z}$ centered on $Y$ can be realized as $\nu=\operatorname{ord}_{E_{i}}$ as in the setup above. Indeed, the restriction $\nu$ to $K(A)$ gives rise to a discrete valuation ring whose residue field has transcendence degree $(\operatorname{dim}(Y)-1)=(\operatorname{dim}(X)-1)$ over $\Lambda$; see [Bou98, Chapter VI, Section 8]. By Proposition 2.45 in [KM98, this valuation ring can be realized as $\mathscr{O}_{Z, E}$ for some prime divisor $E$ on $\theta: Z \rightarrow X$ as above, so that $\nu=\operatorname{ord}_{E_{i}}$ for some $i$.

Let us now argue that $\mathcal{J}\left(A ; \Delta_{X}\right) \cdot \pi_{*} \mathscr{O}_{Y} \subseteq \pi_{*} \mathcal{J}\left(B ; \Delta_{Y}\right)$. Suppose $h \in \mathcal{J}\left(A ; \Delta_{X}\right)$. We have by assumption

$$
0 \leq \operatorname{ord}_{E}(h)+\operatorname{ord}_{E}\left(\left\lceil K_{Z}-\theta^{*}\left(K_{X}+\Delta_{X}\right)\right\rceil\right)
$$

whence it follows from [Har77, Chapter IV, Proposition 2.2], that

$$
\begin{aligned}
0 & \leq \operatorname{ord}_{E_{i}}(h)+\operatorname{ord}_{E_{i}}\left(\rho^{*}\left\lceil K_{Z}-\theta^{*}\left(K_{X}+\Delta_{X}\right)\right\rceil\right) \\
& \leq \operatorname{ord}_{E_{i}}(h)+\operatorname{ord}_{E_{i}}\left(\operatorname{Ram}_{\rho}\right)+\operatorname{ord}_{E_{i}}\left(\left\lceil\rho^{*}\left(K_{Z}-\theta^{*}\left(K_{X}+\Delta_{X}\right)\right)\right\rceil\right) \\
& =\operatorname{ord}_{E_{i}}(h)+\operatorname{ord}_{E_{i}}\left(\left\lceil K_{W}-\eta^{*}\left(K_{Y}+\Delta_{Y}\right)\right\rceil\right) .
\end{aligned}
$$

Thus, we conclude $h \in \mathcal{J}\left(B ; \Delta_{Y}\right)$ and hence $\mathcal{J}\left(A ; \Delta_{X}\right) \cdot \pi_{*} \mathscr{O}_{Y} \subseteq \pi_{*} \mathcal{J}\left(B ; \Delta_{Y}\right)$.

Now assume in addition that the trace map is surjective, i.e. $\operatorname{Tr}(B)=A$. We have, using the surjectivity of trace at the third inequality, that

$\mathcal{J}\left(A ; \Delta_{X}\right) \subseteq\left(\mathcal{J}\left(A ; \Delta_{X}\right) \cdot \pi_{*} \mathscr{O}_{Y}\right) \cap \mathscr{O}_{X} \subseteq \pi_{*} \mathcal{J}\left(B ; \Delta_{Y}\right) \cap \mathscr{O}_{X} \subseteq \operatorname{Tr}\left(\pi_{*} \mathcal{J}\left(B ; \Delta_{Y}\right)\right) \subseteq \mathcal{J}\left(A ; \Delta_{X}\right)$.

This necessitates equality throughout, which completes the proof.

We now complete the proof of our main theorem.

Corollary 8.2. Suppose that $(X, \Delta)$ is a pair in characteristic zero. Then

$$
\mathcal{J}(X ; \Delta)=\bigcap_{\pi: Y \longrightarrow X} \text { Image }\left(\pi_{*} \mathscr{O}_{Y}\left(\left\lceil K_{Y}-\pi^{*}\left(K_{X}+\Delta\right)\right\rceil\right) \stackrel{\operatorname{Tr}}{\longrightarrow} K(X)\right)
$$

where $\pi$ ranges over all alterations with $Y$ normal, $\operatorname{Tr}: K(Y) \rightarrow K(X)$ is the field trace, and we have that $K_{Y}=\pi^{*} K_{X}+\operatorname{Ram}_{\pi}$ wherever $\pi$ is finite.

Proof. Because of the existence of resolution of singularities in characteristic zero, we may assume that each $Y$ is smooth and that $K_{Y}-\pi^{*}\left(K_{X}+\Delta\right)$ is supported on a simple normal crossings divisor on $Y$. First consider a finite map $f: W \rightarrow X$ with $W$ normal (note that we are in characteristic zero, so the map is separable). If we pick a representative $K_{X}$ such that $\mathscr{O}_{X}\left(K_{X}\right)=\omega_{X}$ and also pick $K_{W}=f^{*} K_{X}+\operatorname{Ram}_{f}$, then we recall from Example 2.2 that the field trace $\operatorname{Tr}: K(W) \rightarrow K(X)$ induces a map $\operatorname{Tr}: \mathscr{O}_{W}\left(K_{W}\right) \rightarrow \mathscr{O}_{X}\left(K_{X}\right)$ which is identified with the Grothendieck trace $\operatorname{Tr}_{f}: f_{*} \omega_{W} \rightarrow \omega_{X}$.

Fix any proper dominant map $\pi: Y \rightarrow X$. Factor $\pi$ through a birational map $\rho: Y \rightarrow W$ and a finite map $f: W \rightarrow X$ with $W$ normal via Stein factorization. Thus $\pi_{*} \omega_{Y} \rightarrow \omega_{X}$ factors through $\operatorname{Tr}: f_{*} \omega_{W} \rightarrow \omega_{X}$. It follows that

$$
\pi_{*} \mathscr{O}_{Y}\left(K_{Y}-\pi^{*}\left(K_{X}+\Delta\right)\right) \rightarrow K(X)
$$

factors through $\operatorname{Tr}: K(W) \rightarrow K(X)$. Furthermore, $\rho_{*} \mathscr{O}_{Y}\left(K_{Y}-\pi^{*}\left(K_{X}+\Delta\right)\right)$ is by definition the multiplier ideal $\mathcal{J}\left(W ; \Delta_{W}\right)$ where $\Delta_{W}=f^{*} \Delta-\operatorname{Ram}_{f}$, since $Y$ is smooth. Thus, 
Image $\left(\pi_{*} \mathscr{O}_{Y}\left(K_{Y}-\pi^{*}\left(K_{X}+\Delta\right)\right) \rightarrow K(X)\right)$ is simply $\operatorname{Tr}\left(\rho_{*} \mathcal{J}\left(W ; \Delta_{W}\right)\right)$. But that is equal to $\mathcal{J}(X ; \Delta)$ by Theorem 8.1 .

Finally, we turn our attention to the behavior of the multiplier ideal under proper dominant maps in characteristic zero.

Theorem 8.3. If $(X, \Delta)$ is a log- $\mathbb{Q}$-Gorenstein pair in characteristic zero, then

$$
\mathcal{J}(X ; \Delta)=\bigcap_{\pi: Y \rightarrow X} \operatorname{Image}\left(\mathbf{h}^{\operatorname{dim} Y-\operatorname{dim} X} \mathbf{R} \pi_{*} \mathscr{O}_{Y}\left(\left\lceil K_{Y}-\pi^{*}\left(K_{X}+\Delta\right)\right\rceil\right) \stackrel{\operatorname{Tr}_{\pi}}{\longrightarrow} K(X)\right)
$$

where the intersection runs over all proper dominant maps from normal varieties $Y$. Note the map to $K(X)$ is induced from the trace map as in Proposition 2.18.

Proof. We may restrict our maps $\pi: Y \rightarrow X$ to those maps where $Y$ is regular and which factor through a fixed regular alteration $f: Z \rightarrow X$ such that $f^{*}\left(K_{X}+\Delta\right)$ is an integral Cartier divisor. Thus by Corollary 8.2, it is sufficient to show that

$$
\mathbf{h}^{\operatorname{dim} Y-\operatorname{dim} X} \mathbf{R}(f \circ \rho)_{*} \mathscr{O}_{Y}\left(K_{Y}-\pi^{*}\left(K_{X}+\Delta\right)\right) \rightarrow \mathbf{h}^{0} \mathbf{R} f_{*} \omega_{Z}\left(-f^{*}\left(K_{X}+\Delta\right)\right)
$$

is surjective on global sections. However, by the statement of [Kov00, Theorem 2], first correctly proved in full generality in [Bha10, Theorem 4.1.3], the natural map $\mathscr{O}_{Z} \rightarrow \mathbf{R} \rho_{*} \mathscr{O}_{Y}$ has a left inverse in the derived category, and thus so does

$$
\mathbf{R} f_{*} \mathbf{R} \rho_{*}\left(\omega_{Y}[\operatorname{dim} Y] \otimes \mathscr{O}_{Y}\left(\pi^{*}\left(K_{X}+\Delta\right)\right)\right) \rightarrow \mathbf{R} f_{*}\left(\omega_{Z}[\operatorname{dim} Z] \otimes \mathscr{O}_{Z}\left(f^{*}\left(K_{X}+\Delta\right)\right)\right) .
$$

In particular, taking $-d$ th cohomology yields the desired surjection on global sections.

\section{Further QUESTIONS}

This theory suggests a large number of potential directions for further inquiry. We highlight a few of the more obvious ones below.

Question 9.1 (Mixed characteristic). What can be said in mixed characteristic? In particular, does the intersection from our Main Theorem stabilize for schemes in mixed characteristic?

We have learned that M. Hochster and W. Zhang have made progress on this question in low dimensions for isolated singularities.

Question 9.2 (Adjoint ideals). Can one develop a characteristic theory analogous to the theory of adjoint ideals, $c f$. [Laz04, 9.3.E] or [Tak08, described via alterations or finite covers?

The characterization of test ideals, as well as $F$-regular and $F$-rational singularities suggests the following:

Question 9.3 ( $F$-pure singularities). Can $F$-pure (or $F$-injective) singularities likewise be described by alterations?

Finally, we consider the following question:

Question 9.4 (Effectivity of covers and alterations). Given a pair $(X, \Delta)$, how can we identify finite covers (or alterations) $\pi: Y \rightarrow X$ such that

$$
\tau(X ; \Delta)=\text { Image }\left(\left\lceil\pi_{*} \mathscr{O}_{Y}\left(K_{Y}-\pi^{*}\left(K_{X}+\Delta\right)\right\rceil\right) \stackrel{\operatorname{Tr}_{\pi}}{\longrightarrow} K(X)\right) ?
$$

In other words, can we determine when the intersection from our Main Theorem stabilizes? 
We do not have a good answer to this question. The key point in our construction is repeated use of the Equational Lemma HH92, HL07, SS12. The procedure in that Lemma is constructive. However, this is not very satisfying. It would be very satisfying and likely useful if one had a different geometric or homological criterion for identifying $\pi: Y \rightarrow X$ as in the question above.

\section{REFERENCES}

[AJ89] D. Arapura And D. B. Jaffe: On Kodaira vanishing for singular varieties, Proc. Amer. Math. Soc. 105 (1989), no. 4, 911-916. MR952313 (89h:14013)

[Bha10] B. Bhatт: Derived direct summands, ProQuest LLC, Ann Arbor, MI, 2010, Thesis (Ph.D.)Princeton University. 2753219

[Bha12] B. BнAтт: Derived splinters in positive characteristic, Compos. Math. 148 (2012), no. 6, 1757-1786. 2999303

[Bli04] M. Blickle: Multiplier ideals and modules on toric varieties, Math. Z. 248 (2004), no. 1, 113-121. MR2092724 (2006a:14082)

[Bli13] M. Blickle: Test ideals via algebras of $p^{-e}$-linear maps, J. Algebraic Geom. 22 (2013), no. 1, 49-83. 2993047

[BB11] M. Blickle And G. Böckle: Cartier modules: finiteness results, J. Reine Angew. Math. 661 (2011), 85-123. 2863904

[BSTZ10] M. Blickle, K. Schwede, S. Takagi, And W. Zhang: Discreteness and rationality of F-jumping numbers on singular varieties, Math. Ann. 347 (2010), no. 4, 917-949. 2658149

[Bou98] N. Bourbaki: Commutative algebra. Chapters 1-7, Elements of Mathematics (Berlin), Springer-Verlag, Berlin, 1998, Translated from the French, Reprint of the 1989 English translation. MR1727221 (2001g:13001)

[CR11] A. Chatzistamatiou And K. Rülling: Higher direct images of the structure sheaf in positive characteristic, Algebra Number Theory 5 (2011), no. 6, 693-775. 2923726

[Con00] B. ConRAD: Grothendieck duality and base change, Lecture Notes in Mathematics, vol. 1750, Springer-Verlag, Berlin, 2000. MR1804902 (2002d:14025)

[dJ96] A. J. DE Jong: Smoothness, semi-stability and alterations, Inst. Hautes Études Sci. Publ. Math. (1996), no. 83, 51-93. 1423020 (98e:14011)

[Ein97] L. EIN: Multiplier ideals, vanishing theorems and applications, Algebraic geometry-Santa Cruz 1995, Proc. Sympos. Pure Math., vol. 62, Amer. Math. Soc., Providence, RI, 1997, pp. 203-219. MR1492524 (98m:14006)

[FW89] R. FEDder AND K. WATANABe: A characterization of F-regularity in terms of F-purity, Commutative algebra (Berkeley, CA, 1987), Math. Sci. Res. Inst. Publ., vol. 15, Springer, New York, 1989, pp. 227-245. MR1015520 (91k:13009)

[GR70] H. GRAuert AND O. RiEmenschneider: Verschwindungssätze für analytische Kohomologiegruppen auf komplexen Räumen, Invent. Math. 11 (1970), 263-292. MR0302938 (46 \#2081)

[Har66] R. HARTSHorne: Residues and duality, Lecture notes of a seminar on the work of A. Grothendieck, given at Harvard 1963/64. With an appendix by P. Deligne. Lecture Notes in Mathematics, No. 20, Springer-Verlag, Berlin, 1966. MR0222093 (36 \#5145)

[Har77] R. Hartshorne: Algebraic geometry, Springer-Verlag, New York, 1977, Graduate Texts in Mathematics, No. 52. MR0463157 (57 \#3116)

[Har07] R. HARtshorne: Generalized divisors and biliaison, Illinois J. Math. 51 (2007), no. 1, 83-98 (electronic). MR2346188

[Hir64] H. HiRONAKA: Resolution of singularities of an algebraic variety over a field of characteristic zero. I, II, Ann. of Math. (2) 79 (1964), 109-203; ibid. (2) 79 (1964), 205-326. MR0199184 (33 \#7333)

[HY11] M. Hochster AND Y. YAO: A weak embedding theorem (and test exponents) for modules of finite phantom projective dimension, work in progress, 2011.

[HH92] M. Hochster And C. Huneke: Infinite integral extensions and big Cohen-Macaulay algebras, Ann. of Math. (2) 135 (1992), no. 1, 53-89. 1147957 (92m:13023)

[HL07] C. Huneke And G. Lyubeznik: Absolute integral closure in positive characteristic, Adv. Math. 210 (2007), no. 2, 498-504. 2303230 (2008d:13005)

[Kaw82] Y. KawAmata: A generalization of Kodaira-Ramanujam's vanishing theorem, Math. Ann. 261 (1982), no. 1, 43-46. MR675204 (84i:14022) 
[KKMSD73] G. Kempf, F. F. Knudsen, D. Mumford, And B. Saint-Donat: Toroidal embeddings. I, Lecture Notes in Mathematics, Vol. 339, Springer-Verlag, Berlin, 1973. MR0335518 (49 \#299)

[KM98] J. Kollár AND S. Mori: Birational geometry of algebraic varieties, Cambridge Tracts in Mathematics, vol. 134, Cambridge University Press, Cambridge, 1998, With the collaboration of C. H. Clemens and A. Corti, Translated from the 1998 Japanese original. MR1658959 (2000b:14018)

[Kov00] S. J. KovÁcs: A characterization of rational singularities, Duke Math. J. 102 (2000), no. 2, 187-191. MR1749436 (2002b:14005)

[Laz04] R. LAzARsfeld: Positivity in algebraic geometry. II, Ergebnisse der Mathematik und ihrer Grenzgebiete. 3. Folge. A Series of Modern Surveys in Mathematics [Results in Mathematics and Related Areas. 3rd Series. A Series of Modern Surveys in Mathematics], vol. 49, Springer-Verlag, Berlin, 2004, Positivity for vector bundles, and multiplier ideals. MR2095472 (2005k:14001b)

[Lip78] J. Lipman: Desingularization of two-dimensional schemes, Ann. Math. (2) 107 (1978), no. 1, 151-207. 0491722 (58 \#10924)

[Lip94] J. LiPMAN: Adjoints of ideals in regular local rings, Math. Res. Lett. 1 (1994), no. 6, 739-755, With an appendix by Steven Dale Cutkosky. MR1306018 (95k:13028)

[LT81] J. Lipman And B. Teissier: Pseudorational local rings and a theorem of Briançon-Skoda about integral closures of ideals, Michigan Math. J. 28 (1981), no. 1, 97-116. MR600418 (82f:14004)

[Ray78] M. RAYNAud: Contre-exemple au "vanishing theorem" en caractéristique $p>0$, C. P. Ramanujam - a tribute, Tata Inst. Fund. Res. Studies in Math., vol. 8, Springer, Berlin, 1978, pp. 273-278. 541027 (81b:14011)

[SS12] A. SAnnai And A. K. Singh: Galois extensions, plus closure, and maps on local cohomology, Adv. Math. 229 (2012), no. 3, 1847-1861.

[Sch09] K. Schwede: F-adjunction, Algebra Number Theory 3 (2009), no. 8, 907-950.

[Sch10] K. Schwede: Centers of F-purity, Math. Z. 265 (2010), no. 3, 687-714. 2644316

[Sch11] K. SchwedE: Test ideals in non-QQQ-Gorenstein rings, Trans. Amer. Math. Soc. 363 (2011), no. 11, 5925-5941. 2817415 (2012c:13011)

[ST08] K. Schwede And S. TAKagi: Rational singularities associated to pairs, Michigan Math. J. 57 (2008), 625-658.

[ST10] K. Schwede AND K. TUCKER: On the behavior of test ideals under finite morphisms, arXiv:1003.4333, to appear in J. Algebraic Geom.

[ST12a] K. Schwede And K. Tucker: A survey of test ideals, Progress in commutative algebra 2, Walter de Gruyter, Berlin, 2012, pp. 39-99. 2932591

[ST12b] K. Schwede AND K. TuCKer: Test ideals of non-principal ideals: Computations, jumping numbers, alterations and division theorems, arXiv:1212.6956, to appear in Journal de Mathématiques Pures et Appliquées.

[Sin99] A. K. SingH: Q-Gorenstein splinter rings of characteristic p are F-regular, Math. Proc. Cambridge Philos. Soc. 127 (1999), no. 2, 201-205. 1735920 (2000j:13006)

[Smi94] K. E. Sмiтн: Tight closure of parameter ideals, Invent. Math. 115 (1994), no. 1, 41-60. MR1248078 (94k:13006)

[Smi95] K. E. Smith: Test ideals in local rings, Trans. Amer. Math. Soc. 347 (1995), no. 9, 3453-3472. MR1311917 (96c:13008)

[Smi97a] K. E. Smith: Erratun to vanishing, singularities and effective bounds via prime characteristic local algebra, http://www.math.1sa.umich.edu/ kesmith/santaerratum.ps, 1997.

[Smi97b] K. E. Sмith: F-rational rings have rational singularities, Amer. J. Math. 119 (1997), no. 1, 159-180. MR1428062 (97k:13004)

[Smi97c] K. E. Smith: Vanishing, singularities and effective bounds via prime characteristic local algebra, Algebraic geometry - Santa Cruz 1995, Proc. Sympos. Pure Math., vol. 62, Amer. Math. Soc., Providence, RI, 1997, pp. 289-325. MR1492526 (99a:14026)

[Tak04] S. TAKAGI: An interpretation of multiplier ideals via tight closure, J. Algebraic Geom. 13 (2004), no. 2, 393-415. MR2047704 (2005c:13002)

[Tak08] S. TAKAgi: A characteristic $p$ analogue of plt singularities and adjoint ideals, Math. Z. 259 (2008), no. 2, 321-341. MR2390084 (2009b:13004)

[TW92] M. TOMARI AND K. WATAnABE: Normal $Z_{r}$-graded rings and normal cyclic covers, Manuscripta Math. 76 (1992), no. 3-4, 325-340. 1185023 (93j:13002) 
[Vie82] E. Vienweg: Vanishing theorems, J. Reine Angew. Math. 335 (1982), 1-8. MR667459 (83m:14011)

Institut für Mathematik, Johannes Gutenberg-Universität Mainz, 55099 Mainz, Germany

E-mail address: blicklem@uni-mainz.de

Department of Mathematics, The Pennsylvania State University, University Park, PA, 16802, USA

E-mail address: schwede@math.psu.edu

Department of Mathematics, University of Utah, Salt Lake City, UT, 84112, USA

E-mail address: kevtuck@math.utah.edu 